\title{
34. GEOTECHNICAL PROPERTIES OF NORTHWEST PACIFIC PELAGIC CLAYS: DEEP SEA DRILLING PROJECT LEG 86, HOLE 576A ${ }^{1}$
}

\author{
The Marine Geotechnical Consortium²
}

\begin{abstract}
Geotechnical property results from Deep Sea Drilling Project Hole 576A sediments represent the most complete data set ever compiled for a continuously depositional, sedimentary section that spans $65 \mathrm{~m} . \mathrm{y}$. Geotechnical analyses included index-property and sediment characterization, and consolidation, permeability, and triaxial testing. Excellent agreement exists between test data generated in this multilaboratory effort.

Sediment characterization and index-property data delineate three well-defined units that conform with major lithologic divisions. Within Subunit IA these data appear inconsistent with accepted geotechnical relationships, showing water content, plasticity, and grain size increasing over similar intervals. These unusual relationships are attributed, in part, to silt-sized bonded clay aggregates. Within Subunit IB geotechnical properties become more uniform, and a marked increase in X-ray amorphous material occurs below $28 \mathrm{~m}$ sub-bottom. A pronounced change in the geotechnical behavior of Hole 576A sediments occurs in Unit II and corresponds to the presence of calcareous oozes.

Consolidation test results show overconsolidated sediments in the upper $28 \mathrm{~m}$ and normally consolidated sediments between 28 and $55 \mathrm{~m}$ sub-bottom. Mechanisms to explain the state of overconsolidation that are consistent with the geological history of the area include cementation and interparticle bonding, the release of high hydrostatic pressures during core recovery, and artifacts resulting from the consolidation test process. Additionally, the relevance of the preconsolidation stress determined using consolidation test results may be limited.

Variations in the shear strength gradient with depth are consistent with the consolidation test results. The gradient for the overconsolidated interval ( 0 and $28 \mathrm{~m}$ sub-bottom) is significantly higher than between 28 and $55 \mathrm{~m}$ sub-bottom, where the sediments become normally consolidated.
\end{abstract}

\section{INTRODUCTION}

The constraints of standard coring techniques have limited most studies of the geotechnical properties of marine sediments to samples recovered from less than $15 \mathrm{~m}$ sub-bottom. Many of these studies have tried to relate variations in geotechnical properties to variations in geological processes. In many instances, however, the relative geological complexity of the area or an insufficient geotechnical data base have precluded the development of such relationships. At Deep Sea Drilling Project (DSDP) Site 576, we were able to evaluate these relationships for a fairly simple, continuously depositional, pelagic clay environment whose geological history is well substantiated over the last 65 m.y.

Site 576 was triple cored (Holes 576, 576A, 576B) to get enough sediment samples for detailed sedimentological, stratigraphic, geochemical, and geotechnical analyses. At the request of the Marine Geological Consortium, Hole 576A was continuously cored and preserved in the full-round for detailed shore-based geotechnical property analyses. These analyses were intended to evaluate

\footnotetext{
${ }^{1}$ Heath, G. R., Burckle, L. H., et al., Init. Repts. DSDP, 86: Washington (U.S. Govt.

Printing Office).
2 The Marine Geotechnical Consortium for Deep Sea Drilling Project Leg 86 consisted of the University of Colorado (CU), Boulder, Colorado; Humboldt State University (HSU), Arcata, Cailfornia; Institute of Oceanographic Sciences (IOS), Wormley, Godalming, United Kingdom; Oregon State University (OSU), Corvallis, Oregon; San Diego State University (SDSU), San Diego, California; Sandia National Laboratories (SNL), Albuquerque, New Mexico; Texas A\&M University (TAMU), College Station, Texas; University of Rhode island (UR1), Narragansett, Rhode Island; and United States Geological Survey (USGS). Denver, Colorado. The following persons contributed to this study. CU: Robert Schiffman, Andrew Dean: HSU: Ronald Chaney, J. Suehanek, B. Vega. T. McFarland; IOS: Peter J. Schultheiss, David Gunn; OSU: George Keller; SDSU: Iraj Noorany, M. Faghihi; SNL: Les Shephard, Joel Lipkin, Jean Kinker; TAMU: William Bryant, Elliot Taylor, Mark Johns; URI: Armand Silva, William Levy, Ricci Siciliano, Kathleen Dadey; USGS: Harold Olsen.
}

the interrelationships between the geotechnical properties and the geological processes of an abyssal hill environment, specifically as they pertain to variations in the sediment stress history, consolidation-permeability relationships, and shear strength.

\section{GEOLOGIC SETTING}

Site 576 is located east of Shatsky Rise in the western North Pacific red clay province (Fig. 1) very near magnetic Anomaly M13, which indicates a basement age of 129 m.y. (Site 576 chapter, this volume). Regional seafloor morphology and near-surface seismic profile characteristics have been mapped and discussed by Damuth et al. (1983). The location of Site 576 is characterized by a smooth to gently rolling seafloor. Seafloor bathymetry gradually deepens from southwest to northeast at a gradient of approximately 1:1000, reflecting an abyssal hill oceanic basement covered by pelagic sediments. Water depth at Site 576 is $6127 \mathrm{~m}$.

The sedimentary section of Hole 576A can be divided into three general lithologic units on the basis of macroscopic descriptions and smear-slide analyses (Site 576 chapter, this volume; Fig. 2). Unit I consists of pelagic, brown clay extending to $55 \mathrm{~m}$ sub-bottom that can be divided into two subunits. Subunit IA (0 to $28 \mathrm{~m}$ sub-bottom) is a pelagic, yellowish brown, burrowed clay. The abundance of burrows and the silt-sized fraction generally decrease down through Subunit IA. Subunit IB (28 to $55 \mathrm{~m}$ sub-bottom) is an unburrowed, homogeneous, dark brown, pelagic clay generally containing less than $20 \%$ silt. The contact between these subunits is gradational over several meters. Unit II is composed of interbedded carbonate oozes and brown pelagic clays ( 55 to 


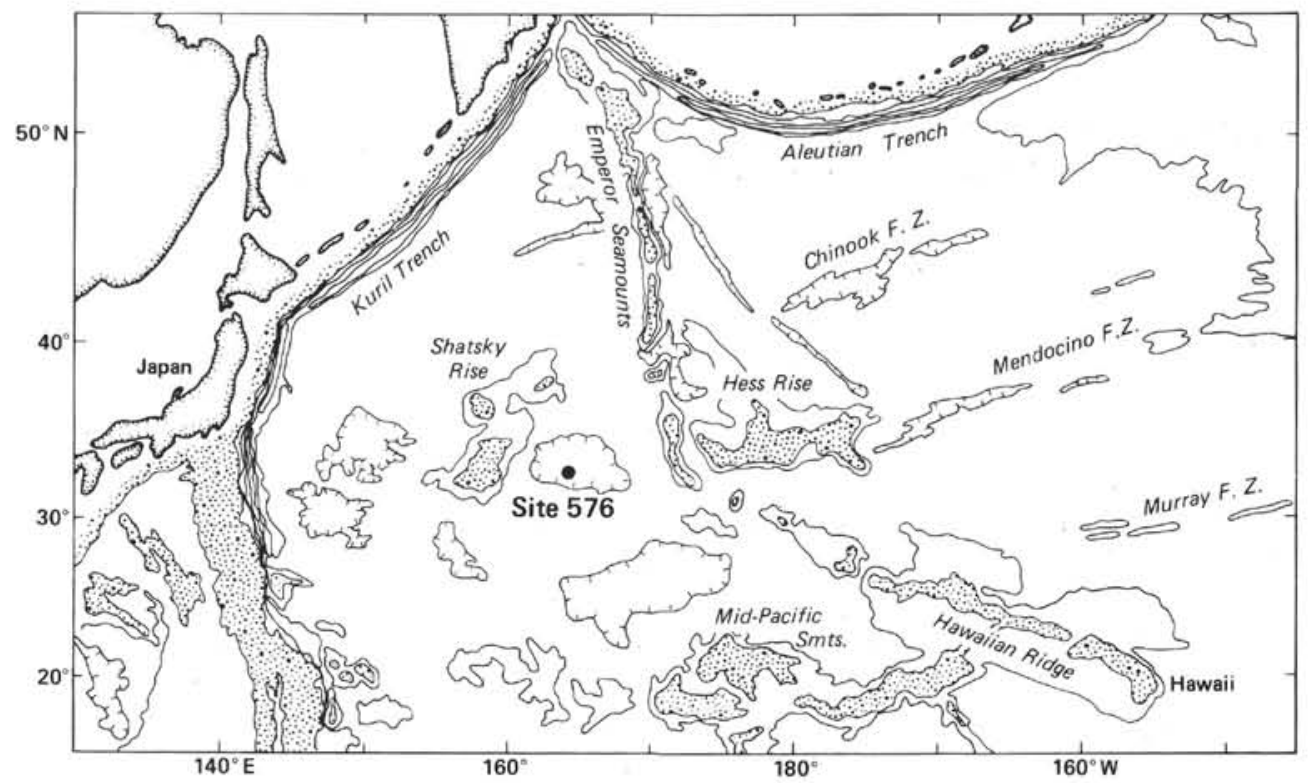

Figure 1. Location of Site 576 in the northwest Pacific. Areas shallower than $4 \mathrm{~km}$ stippled, 5-km contour plain, 6-km contour hachured.

$66 \mathrm{~m}$ sub-bottom). Evidence of graded bedding and crossbedding in some of the carbonate ooze layers and sharp basal contacts between the carbonates and interbedded pelagic clays suggest a turbidite origin for at least some of the oozes. The base of Unit II was penetrated only in Hole 576B, at a sub-bottom depth of $76 \mathrm{~m}$. Unit III, recovered only at the base of i Iole 576B, consists of a chert or silicified clay unit.

Sedimentation rates (uncorrected for consolidation) decrease downcore in Unit I from $10 \mathrm{~m} / \mathrm{m}$.y. at the surface to less than $3 \mathrm{~m} / \mathrm{m}$.y. at the base of the Matuyama Epoch ( 20 m sub-bottom; Heath, Rea, and Levi, this volume). This downcore decrease in sedimentation rate reflects the change from primarily eolian material in Subunit IA to an increased authigenic sediment component in Subunit IB. The change in dominant sediment components results from changes in depositional patterns as the location of Site 576 moved north and west in and out of various wind belts in response to seafloor spreading (Leinen, this volume).

\section{GEOTECHNICAL CORE PROCESSING}

Hole 576A cores were obtained by the Glomar Challenger on Leg 86 using the hydraulic piston corer. Immediately after recovery each full-round core was cut into 1.5 -m-long sections, capped, taped, annotated, and stored upright at $4^{\circ} \mathrm{C}$ in the ship's cold room. Upon arrival in Japan the cores were air-freighted to the DSDP repository at Scripps Institution of Oceanography (SIO), where they were again stored upright at $4^{\circ} \mathrm{C}$ prior to geotechnical sampling in September 1982.

Members of the consortium institutions and laboratories were present for all geotechnical sampling. Prior to sub-sampling, each core section was examined visually through the core liner to identify areas of obvious disturbance and to note major changes in lithology. These lithologic changes were correlated with shipboard core

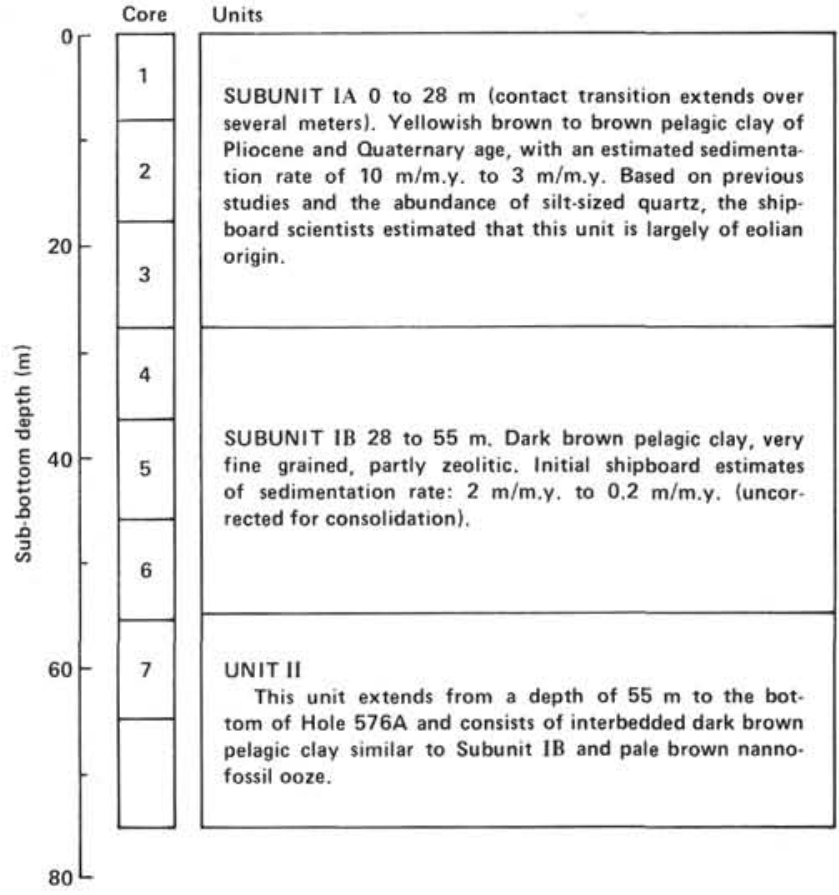

Figure 2. Generalized description of the lithologic section recovered at Hole 576A.

descriptions for Holes 576 and 576B to develop a coreprocessing plan that would adequately sample each major lithologic unit.

The core-processing procedure involved cutting the 1.5-m full-round sections into shorter, full-round sections (15 to $40 \mathrm{~cm}$ ) that were capped, taped, and waxed for subsequent detailed geotechnical analyses. Consortium members hand-carried these sections from SIO to their respective laboratories, where they were radiographed, described, and stored in a cold room prior to being tested. 
Analyses performed on the ends of the shorter, fullround sections before capping and sealing at SIO included visual core descriptions and measurements of vane shear strength, water content, bulk density, and compressional velocity. Compressional-velocity measurements were performed parallel to bedding; the vane shear shaft was oriented normal to bedding. In addition, clay mineralogy and fabric samples were obtained from the core section ends for laboratory analyses. All full-round core sections not selected for laboratory analyses at that time were cut into $25-\mathrm{cm}$ full-round sections and stored vertically in the DSDP repository cold room for possible future sample requests.

Additional core processing and sampling were performed by the consortium in June 1983. At that time, a number of remaining full-round sections were distributed to interested consortium laboratories before longitudinally splitting and describing the remaining full-round sections. Geotechnical measurements were performed on the split sections in the manner described above, except that in the vane shear measurements the vane shaft was oriented parallel to bedding.

\section{TEST PROCEDURES AND METHODS}

\section{Sediment Characterization and Index Properties}

Table 1 summarizes the tests performed during this study. For most of the standard geotechnical tests (e.g., index properties, sediment classification), the appropriate American Society for Testing and Materials (ASTM) Annual Book of Standards is referenced (Table 1). For nonstandardized tests (e.g., consolidation, triaxial tests), suitable references in the geotechnical literature are cited. Variations from standard procedures (e.g., salt corrections) are also noted. Lambe (1951) defines the standard geotechnical terminology (e.g., plastic limit, activity) used throughout this chapter.

All samples hand-carried to the individual laboratories were X-rayed prior to subsampling to assess the degree of core disturbance or flow-in. If any visible signs of disturbance or flow-in were observed, the sample was not tested. Tabulated results include data obtained during both sample processing sessions at SIO and further analyses at the individual consortium laboratories.

\section{Consolidation and Permeability Tests}

One-dimensional consolidation tests were performed using three different laboratory procedures. The standard consolidation test involves the incremental loading of a relatively thin, laterally confined sediment sample. Axial strain-time relationships are obtained by measuring the change in sample height during the test. A complete description of this technique can be found in Lambe (1951). A variation of this method involves the application of sufficient back pressure to redissolve gas bubbles and completely saturate the consolidation sample. Lowe et al. (1964) describe the benefits of this method. A serious drawback to both of the above methods is that more than 15 days are required to complete a test. Another test used was the constant rate of deformation (CRD) test, which only requires a few hours per test. The CRD test imposes a uniaxial deformation at a prescribed constant rate and provides a continuous record of the changes of consolidation parameters with respect to the applied effective stress. Gorman et al. (1978), Znidarcic (1982), and Znidarcic and Schiffman (1983) describe the CRD method in detail.

Two fundamental properties that contribute directly to the consolidation process are compressibility and permeability. Compressibility is defined as the amount of sediment volume compression per unit increment of stress. The compression index $\left(C_{\mathrm{c}}\right)$ is calculated from the slope of the void ratio-effective stress ( $e$ versus $\log \sigma^{\prime}$ ) curve obtained from consolidation test results as follows:

$$
C_{\mathrm{c}}=\frac{-\Delta e}{\left(\Delta \log \sigma^{\prime}\right)}
$$

where $\Delta e$ is the void ratio change over a corresponding $\log$ increment of the effective stress $\left(\sigma^{\prime}\right)$ (Lambe, 1951). The compression index is reserved for a loading sequence. If an unloading sequence is being analyzed, the companion term is the expansion index, $C_{\mathrm{e}}$.

Permeability is defined as the capacity of a sediment to transmit fluid and is calculated by the ratio of the volumetric rate of flow to the gradient that drives fluid through a cross-sectional area of sediment. Three methods available to measure permeability directly include: (1) constant head, (2) falling head, and (3) flow pump. The constant-head method measures the flow rate under an applied pressure gradient. The falling-head method, which is the conventional method used for fine-grained sediments, relates the flow rate to a constantly changing hydraulic gradient. These conventional tests are described in Lambe (1951). The flow-pump method measures the pressure gradient while maintaining a controlled flow rate (Olsen, 1966). A critical assessment of each of these methods (Pane et al., 1983) has shown that permeability results for soft clays may be biased by seepage-induced consolidation unless very small pressure gradients are used.

In addition to measuring permeability directly, two indirect methods were used to deduce the coefficient of permeability from the theoretical consolidation relationships (Znidarcic and Schiffman, 1983). The first method uses the results from the standard consolidation test and is based on linear, infinitesimal strain consolidation theory. The second method uses CRD test results and nonlinear, finite strain, consolidation theory to calculate the coefficient of permeability.

\section{Strength Tests}

Several different types of strength tests were performed on Hole 576A sediments. In addition to the laboratory vane shear tests performed during the core processing, three other types of strength tests were completed: (1) unconsolidated-undrained triaxial compression (UU); (2) isotropically consolidated, undrained triaxial compression (CIU); and (3) anistropically consolidated-undrained triaxial compression (CAU; Table 1). Samples for these tests were prepared by cutting a length of core (typically $100 \mathrm{~mm}$ ) contained in a plastic core-liner tube 
Table 1. Summary of referenced procedures used in the geotechnical property analysis of Hole 576A sediments.

\begin{tabular}{|c|c|c|}
\hline Test & $\begin{array}{l}\text { Reference for } \\
\text { test procedure }\end{array}$ & $\begin{array}{l}\text { Variations from } \\
\text { standard procedure }\end{array}$ \\
\hline \multicolumn{3}{|l|}{$\begin{array}{l}\text { Sediment characterization } \\
\text { Index properties }\end{array}$} \\
\hline Clay mineralogy & Heath and Pisias (1979) & Talc internal standard \\
\hline Water content & ASTM D2216 & Salt corrected, Noorany (1984) \\
\hline Liquid limit & ASTM D423 & Salt corrected, Noorany (1984) \\
\hline Plastic limit & ASTM D424 & Salt corrected, Noorany (1984) \\
\hline Specific gravity & ASTM D854 & $\begin{array}{l}\text { Some tests done using air-compari- } \\
\text { son pycnometers }\end{array}$ \\
\hline Wet-bulk density & ASTM D2937 & $\begin{array}{l}\text { Small rings (1-in. diam. and less) } \\
\text { and piston subsamplers used in } \\
\text { core }\end{array}$ \\
\hline Grain size (pipette method) & Folk (1974) & Desalinated \\
\hline Compressional wave velocity & Hamilton (1965) & $\begin{array}{l}\text { Measurements made directly in the } \\
\text { sediment, not through the core } \\
\text { liner }\end{array}$ \\
\hline \multicolumn{3}{|l|}{ Consolidation } \\
\hline Consolidation (standard) & ASTM D2435 & $\begin{array}{l}\text { Load increment ratio of } 0.5 \text { used in } \\
\text { some tests }\end{array}$ \\
\hline Consolidation (back-pressured) & Lowe et al. (1964) & \\
\hline Consolidation (constant rate of strain) & Znidarcic and Schiffman (1983) & \\
\hline \multicolumn{3}{|l|}{ Permeability } \\
\hline Constant head & Olsen (1966) & \\
\hline Falling head & Silva et al. (1981) & \\
\hline Flow pump & Pane et al. (1983) & \\
\hline \multicolumn{3}{|l|}{ Strength Tests } \\
\hline Vane shear & & $\begin{array}{l}\text { Rotation rate }=60^{\circ} / \mathrm{min} . \\
\text { Vane diameter and height } 12.7 \mathrm{~mm}\end{array}$ \\
\hline $\begin{array}{l}\text { Uncons nlidated-undrained } \\
\text { Consolidated-undrained }\end{array}$ & ASTM D2850 & \\
\hline $\begin{array}{l}\text { isotropically } \\
\text { anistropically }\left(\mathrm{K}_{\mathrm{o}}\right)\end{array}$ & Bishop and Henkel (1962) & \\
\hline
\end{tabular}

Note: Variations from the referenced standard procedures are also noted in the text.

with either a hand saw or a high-speed saw. After cutting, the sample was extruded directly from the core liner or the liner was split longitudinally and a wire saw was then used to separate and remove the sample from the core-liner halves. The sample was then trimmed to a final diameter of $35 \mathrm{~mm}$ and a height of 70 to $85 \mathrm{~mm}$ using a vertical sample lathe and a wire saw.

\section{SEDIMENT CHARACTERIZATION AND INDEX PROPERTIES}

\section{Clay Mineralogy}

Quantitative clay mineralogy results on sediments obtained with piston cores close to Site 576 are consistent with known clay mineral distribution patterns in the North Pacific (Leinen and King, in press; Heath and Pisias, 1979; Griffin et al., 1968). Leinen and King reported data from samples located at the Brunhes/Matuyama boundary (ranging in sub-bottom depth from 3.84 to $5.92 \mathrm{~m}$ ) within a $10^{4}-\mathrm{km}^{2}$ area around Site 576 and found virtually no variation in the $<20 \mu \mathrm{m}$ opal-free mineral assemblage. Illite is the dominant clay mineral present $(\sim 39 \%)$, with lesser amounts of chlorite $(\sim 19 \%)$ and minor amounts of kaolinite and smectite $(\sim 10 \%)$. Detailed downcore clay mineral analysis on a piston core located approximately $10 \mathrm{~km}$ from Site 576 shows a slight increase in smectite (5-6\% to $12-15 \%)$ and decrease in chlorite $(\sim 38 \%$ to $20-25 \%)$ with depth to $12 \mathrm{~m}$ subbottom. Leinen and King (1985) indicate that these subtle changes suggest either a change in the weathering regime in the continental source areas for the eolian material or a change in the dominant wind patterns that transport the mineral aerosol to the northwest Pacific.

Preliminary clay mineral analyses on Hole 576A sediments have emphasized sediments in Subunit IB below $30 \mathrm{~m}$ (Table 2, M. Leinen, pers. comm., 1984). Results from one near-surface sample obtained within Subunit IA are very similar to the piston core mineralogical results summarized above, except for a slightly higher illite component (50\% as opposed to $39 \%)$ and a lower kaolinite component (trace amounts as opposed to $\sim 10 \%$ ). Using a talc internal standard, clay mineral results from Subunit IB indicate the single most important factor influencing the mineralogy is the pronounced downhole increase in the ratio of amorphous to crystalline material. This increase is particularly evident below $45 \mathrm{~m}$ sub-bottom (Table 2) and is attributed to a probable increase in the amount of iron oxides, manganese oxides, and very fine-grained material with insufficient crystal size to produce good diffractions. Another possible cause contributing to this marked increase in X-ray amorphous material may be related to the effects of grain size on the 
Table 2. Preliminary mineralogy results from Hole 576A sediment. ${ }^{\mathrm{a}}$

\begin{tabular}{ccccccccccc}
\hline $\begin{array}{c}\text { Core- } \\
\text { Section }\end{array}$ & $\begin{array}{c}\text { Sub-bottom } \\
\text { depth } \\
(\mathrm{m})\end{array}$ & $\begin{array}{c}\text { Smectite } \\
(\%)\end{array}$ & $\begin{array}{c}\text { Illite } \\
(\%)\end{array}$ & $\begin{array}{c}\text { Kaolinite } \\
(\%)\end{array}$ & $\begin{array}{c}\text { Chlorite } \\
(\%)\end{array}$ & $\begin{array}{c}\text { Plagioclase } \\
(\%)\end{array}$ & $\begin{array}{c}\text { Quartz } \\
(\%)\end{array}$ & $\begin{array}{l}\text { Total } \\
(\%)\end{array}$ & $\begin{array}{c}\text { Percent } \\
\text { amorphous }\end{array}$ & $\begin{array}{c}\text { Smectite:illite } \\
\text { ratio }\end{array}$ \\
\hline $1-1$ & 0.23 & 8 & 50 & 1 & 22 & 3 & 17 & 100 & 0 & 0.16 \\
$4-2$ & 30.25 & 9 & 23 & 5 & 6 & 3 & 9 & 55 & 45 & 0.39 \\
$4-5$ & 33.91 & 13 & 31 & 6 & 6 & 1 & 4 & 61 & 39 & 0.42 \\
$4-6$ & 35.81 & 6 & 46 & 3 & 4 & 1 & 5 & 65 & 35 & 0.13 \\
$5-5$ & 44.59 & 14 & 30 & 10 & 10 & 1 & 3 & 68 & 32 & 0.47 \\
$6-1$ & 47.61 & 3 & 8 & 3 & 2 & 1 & 4 & 26 & 74 & 0.38 \\
$6-3$ & 50.65 & 7 & 11 & 2 & 4 & 1 & 5 & 30 & 70 & 0.64 \\
$6-4$ & 53.99 & 2 & 7 & 1 & 2 & 2 & 5 & 20 & 80 & 0.29 \\
\hline
\end{tabular}

Note: Relative percentages of each component were determined using a talc internal standard and weighting factors developed for North Pacific sediments by Heath and Pisias (1979).

${ }^{\mathrm{a}} \mathrm{M}$. Leinen, unpublished observations.

standardization procedure (M. Leinen, pers. comm., 1984).

Mineralogical analyses on Hole 576A sediments used for consolidation testing (E. Taylor, pers. comm., 1984) reveal a downhole increase in the relative percentage of smectite over illite in the crystalline clay-sized fraction. However, the much larger increase in the amount of Xray amorphous material present in the clay-sized fraction (up to $80 \%$, Table 2) over the same intervals would be the controlling mineralogical factor influencing the geotechnical behavior of Hole 576A sediments.

\section{Electron Microscopy}

Scanning and transmission electron microscopy (SEM and TEM) and energy dispersion X-ray spectrometry (EDS) were performed on select Hole 576A samples to evaluate particle-to-particle relationships, orientations, and arrangements. These relationships may prove critical to enhancing our understanding of the geotechnical behavior of these sediments.

SEM micrographs within Unit I (Fig. 3) show an open, flocculated structure consisting of a series of linked clay aggregates. Intergrown clay particle domains evident as locally continuous particle arrangements are apparent in several micrographs. The origin of these intergrown domains is uncertain, although they may result from the incipient growth of authigenic clay minerals, silica compounds, or iron oxides. Indications of cementation between clay-particle contacts are evident in Figure 3D. EDS analyses on the contacts between a silt grain and other particles show an enrichment of potassium and iron that suggests cementation (Fig. 4) although, because of the limited resolution of the EDS system, no definite conclusions can be reached.

Transmission electron micrographs from Subunit IA (22.17 m sub-bottom) and Subunit IB (46.02 m sub-bottom) show a very complex clay microstructure consisting of fine-grained, high void ratio clays occurring as clay floccules or as domains oriented face-to-face (Fig. 5). Authigenic minerals and larger opaque particles are often present.

\section{Water Content}

Water contents are quite variable within all of the sediments in Subunit IA (Fig. 6 and Table 3). At the sedi- ment surface $(<50 \mathrm{~cm}$ sub-bottom) water content values range from 130 to $164 \%$. In general, water content then increases with depth to approximately $10 \mathrm{~m}$ subbottom where the values range from 156 to $223 \%$. Below $10 \mathrm{~m}$ sub-bottom both the absolute values and the range of water-content values decrease down to and through the transition zone ( $\sim 20$ to $28 \mathrm{~m}$ sub-bottom) as the sedimentary section gradually changes to Subunit IB. Values range from 102 to $142 \%$ over this interval. At $28 \mathrm{~m}$, water content is significantly higher than in the immediately overlying transition zone.

Throughout Subunit IB (28-55 m sub-bottom) water contents generally decrease (Fig. 6 ). Distinct intervals $(\sim 5 \mathrm{~m})$ of increasing and decreasing water-content values probably reflect subtle lithologic changes within Subunit IB and are not a result of variations in interlaboratory test procedures. These cyclic variations are also evident in many of the other properties discussed below.

A pronounced change in water content occurs at the contact between the overlying pelagic clay section (Unit I) and the interbedded carbonates and pelagic clays of Unit II (Fig. 6). Values at the base of Unit I (55 m subbottom) range from 110 to $120 \%$; whereas within Unit II below $55 \mathrm{~m}$, water content values decrease to near $50 \%$ within the carbonate oozes. Water content within Unit II decreases slightly with depth. The occasional high water-content values observed in Unit II are associated with the interbedded pelagic clay layers in this unit. These high values are similar to those reported at the base of Subunit IB.

\section{Atterberg Limits}

Small, local downcore variability in both liquid and plastic limit values (Fig. 7 and Table 3 ) probably results from minor procedural differences between laboratories. This variability does not, however, obscure the important downcore trends. Liquid limit exhibits a general increasing trend from $131 \%$ very near the seafloor to $186 \%$ at approximately $16 \mathrm{~m}$ sub-bottom, while the plastic limit fluctuates about an average value of $54 \%$ over the same interval. Liquid limits above $20 \mathrm{~m}$ sub-bottom are generally less than the natural water contents resulting in liquidity indices $\left(I_{\mathrm{L}}\right)$ of greater than 1.0 (Fig. 8). Below $20 \mathrm{~m}$ sub-bottom, $I_{\mathrm{L}}$ values decrease gradually throughout the remainder of Subunit IA to a minimum of ap- 

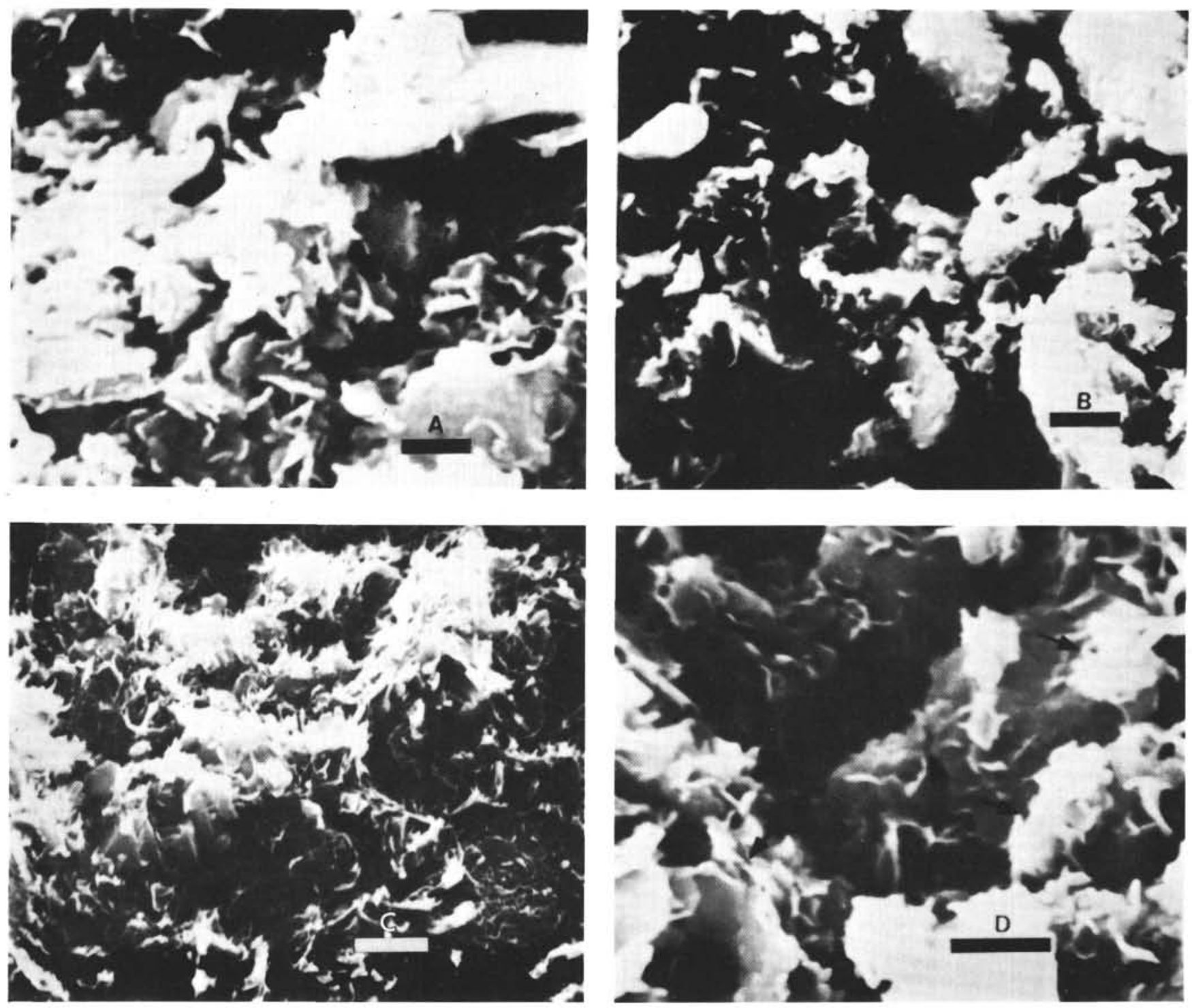

Figure 3. Scanning electron micrographs of sediments located at $11.6 \mathrm{~m}(\mathrm{~A}), 15.6 \mathrm{~m}(\mathrm{~B})$, and $43.3 \mathrm{~m}(\mathrm{C})$ sub-bottom showing intergrown clay particle domains (A, B, C), a flocculent microstructure (B), and authigenic material (C). Arrows on micrograph D (11.6 m sub-bottom) show possible indications of interparticle cementation. Bar scale on micrographs A and D equals $1 \mu \mathrm{m}$ and on B and C it equals $2 \mu \mathrm{m}$.

proximately 0.4 . Plastic limits remain relatively constant about an average value of $54 \%$, whereas the liquid limits decrease to a low of $140 \%$ at $23 \mathrm{~m}$ sub-bottom and then begin to increase again through the transitional zone to Subunit IB.

Liquid and plastic limit as well as plasticity index $\left(I_{\mathrm{P}}\right)$ values are consistently higher in Subunit IB than in either Subunit IA above or Unit II below (Fig. 7). This is also evident on the standard plasticity chart (Fig. 9; Casagrande, 1948) which shows that the changes in Atterberg limits correspond with the major lithologic divisions. As in Subunit IA, increases and decreases in liquid and plastic limit values in Subunit IB correspond with zones of increasing and decreasing water content. Liquid and plastic limit values average 205 and $82 \%$, respectively.
Liquidity indices (Fig. 8) are quite uniform within Subunit IB, averaging 0.32 . This region of uniform $I_{L}$ values coincides almost exactly with the depth interval represented by Subunit IB ( 28 to $55 \mathrm{~m}$ sub-bottom), suggesting that Subunit IB can be clearly distinguished using the liquidity index.

Atterberg limit data clearly define the contact between Unit I and the underlying carbonate ooze of Unit II. Liquid and plastic limit values for the oozes, averaging 54 and $30 \%$, respectively, are lower in Unit II than in Unit I. Unit II carbonate sediments occur in the lowplasticity region of the standard plasticity chart (Fig. 9), which reflects the difference in the geotechnical behavior between the lower plasticity carbonate oozes and the higher plasticity pelagic clays. One Atterberg limit test on an interbedded pelagic clay interval at $64 \mathrm{~m}$ indicates 

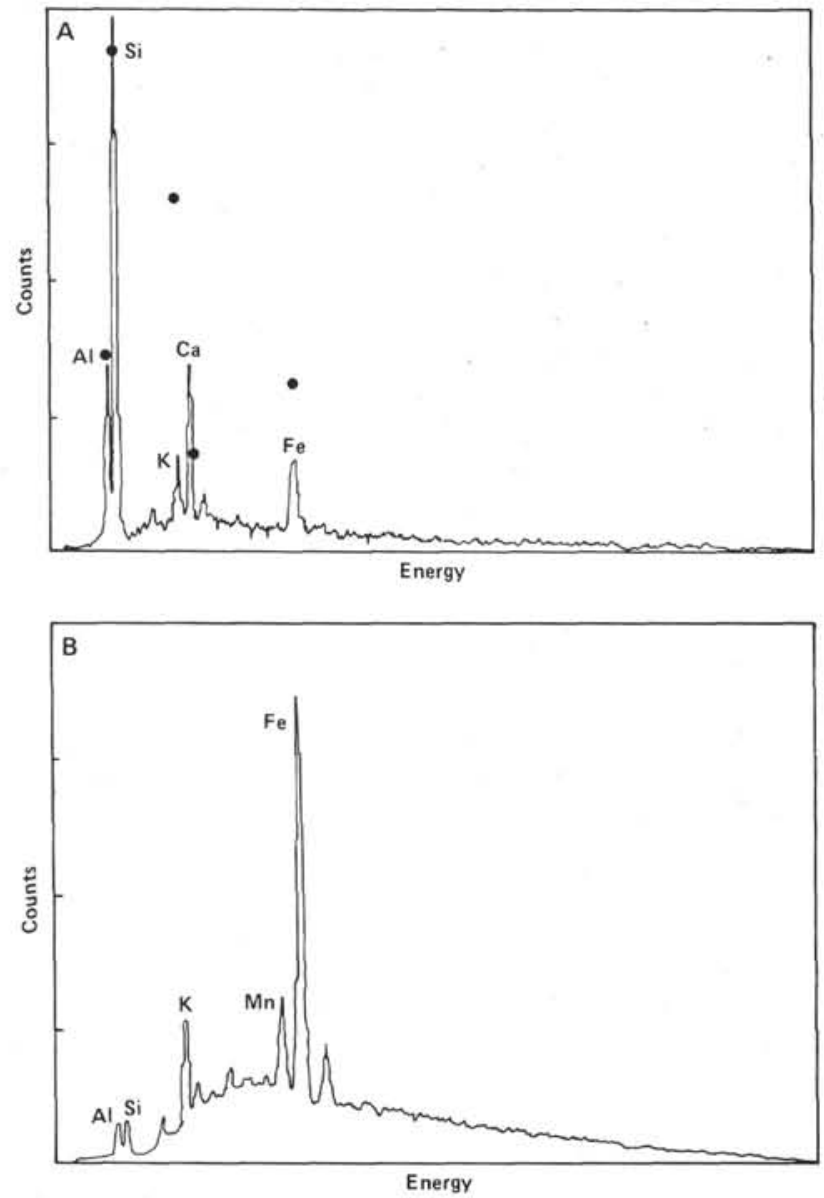

Figure 4. Electron dispersive X-ray spectrometry (EDS) results. (A) EDS results obtained on individual particles present at $7.25 \mathrm{~m}$ (solid line) and at the particle contacts (dots) which suggest interparticle cementation. (B) EDS results from intergrown domains present at $15.56 \mathrm{~m}$ sub-bottom showing iron enrichment which also suggests possible interparticle cementation.

that these interbedded clays are very similar to the pelagic clays tested in Subunit IB. Liquid limits, for the most part, do not exceed the natural water content, resulting in higher liquidity indices for Unit II sediments than for the overlying Subunit IB sediments.

\section{Grain Size and Activity}

Grain-size data vary $\pm 10 \%$ around the mean for Hole 576A sediments (Fig. 10). This variability is attributed to natural variations in the sediment components accumulating in this area and slight differences in interlaboratory test procedures. From the seafloor to approximately $12 \mathrm{~m}$ sub-bottom, the silt content increases from about 34 to $45 \%$; whereas sand percentages vary between 0 and $7 \%$. This general downward coarsening trend to $12 \mathrm{~m}$ sub-bottom appears anomalous because water content and liquid limit increase over this same interval. The normal trend generally shows water content increasing as grain size decreases. This coarsening trend, plus increasing values of activity over this interval (Fig. 11, $\sim 0.9$ to 2.7 ), suggest that the mineralogy may vary significantly from that reported in the preliminary mineralogical analyses. An alternative explanation may be that

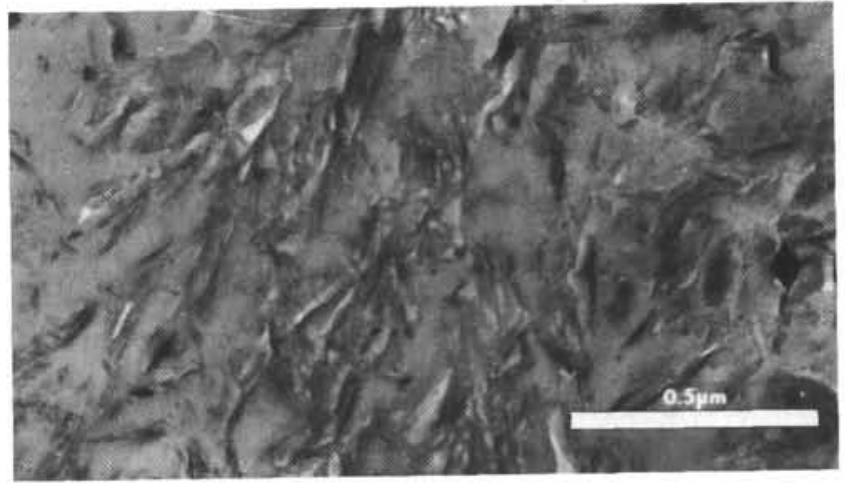

A

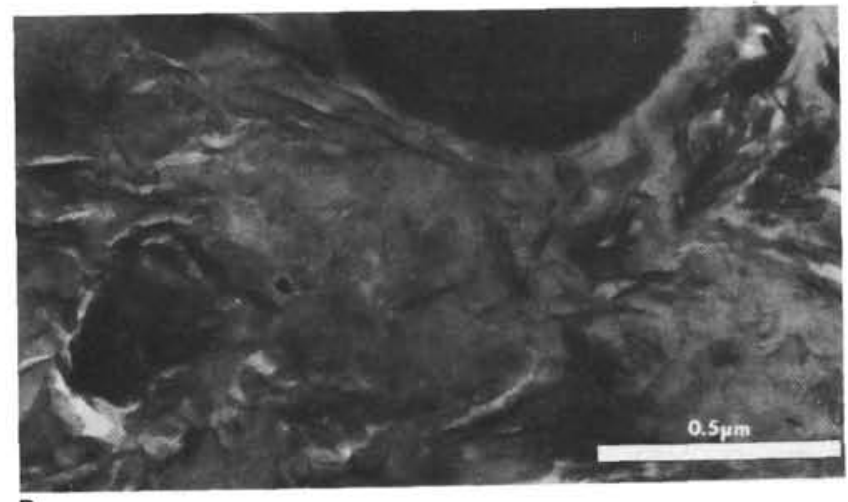

B

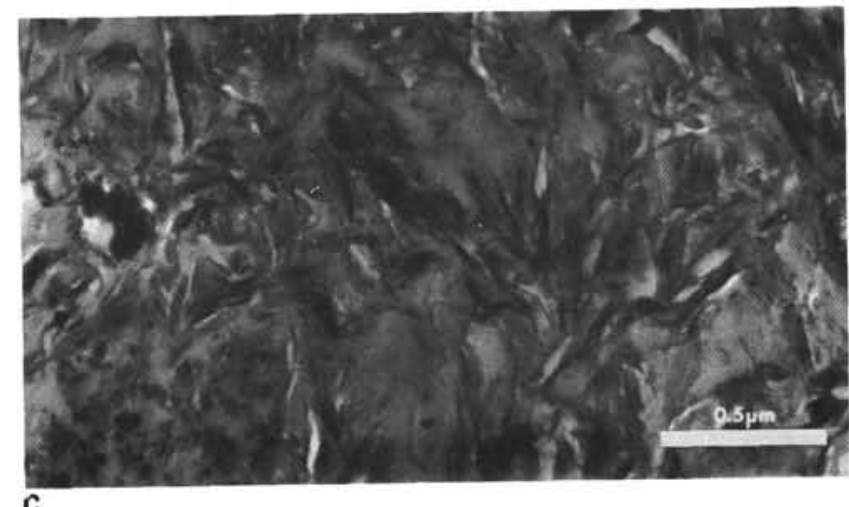

Figure 5. Transmission electron micrographs of sediments located at $22.17 \mathrm{~m} \mathrm{(A)}$ and $46.02 \mathrm{~m}$ (B and $C$ ) sub-bottom showing a complex clay microstructure of particle domains, floccules, and zeolitic material. Large semispherical feature at the top of B is microcrystalline zeolitic material.

the clay-sized fraction was not completely dispersed prior to obtaining aliquots for size analysis. In either case, these unusual trends are consistent with consolidation and shear-strength results presented later in this chapter.

From approximately $12 \mathrm{~m}$ to the base of Subunit IA at $28 \mathrm{~m}$ sub-bottom (Fig. 10), the clay-sized fraction gradually increases as the silt-sized fraction decreases. The gradual increase in the clay-sized fraction probably reflects a decrease in eolian input to this area and marks the transition from Subunit IA to Subunit IB (Site 576 chapter, this volume). Activity becomes less variable through this transition interval with values approaching those evident near the seafloor $(\sim 1.1)$. These values are more typical of the relationship one normally expects between grain size and plasticity. 


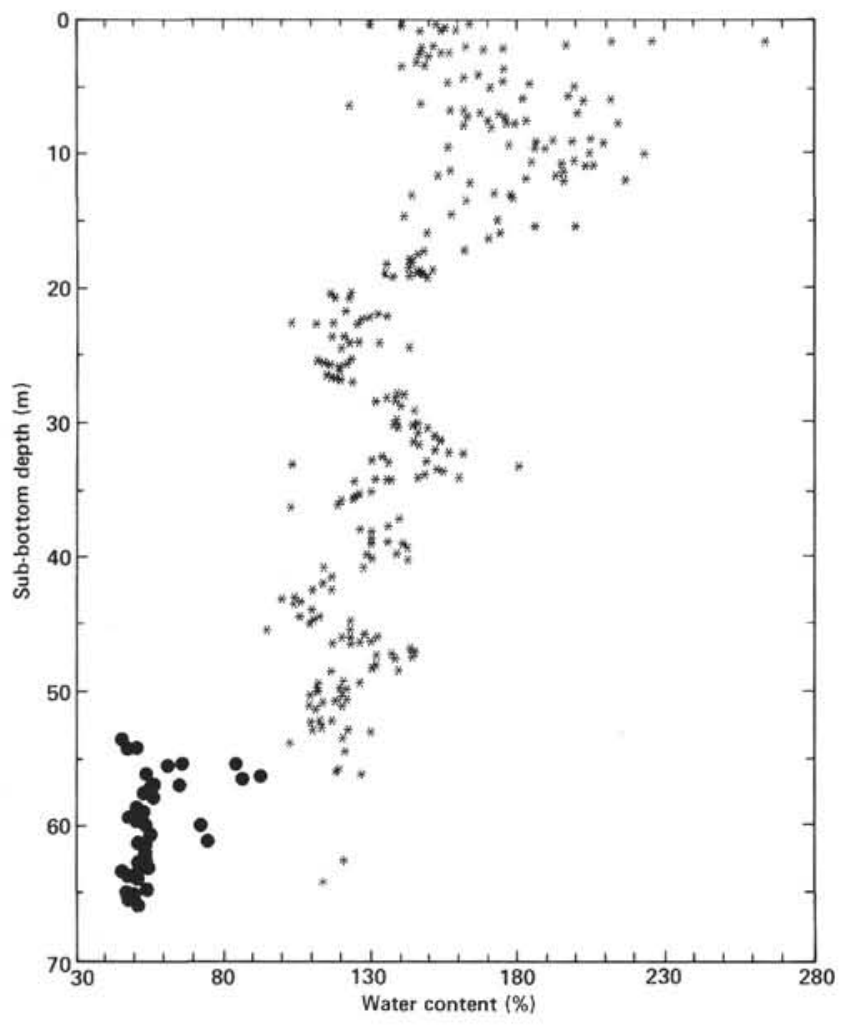

Figure 6. Water content-depth profile for Hole 576A sediments. Solid circles ( $\bullet$ ) below $55 \mathrm{~m}$ represent values obtained in carbonate ooze ( $\%$ dry weight; salt corrected for $35 \%$ ).

From the transitional material of Subunit IA ( 20 to $28 \mathrm{~m}$ sub-bottom) to the pelagic clay in Subunit IB, the amount of clay-sized material reaches a maximum and then remains relatively constant (Fig. $10 ; \sim 85 \%$ claysized fraction). Although some scatter in the grain-size data exist, within certain intervals of Subunit IB the anomalous correlation between grain size, water content, and plasticity identified in Subunit IA is also evident. This is most apparent between 40 and $50 \mathrm{~m}$ sub-bottom. As the likelihood of a large silt- and sand-sized fraction is minimal within these authigenic clays, the most likely explanation for these relationships is that the coarse fraction consists of bonded or cemented clay aggregates and that the dispersion technique used in the size analyses (Folk, 1974) is not adequate for these aggregates. As mentioned earlier, clay-fabric analyses show indications of cementation in these sediments.

A large increase in the silt-sized fraction occurs at the contact between Subunit IB and Unit II. Below this contact, the grain-size profile in the carbonate sediments becomes more uniform with an average of $54 \%$ silt. Interbedded pelagic clay layers contain approximately $75 \%$ clay, with the remainder being silt. Activity values clearly define the carbonates and clays within Unit II (Fig. 11). In general, the carbonate activity values are the lowest observed in Hole 576A sediments.

\section{Bulk Density}

Increasing water content normally corresponds with decreasing bulk density in sediments having constant specific gravity. However, no clear decreasing trend is evi- dent in the upper $12 \mathrm{~m}$ of Subunit IA (Fig. 12 and Table 3). This may result from variable mineralogy or specific gravity related to the increasing grain size through the interval. From $12 \mathrm{~m}$ sub-bottom depth to the base of Subunit IA, increases in bulk density correspond to decreases in water content. This general trend continues down through Subunit IB where bulk density increases to a maximum of $1.50 \mathrm{Mg} / \mathrm{m}^{3}$.

A large break in the bulk-density profile marks the top of Unit II at $55 \mathrm{~m}$ sub-bottom (Fig. 12). Values in this unit are variable but are the highest observed in Hole $576 \mathrm{~A}$, averaging $1.71 \mathrm{Mg} / \mathrm{m}^{3}$. No trend with depth is readily apparent in Unit II, possibly because of the difficulty in obtaining an undisturbed sample in calcareous sediments. The higher bulk-density values occur in the carbonate oozes.

The measured bulk-density values were used to calculate the effective overburden stress-depth profile for Hole 576A sediments, assuming hydrostatic conditions (Fig. 13; Shephard et al., 1978). This profile was used with consolidation test results to determine the state of consolidation.

\section{Compressional Velocity}

Compressional velocity data display a large degree of scatter throughout the recovered sedimentary section (Fig. 14 and Table 3). Values average $1489 \mathrm{~m} / \mathrm{s}(\sigma= \pm 5.5 \mathrm{~m} / \mathrm{s})$ in Subunit IA and $1496 \mathrm{~m} / \mathrm{s}(\sigma= \pm 4.0 \mathrm{~m} / \mathrm{s})$ in Subunit IB. These velocities approximate the velocity of seawater. Velocity data in Unit II show a pronounced increase to $1536 \mathrm{~m} / \mathrm{s}(\sigma= \pm 15.50 \mathrm{~m} / \mathrm{s})$, corresponding to the change in lithology from the overlying pelagic clays to the underlying carbonate oozes.

\section{Summary}

Sediment characterization and index-property data delineate three well-defined units that appear to be consistent with the major lithologic divisions delineated in the Site 576 chapter (this volume). Sediments from Subunit IA (0-28 m sub-bottom) have water contents that increase from the seafloor to approximately $12 \mathrm{~m}$ sub-bottom and then gradually decrease to $28 \mathrm{~m}$ sub-bottom. Over this same interval $(0-28 \mathrm{~m})$ grain size and activity display similar trends, resulting in anomalous indexproperty relationships. Although inconsistent with accepted geotechnical relationships, these results are supported by the consolidation and shear strength data presented later. Liquidity indices are generally greater than 1.0 in the upper $20 \mathrm{~m}$, indicating possible overconsolidation to at least that depth. Limited mineralogy results indicate that illite is the dominant clay mineral present.

Results from Subunit IB (28-55 m sub-bottom) display a marked change from the overlying sediments in Subunit IA. Water contents gradually decrease with depth and grain size appears much more uniform. Changes in bulk density are well correlated with the changes in water content. Atterberg limit results show much higher plasticity indices and lower liquidity indices than in the overlying sediments in Subunit IA. Mineralogy results show the amount of X-ray amorphous material in Subunit IB increases markedly over that present in Subunit IA. 
Table 3. Summary of sediment characterization and index-property analyses performed on Hole 576A sediments.

\begin{tabular}{|c|c|c|c|c|c|c|c|c|}
\hline Core-Section & $\begin{array}{l}\text { Sub-bottom } \\
\text { depth } \\
\text { (m) }\end{array}$ & $\begin{array}{c}\text { Water } \\
\text { content } \\
(\%)\end{array}$ & $\begin{array}{c}\text { Bulk } \\
\text { density } \\
\left(\mathrm{Mg} / \mathrm{m}^{3}\right)\end{array}$ & $\begin{array}{c}\text { Compress. } \\
\text { velocity } \\
(\mathrm{m} / \mathrm{s})\end{array}$ & $\begin{array}{c}\text { Shear } \\
\text { strength } \\
(\mathrm{kPa})\end{array}$ & $\begin{array}{c}\text { Liquid } \\
\text { limit } \\
(\%)\end{array}$ & $\begin{array}{l}\text { Plastic } \\
\text { limit } \\
(\%)\end{array}$ & $\begin{array}{c}\text { Liquidity } \\
\text { index }\end{array}$ \\
\hline $1-1$ & 0.14 & & & & 1.96 & & & \\
\hline $1-1$ & 0.17 & & & & 1.18 & & & \\
\hline $1-1$ & 0.23 & & & & 1.86 & & & \\
\hline $1-1$ & 0.24 & 163 & 1.33 & & & & & \\
\hline $1-1$ & 0.26 & 164 & & 1482 & & & & \\
\hline $1-1$ & 0.33 & 152 & & & 6.37 & & & \\
\hline $1-1$ & 0.42 & & & 1496 & & & & \\
\hline $1-1$ & 0.45 & 130 & & & & & & \\
\hline $1-1$ & 0.47 & & & & 4.5 & & & \\
\hline $1-1$ & 0.49 & 130 & 1.38 & & & & & \\
\hline $1-1$ & 0.55 & 140 & & & 8.13 & & & \\
\hline $1-1$ & 0.58 & & & 1483 & & & & \\
\hline $1-1$ & 0.65 & 155 & & & 5.68 & & & \\
\hline $1-1$ & 0.74 & 159 & 1.33 & & 5.29 & & & \\
\hline $1-1$ & 0.89 & 153 & 1.34 & & 5.88 & & & \\
\hline $1-1$ & 0.93 & 147 & & & & & & \\
\hline $1-1$ & 1.34 & & & & 5.29 & & & \\
\hline $1-2$ & 1.64 & 225 & 1.25 & & & & & \\
\hline $1-2$ & 1.65 & & & & 3.63 & & & \\
\hline $1-2$ & 1.67 & & & 1487 & & & & \\
\hline $1-2$ & 1.70 & 211 & & & & & & \\
\hline $1-2$ & 1.75 & & & & 4.21 & & & \\
\hline $1-2$ & 1.80 & 211 & & & & & & \\
\hline $1-2$ & 1.84 & & & 1502 & 2.65 & & & \\
\hline $1-2$ & 1.89 & 263 & 1.24 & & & & & \\
\hline $1-2$ & 1.94 & & & 1491 & & & & \\
\hline $1-2$ & 1.98 & 196 & & & 6.96 & & & \\
\hline $1-2$ & 2.08 & 162 & & & 10 & & & \\
\hline $1-2$ & 2.09 & & & 1484 & & & & \\
\hline $1-2$ & 2.14 & 151 & 1.36 & & 7.74 & & & \\
\hline $1-2$ & 2.16 & 175 & 1.34 & 1482 & & & & \\
\hline $1-2$ & 2.20 & & & 1485 & & & & \\
\hline $1-2$ & 2.25 & 147 & & & 14.41 & & & \\
\hline $1-2$ & 2.35 & 168 & & & 8.43 & & & \\
\hline $1-2$ & 2.39 & & & 1486 & & & & \\
\hline $1-2$ & 2.44 & 156 & 1.37 & & 5.29 & & & \\
\hline $1-2$ & 2.45 & 153 & 1.34 & & & 131 & 74 & 1.39 \\
\hline $1-2$ & 2.59 & 146 & 1.37 & & 9.6 & & & \\
\hline $1-2$ & 2.84 & 146 & 1.36 & & & & & \\
\hline $1-3$ & 3.00 & 149 & & & & 127 & 47 & 1.3 \\
\hline $1-3$ & 3.24 & & & & 10.39 & & & \\
\hline $1-3$ & 3.34 & 146 & 1.36 & & 7.74 & & & \\
\hline $1-3$ & 3.58 & & & & & & & \\
\hline $1-3$ & 3.60 & & & & & 150 & 49 & 0.93 \\
\hline $1-3$ & 3.84 & 140 & 1.37 & & 6.37 & & & \\
\hline $1-3$ & 3.86 & 149 & & & & & & \\
\hline $1-3$ & 3.93 & & & & & 135 & 67 & 1.37 \\
\hline $1-3$ & 4.02 & 175 & 1.29 & 1484 & & & & \\
\hline $1-3$ & 4.14 & & & & 10.68 & & & \\
\hline $1-4$ & 4.52 & 166 & 1.34 & & & & & \\
\hline $1-4$ & 4.66 & & & 1476 & & & & \\
\hline $1-4$ & 4.71 & & & 1478 & & & & \\
\hline $1-4$ & 4.73 & 161 & & & 9.31 & & & \\
\hline $1-4$ & 4.85 & 175 & & & 13.62 & & & \\
\hline $1-4$ & 4.88 & & & 1498 & & & & \\
\hline $1-4$ & 4.94 & 156 & 1.34 & & 12.25 & & & \\
\hline$i-4$ & 4.98 & & & & & 121 & 44 & 1.46 \\
\hline $1-4$ & 5.24 & 170 & 1.32 & & 8.23 & & & \\
\hline $1-4$ & 5.25 & 183 & & & & & & \\
\hline 1.4 & 5.50 & 199 & & & & 148 & 75 & 1.7 \\
\hline $1-4$ & 6.01 & & & 1485 & & & & \\
\hline $1-4$ & 6.05 & 197 & & & 11.17 & & & \\
\hline $1-4$ & 6.15 & 181 & & & 9.02 & & & \\
\hline $1-4$ & 6.18 & & & 1483 & & & & \\
\hline $1-4$ & 6.24 & 202 & 1.29 & & 7.15 & & & \\
\hline $1-4$ & 6.30 & 211 & & & & & 51 & 1.9 \\
\hline $1-4$ & 6.49 & 147 & 1.36 & & 14.41 & & & \\
\hline 1.4 & 6.52 & & & 1489 & & & & \\
\hline $1-4$ & 6.74 & 122 & 1.41 & & 6.37 & & & \\
\hline $1-4$ & 6.99 & 162 & 1.34 & & 9.6 & & & \\
\hline 1.5 & 7.03 & & & 1496 & & & & \\
\hline $1-5$ & 7.05 & 157 & & & 15.68 & & & \\
\hline 1.5 & 7.15 & 200 & & & 8.53 & & & \\
\hline $1-5$ & 7.21 & & & 1488 & & & & \\
\hline $1-5$ & 7.24 & 173 & 1.33 & & 9.31 & & & \\
\hline
\end{tabular}


Table 3. (Continued).

\begin{tabular}{|c|c|c|c|c|c|c|c|c|}
\hline Core-Section & $\begin{array}{l}\text { Sub-bottom } \\
\text { depth } \\
\text { (m) }\end{array}$ & $\begin{array}{c}\text { Water } \\
\text { content } \\
(\%)\end{array}$ & $\begin{array}{c}\text { Bulk } \\
\text { density } \\
\left(\mathrm{Mg} / \mathrm{m}^{3}\right)\end{array}$ & $\begin{array}{l}\text { Compress. } \\
\text { velocity } \\
(\mathrm{m} / \mathrm{s})\end{array}$ & $\begin{array}{c}\text { Shear } \\
\text { strength } \\
(\mathrm{kPa})\end{array}$ & $\begin{array}{l}\text { Liquid } \\
\text { limit } \\
(\%)\end{array}$ & $\begin{array}{l}\text { Plastic } \\
\text { limit } \\
(\%)\end{array}$ & $\begin{array}{l}\text { Liquidity } \\
\text { index }\end{array}$ \\
\hline $1-5$ & 7.27 & 167 & & & & 135 & 39 & 1.33 \\
\hline 1.5 & 7.28 & & & & & & & \\
\hline $1-5$ & 7.36 & 163 & & & & & & \\
\hline $1-5$ & 7.54 & 175 & 1.35 & & & & & \\
\hline $1-5$ & 7.67 & & & & & 118 & 42 & \\
\hline $1-5$ & 7.70 & 170 & & & & & & \\
\hline $1-5$ & 7.82 & 183 & 1.37 & 1482 & & & & \\
\hline $1-5$ & 7.85 & 176 & & & 5.88 & & & \\
\hline $1-5$ & 7.95 & 176 & & & 16.27 & & & \\
\hline $1-5$ & 8.01 & & & 1486 & & & & \\
\hline $1-5$ & 8.04 & 214 & 1.27 & & 5.88 & & & \\
\hline $1-5$ & 8.07 & & & 1486 & & & & \\
\hline $1-5$ & 8.10 & 179 & & & 13.82 & & & \\
\hline $1-5$ & 8.20 & 171 & & & 18.62 & & & \\
\hline $1-5$ & 8.25 & & & 1483 & & & & \\
\hline 1.5 & 8.29 & 161 & 1.35 & & 8.23 & & & \\
\hline $1-5$ & 8.49 & & & & 21.85 & & & \\
\hline $2-1$ & 9.25 & 205 & & 1490 & 4.31 & & & \\
\hline $2-1$ & 9.35 & 192 & & & 7.45 & & & \\
\hline $2-1$ & 9.40 & & & 1496 & & & & \\
\hline $2-1$ & 9.44 & 198 & 1.3 & & 8.82 & & & \\
\hline $2-1$ & 9.47 & 209 & 1.35 & 1488 & & & & \\
\hline $2-1$ & 9.49 & & & 1494 & & & & \\
\hline $2-1$ & 9.55 & 186 & & & 16.56 & & & \\
\hline $2-1$ & 9.65 & 177 & & & 9.9 & & & \\
\hline $2-1$ & 9.67 & & & 1497 & & & & \\
\hline $2-1$ & 9.69 & 156 & 1.36 & & 9.1 & & & \\
\hline $2-1$ & 9.94 & 189 & 1.3 & & 9.02 & & & \\
\hline $2-1$ & 10.00 & 186 & & & 14.41 & & & \\
\hline $2-1$ & 10.09 & 204 & 1.28 & & 11.17 & & & \\
\hline $2-2$ & 10.22 & & & & & & & \\
\hline $2-2$ & 10.26 & 223 & & & & 154 & 43 & 1.62 \\
\hline $2-2$ & 10.78 & 198 & & & 12.84 & & & \\
\hline $2-2$ & 10.89 & 184 & 1.31 & & 9.6 & & & \\
\hline $2-2$ & 10.92 & 195 & 1.37 & 1487 & & & & \\
\hline $2-2$ & 11.09 & 203 & 1.28 & & 8.53 & & & \\
\hline $2-2$ & 11.11 & 205 & 1.29 & & & & & \\
\hline $2-2$ & 11.44 & 157 & 1.34 & & 10.09 & & & \\
\hline $2-2$ & 11.65 & 195 & & & & & & \\
\hline $2-3$ & 11.86 & 153 & & & & & & \\
\hline $2-3$ & 11.96 & 193 & & & & & & \\
\hline $2-3$ & 11.97 & 153 & 1.4 & 1478 & & & & \\
\hline $2-3$ & 12.06 & & & & 12.25 & & & \\
\hline $2-3$ & 12.16 & & & & 8.82 & & & \\
\hline $2-3$ & 12.19 & 183 & 1.29 & & 5.88 & & & \\
\hline $2-3$ & 12.24 & 216 & & & & & & \\
\hline $2-3$ & 12.42 & 195 & & & & 176 & 36 & 1.13 \\
\hline $2-3$ & 12.63 & 163 & & & & & & \\
\hline $2-3$ & 12.80 & & & & & 167 & 56 & 0.98 \\
\hline $2-3$ & 12.83 & & & & & & & \\
\hline $2-4$ & 13.30 & 171 & & & 49.29 & & & \\
\hline $2-4$ & 13.35 & 144 & & & & & & \\
\hline $2-4$ & 13.40 & 178 & & & 29.3 & & & \\
\hline $2-4$ & 13.49 & 178 & 1.3 & & 10.09 & & & \\
\hline $2-4$ & 13.52 & 179 & 1.33 & & & & & \\
\hline $2-4$ & 13.68 & 162 & & & & & & \\
\hline $2-4$ & 13.73 & & & & & & & \\
\hline $2-4$ & 14.00 & & & & & 170 & 87 & 0.83 \\
\hline $2-5$ & 14.80 & 141 & & & 38.02 & & & \\
\hline $2-5$ & 14.90 & 157 & & & 28.22 & & & \\
\hline $2-5$ & 14.94 & & & 1488 & & & & \\
\hline $2-5$ & 14.99 & 173 & 1.31 & & 11.76 & & & \\
\hline $2-5$ & 15.53 & & & & & & & \\
\hline $2-5$ & 15.54 & 185 & & & & 186 & 43 & 0.99 \\
\hline $2-5$ & 15.61 & 199 & & & & & & \\
\hline $2-6$ & 16.22 & 174 & 1.34 & & & & & \\
\hline $2-6$ & 16.23 & 149 & & & & & & \\
\hline $2-6$ & 16.42 & 170 & & & & 162 & 44 & 1.07 \\
\hline $2-6$ & 16.44 & & & & & & & \\
\hline $2-6$ & 16.80 & & & & & 173 & 62 & 1.1 \\
\hline $2-6$ & 17.39 & 148 & 1.36 & & 19.5 & & & \\
\hline $2-6$ & 17.42 & 161 & 1.41 & 1493 & & & & \\
\hline $2-6$ & 17.69 & 146 & 1.35 & & 13.62 & & & \\
\hline $2-6$ & 17.73 & & & & 11.96 & & & \\
\hline $3-1$ & 18.22 & & & 1488 & & & & \\
\hline $3-1$ & 18.25 & 143 & & & 15.97 & & & \\
\hline
\end{tabular}


Table 3. (Continued).

\begin{tabular}{|c|c|c|c|c|c|c|c|c|}
\hline Core-Section & $\begin{array}{l}\text { Sub-bottom } \\
\text { depth } \\
\text { (m) }\end{array}$ & $\begin{array}{c}\text { Water } \\
\text { content } \\
(\%)\end{array}$ & $\begin{array}{c}\text { Bulk } \\
\text { density } \\
\left(\mathrm{Mg} / \mathrm{m}^{3}\right)\end{array}$ & $\begin{array}{l}\text { Compress. } \\
\text { velocity } \\
(\mathrm{m} / \mathrm{s})\end{array}$ & $\begin{array}{l}\text { Shear } \\
\text { strength } \\
(\mathrm{kPa})\end{array}$ & $\begin{array}{l}\text { Liquid } \\
\text { limit } \\
(\%)\end{array}$ & $\begin{array}{c}\text { Plastic } \\
\text { limit } \\
(\%)\end{array}$ & $\begin{array}{l}\text { Liquidity } \\
\text { index }\end{array}$ \\
\hline $3-1$ & 18.35 & 144 & & & 18.62 & & & \\
\hline $3-1$ & 18.36 & & & 1505 & & & & \\
\hline $3-1$ & 18.44 & 135 & 1.38 & & & & & \\
\hline 3-1 & 18.69 & 143 & 1.36 & & 11.96 & & & \\
\hline $3-1$ & 18.94 & 151 & 1.35 & & 11.96 & & & \\
\hline $3-1$ & 18.97 & 147 & 1.42 & 1479 & & & & \\
\hline $3-1$ & 19.00 & & & 1490 & & & & \\
\hline 3-1 & 19.03 & 146 & & & 20.29 & & & \\
\hline 3-1 & 19.13 & 147 & & & 23.42 & & & \\
\hline $3-1$ & 19.14 & & & 1489 & & & & \\
\hline $3-1$ & 19.19 & 135 & 1.37 & & 16.27 & & & \\
\hline $3-1$ & 19.30 & 143 & & & 19.99 & & & \\
\hline 3-1 & 19.34 & & & 1490 & & & & \\
\hline 3-1 & 19.40 & 148 & & & 20.68 & & & \\
\hline $3-1$ & 19.44 & 137 & 1.36 & & 11.76 & & & \\
\hline $3-1$ & 19.52 & & & & & & & \\
\hline 3-1 & 19.53 & 150 & & & & 145 & 47 & 1.05 \\
\hline $3-2$ & 20.61 & 116 & 1.41 & & & & & \\
\hline $3-2$ & 20.64 & & & 1484 & & & & \\
\hline $3-2$ & 20.67 & 123 & & & 18.03 & & & \\
\hline $3-2$ & 20.69 & & & 1492 & & & & \\
\hline $3-2$ & 20.73 & 118 & 1.48 & 1478 & & & & \\
\hline $3-2$ & 20.74 & & & & 13.03 & & & \\
\hline $3-2$ & 20.76 & 119 & 1.42 & & & 162 & 93 & 0.37 \\
\hline $3-2$ & 20.93 & 123 & & & & & & \\
\hline $3-2$ & 20.97 & & & & & 163 & 80 & 0.51 \\
\hline $3-3$ & 21.80 & 121 & & & & 153 & 98 & 0.4 \\
\hline $3-3$ & 22.14 & 132 & 1.37 & & 14.9 & & & \\
\hline $3-3$ & 22.24 & 135 & & & 21.07 & & & \\
\hline $3-3$ & 22.34 & 135 & & & 21.36 & & & \\
\hline 3-3 & 22.35 & & & 1491 & & & & \\
\hline $3-3$ & 22.39 & 130 & & & 14.11 & & & \\
\hline $3-3$ & 22.42 & & & 1481 & & & & \\
\hline $3-3$ & 22.59 & 127 & 1.39 & & 15.68 & & & \\
\hline $3-4$ & 22.72 & & & 1490 & & & & \\
\hline $3-4$ & 22.74 & 126 & & & 24.89 & & & \\
\hline $3-4$ & 22.80 & & & 1486 & & & & \\
\hline $3-4$ & 22.82 & 102 & & & 23.62 & & & \\
\hline $3-4$ & 22.84 & 103 & 1.44 & & 15.48 & & & \\
\hline $3-4$ & 22.88 & & & & & & & \\
\hline $3-4$ & 22.89 & 111 & & & & 140 & 42 & 0.7 \\
\hline $3-4$ & 22.96 & 117 & & & & & & \\
\hline $3-4$ & 23.77 & 121 & 1.46 & 1485 & & & & \\
\hline $3-4$ & 23.89 & 116 & 1.4 & & 14.9 & & & \\
\hline $3-5$ & 24.25 & 125 & & & 19.99 & & & \\
\hline $3-5$ & 24.27 & & & 1483 & & & & \\
\hline $3-5$ & 24.31 & 122 & 1.4 & & 15.19 & & & \\
\hline $3-5$ & 24.34 & 132 & 1.42 & & & & & \\
\hline $3-5$ & 24.64 & 142 & & & & & & \\
\hline $3-5$ & 24.80 & 119 & & & & 149 & 50 & 0.7 \\
\hline $3-5$ & 25.42 & & & 1487 & & & & \\
\hline $3-5$ & 25.44 & 123 & & & 22.83 & & & \\
\hline $3-5$ & 25.49 & 122 & 1.39 & & 16.27 & & & \\
\hline $3-6$ & 25.74 & 111 & & & 26.66 & & & \\
\hline $3-6$ & 25.75 & & & 1485 & & & & \\
\hline $3-6$ & 25.92 & 115 & & & 28.03 & & & \\
\hline $3-6$ & 25.96 & & & 1490 & & & & \\
\hline $3-6$ & 25.99 & 113 & 1.41 & & 15.48 & & & \\
\hline $3-6$ & 26.02 & 121 & 1.43 & & & 168 & 75 & 0.49 \\
\hline $3-6$ & 26.23 & & & & & 167 & 64 & 0.54 \\
\hline $3-6$ & 26.33 & 119 & & & & & & \\
\hline $3-6$ & 26.57 & 119 & 1.5 & 1486 & & & & \\
\hline $3-6$ & 26.79 & 114 & 1.42 & & 16.56 & & & \\
\hline $3-6$ & 26.85 & & & 1488 & & & & \\
\hline 3-6 & 26.89 & 117 & & & 25.28 & & & \\
\hline $3-6$ & 26.99 & 116 & & & 24.7 & & & \\
\hline 3-6 & 27.00 & & & 1488 & & & & \\
\hline 3-6 & 27.04 & 119 & 1.4 & & 16.56 & & & \\
\hline $3-6$ & 27.19 & 123 & 1.41 & & 17.35 & & & \\
\hline $4-1$ & 27.94 & 138 & 1.34 & & 3.23 & & & \\
\hline $4-1$ & 28.00 & & & 1488 & & & & \\
\hline $4-1$ & 28.15 & 141 & & 1492 & 13.03 & & & \\
\hline $4-1$ & 28.19 & 134 & 1.36 & & 9.6 & & & \\
\hline $4-1$ & 28.35 & 135 & & & 17.64 & & & \\
\hline $4-1$ & 28.39 & & & 1494 & & & & \\
\hline $4-1$ & 28.44 & 138 & 1.36 & & 10.39 & & & \\
\hline
\end{tabular}


Table 3. (Continued).

\begin{tabular}{|c|c|c|c|c|c|c|c|c|}
\hline Core-Section & $\begin{array}{l}\text { Sub-bottom } \\
\text { depth } \\
\text { (m) }\end{array}$ & $\begin{array}{l}\text { Water } \\
\text { content } \\
(\%)\end{array}$ & $\begin{array}{c}\text { Bulk } \\
\text { density } \\
\left(\mathrm{Mg} / \mathrm{m}^{3}\right)\end{array}$ & $\begin{array}{l}\text { Compress. } \\
\text { velocity } \\
(\mathrm{m} / \mathrm{s})\end{array}$ & $\begin{array}{c}\text { Shear } \\
\text { strength } \\
(\mathrm{kPa})\end{array}$ & $\begin{array}{c}\text { Liquid } \\
\text { limit } \\
(\%)\end{array}$ & $\begin{array}{c}\text { Plastic } \\
\text { limit } \\
(\%)\end{array}$ & $\begin{array}{l}\text { Liquidity } \\
\text { index }\end{array}$ \\
\hline $4-1$ & 28.47 & & & 1490 & & & & \\
\hline $4-1$ & 28.50 & & & & & 182 & 71 & 0.7 \\
\hline 4-1 & 28.69 & 131 & 1.37 & & 10.68 & & & \\
\hline $4-1$ & 28.94 & & & & 11.96 & & & \\
\hline 4-1 & 29.09 & 140 & 1.35 & & 9.02 & & & \\
\hline $4-2$ & 29.37 & & & & & & & \\
\hline 4-2 & 29.39 & & & & & & & \\
\hline $4-2$ & 29.46 & 144 & & & & 185 & 48 & 0.7 \\
\hline $4-2$ & 29.47 & & & & & & & \\
\hline $4-2$ & 29.53 & & & & & & & \\
\hline $4-2$ & 29.75 & 139 & & & & & & \\
\hline $4-2$ & 30.09 & 138 & 1.4 & & & 186 & 77 & 0.56 \\
\hline $4-2$ & 30.25 & 146 & 1.36 & & & & & \\
\hline $4-2$ & 30.34 & 143 & & & 18.62 & 220 & 112 & 0.29 \\
\hline $4-2$ & 30.38 & & & 1492 & & & & \\
\hline $4-2$ & 30.40 & 137 & & & & 213 & 101 & 0.3 \\
\hline $4-2$ & 30.42 & 139 & & & 10.39 & & & \\
\hline $4-2$ & 30.65 & 149 & & & & & & \\
\hline $4-3$ & 30.73 & 145 & & & & & & \\
\hline $4-3$ & 31.22 & 150 & 1.33 & & 11.96 & & & \\
\hline $4-3$ & 31.46 & 153 & 1.31 & & & & & \\
\hline $4-3$ & 31.51 & & & & 18.62 & & & \\
\hline $4-3$ & 31.52 & 154 & & & & & & \\
\hline $4-3$ & 31.54 & & & 1494 & & & & \\
\hline $4-3$ & 31.63 & 153 & & & 24.79 & & & \\
\hline $4-3$ & 31.67 & 144 & 1.35 & & 15.68 & & & \\
\hline $4-3$ & 31.82 & 146 & 1.35 & & 15.48 & & & \\
\hline $4-4$ & 32.24 & & & 1499 & & & & \\
\hline $4-4$ & 32.31 & 151 & & & 34.01 & & & \\
\hline $4-4$ & 32.39 & & & 1498 & & & & \\
\hline $4-4$ & 32.46 & 156 & 1.34 & & 18.62 & & & \\
\hline $4-4$ & 32.47 & 161 & 1.35 & & & & & \\
\hline $4-4$ & 32.75 & & & 1497 & & & & \\
\hline $4-4$ & 32.87 & 134 & & & 33.32 & & & \\
\hline $4-4$ & 32.96 & 130 & 1.38 & & 20.48 & & & \\
\hline $4-4$ & 33.01 & & & 1495 & & & & \\
\hline $4-4$ & 33.07 & 148 & & & 32.83 & & & \\
\hline $4-4$ & 33.19 & 136 & & & 35.28 & & & \\
\hline $4-4$ & 33.21 & & & 1495 & & & & \\
\hline $4-4$ & 33.26 & 102 & 1.33 & & 22,34 & & & \\
\hline $4-4$ & 33.31 & & & 1494 & & & & \\
\hline $4-4$ & 33.55 & 180 & & & 35.97 & & & \\
\hline 4-5 & 33.77 & 152 & & & 39.69 & & & \\
\hline $4-5$ & 33.81 & & & 1502 & & & & \\
\hline $4-5$ & 33.92 & & & & 22.34 & & & \\
\hline $4-5$ & 33.95 & 155 & 1.4 & 1492 & & & & \\
\hline $4-5$ & 34.03 & & & 1494 & & & & \\
\hline 4-5 & 34.07 & 148 & & & 36.26 & & & \\
\hline $4-5$ & 34.17 & 146 & 1.34 & & 21.85 & & & \\
\hline 4-5 & 34.24 & & & 1497 & & & & \\
\hline $4-5$ & 34.29 & 159 & & & 33.32 & & & \\
\hline $4-5$ & 34.39 & & 1.36 & & 54 & & & \\
\hline $4-5$ & 34.43 & & & & & & & \\
\hline 4-5 & 34.47 & 135 & & & & 236 & 82 & 0.34 \\
\hline $4-5$ & 34.50 & 136 & & & & 209 & 73 & 0.5 \\
\hline $4-5$ & 34.54 & 131 & & & & & & \\
\hline $4-5$ & 34.56 & 124 & & & & & & \\
\hline $4-5$ & 34.62 & & & & & 217 & 105 & 0.23 \\
\hline $4-6$ & 35.29 & 130 & 1.42 & & & 211 & 81 & 0.38 \\
\hline $4-6$ & 35.41 & 127 & & & 38.61 & & & \\
\hline $4-6$ & 35.47 & & & 1500 & & & & \\
\hline $4-6$ & 35.54 & 125 & 1.39 & & 60 & & & \\
\hline $4-6$ & 35.82 & 124 & 1.43 & 1493 & & & & \\
\hline $4-6$ & 35.97 & 120 & 1.4 & & 21.85 & & & \\
\hline $4-6$ & 36.21 & & & & 37 & & & \\
\hline $4-6$ & 36.23 & 119 & 1.41 & & & & & \\
\hline $4-6$ & 36.29 & & & 1496 & & & & \\
\hline $4-6$ & 36.34 & & & & 24.3 & & & \\
\hline $4-6$ & 36.36 & 102 & & & & & & \\
\hline $4-6$ & 36.42 & & & 1495 & & & & \\
\hline $5-1$ & 37.29 & 139 & & & & & & \\
\hline $5-1$ & 37.30 & & & & 17.35 & & & \\
\hline $5-1$ & 37.32 & & & 1492 & & & & \\
\hline $5-1$ & 37.44 & 139 & 1.35 & & 28 & & & \\
\hline $5-1$ & 37.86 & & & & 33 & & & \\
\hline $5-1$ & 37.95 & & & & 24.89 & & & \\
\hline
\end{tabular}


Table 3. (Continued).

\begin{tabular}{|c|c|c|c|c|c|c|c|c|}
\hline Core-Section & $\begin{array}{l}\text { Sub-bottom } \\
\text { depth } \\
\text { (m) }\end{array}$ & $\begin{array}{c}\text { Water } \\
\text { content } \\
(\%)\end{array}$ & $\begin{array}{c}\text { Bulk } \\
\text { density } \\
\left(\mathrm{Mg} / \mathrm{m}^{3}\right)\end{array}$ & $\begin{array}{l}\text { Compress. } \\
\text { velocity } \\
(\mathrm{m} / \mathrm{s})\end{array}$ & $\begin{array}{l}\text { Shear } \\
\text { strength } \\
(\mathrm{kPa})\end{array}$ & $\begin{array}{l}\text { Liquid } \\
\text { limit } \\
(\%)\end{array}$ & $\begin{array}{c}\text { Plastic } \\
\text { limit } \\
(\% 0)\end{array}$ & $\begin{array}{l}\text { Liquidity } \\
\text { index }\end{array}$ \\
\hline $5-1$ & 37.97 & 136 & & & & & & \\
\hline $5-1$ & 38.08 & 127 & 1.46 & 1482 & & & & \\
\hline $5-1$ & 38.15 & & & & 22.93 & & & \\
\hline $5-1$ & 38.17 & 130 & & & & & & \\
\hline $5-1$ & 38.28 & & & 1494 & & & & \\
\hline $5-1$ & 38.34 & 130 & 1.37 & & 44 & & & \\
\hline $5-1$ & 38.59 & 130 & 1.37 & & 29 & & & \\
\hline $5-1$ & 38.73 & & & & 30.67 & & & \\
\hline $5-2$ & 38.94 & 130 & 1.37 & & 34 & & & \\
\hline $5-2$ & 39.10 & & & & 25.09 & & & \\
\hline $5-2$ & 39.12 & 136 & 1.37 & & & & & \\
\hline $5-2$ & 39.20 & 141 & & & & & & \\
\hline $5-2$ & 39.23 & & & 1493 & & & & \\
\hline $5-2$ & 39.24 & & & & 36 & & & \\
\hline $5-2$ & 39.37 & 142 & 1.38 & & & 224 & 96 & 0.36 \\
\hline $5-2$ & 39.74 & 129 & 1.38 & & 30 & & & \\
\hline $5-2$ & 40.00 & 139 & & & 23.72 & & & \\
\hline $5-2$ & 40.09 & & & 1493 & & & & \\
\hline $5-2$ & 40.19 & 130 & & & 40.19 & & & \\
\hline $5-3$ & 40.28 & & & & & & & \\
\hline $5-3$ & 40.36 & 142 & 1.37 & & & 229 & 96 & 0.35 \\
\hline $5-3$ & 40.74 & & & & 54 & & & \\
\hline $5-3$ & 41.02 & 127 & 1.42 & & & 210 & 74 & 0.39 \\
\hline $5-3$ & 41.07 & 114 & 1.48 & 1485 & & & & \\
\hline $5-3$ & 41.29 & & & & 49 & & & \\
\hline $5-3$ & 41.59 & & & & 49 & & & \\
\hline $5-4$ & 41.73 & 116 & & & & & & \\
\hline $5-4$ & 41.94 & & & & & 208 & 83 & 0.26 \\
\hline $5-4$ & 42.02 & 115 & 1.45 & & & 205 & 85 & 0.25 \\
\hline $5-4$ & 42.25 & & & & 34.69 & & & \\
\hline $5-4$ & 42.65 & 117 & & & & & & \\
\hline $5-4$ & 42.73 & 110 & & & & 166 & 64 & 0.45 \\
\hline $5-4$ & 42.78 & & & & & & & \\
\hline $5-4$ & 42.89 & & & & & & & \\
\hline $5-4$ & 42.91 & & & & & & & \\
\hline $5-5$ & 43.23 & 104 & & & & & & \\
\hline $5-5$ & 43.25 & & & 1501 & & & & \\
\hline 5.5 & 43.29 & 101 & 1.46 & & 38 & & & \\
\hline $5-5$ & 43.37 & & & & & & & \\
\hline $5-5$ & 43.38 & 106 & & & & 170 & 58 & 0.43 \\
\hline $5-5$ & 43.39 & 105 & 1.5 & 1487 & & & & \\
\hline $5-5$ & 43.52 & & & 1504 & & & & \\
\hline $5-5$ & 43.54 & 104 & & & 37.34 & & & \\
\hline $5-5$ & 43.59 & 104 & 1.46 & & 46 & & & \\
\hline $5-5$ & 43.79 & & & & & 184 & 61 & 0.38 \\
\hline $5-5$ & 44.00 & 111 & & & & 197 & 73 & 0.31 \\
\hline $5-5$ & 44.43 & & & & & & & \\
\hline $5-5$ & 44.57 & & & & 81 & & & \\
\hline $5-5$ & 44.59 & 106 & 1.44 & & & & & \\
\hline $5-5$ & 44.61 & 113 & 1.46 & & & 184 & 74 & 0.35 \\
\hline $5-6$ & 44.80 & 111 & & & 36.65 & & & \\
\hline $5-6$ & 44.86 & & & 1498 & & & & \\
\hline $5-6$ & 44.94 & 109 & 1.44 & & 17 & & & \\
\hline $5-6$ & 44.97 & 123 & 1.43 & & & 202 & 79 & 0.36 \\
\hline $5-6$ & 45.59 & 94 & 1.3 & & 86 & & & \\
\hline $5-6$ & 45.60 & 123 & 1.41 & & & 218 & 88 & 0.27 \\
\hline $5-6$ & 45.77 & 128 & 1.47 & 1490 & & & & \\
\hline $5-6$ & 45.99 & 123 & 1.4 & & 94 & & & \\
\hline $5-6$ & 46.09 & 132 & & & 39.3 & & & \\
\hline $5-6$ & 46.13 & & & 1496 & & & & \\
\hline $5-6$ & 46.19 & 120 & 1.41 & & 81 & & & \\
\hline $5-7$ & 46.35 & 130 & & 1495 & & & & \\
\hline $5-7$ & 46.44 & 123 & 1.4 & & 26.66 & & & \\
\hline $5-7$ & 46.47 & 125 & 1.44 & 1493 & & & & \\
\hline $5-7$ & 46.69 & 118 & 1.41 & & 36 & & & \\
\hline $6-1$ & 46.94 & 142 & 1.29 & & 22 & & & \\
\hline $6-1$ & 46.98 & 144 & & & & & & \\
\hline $6-1$ & 47.20 & 145 & 1.37 & & & 246 & 91 & 0.35 \\
\hline $6-1$ & 47.37 & 137 & 1.37 & 1492 & & & & \\
\hline $6-1$ & 47.42 & 144 & & & 26.66 & & & \\
\hline $6-1$ & 47.52 & & & 1491 & & & & \\
\hline $6-1$ & 47.59 & 132 & 1.37 & & 33 & & & \\
\hline $6-1$ & 47.60 & 138 & & & & & & \\
\hline $6-1$ & 47.67 & & & 1495 & & & & \\
\hline $6-2$ & 47.70 & & & & 31.75 & & & \\
\hline $6-2$ & 47.84 & & & & 65 & & & \\
\hline
\end{tabular}


Table 3. (Continued).

\begin{tabular}{|c|c|c|c|c|c|c|c|c|}
\hline Core-Section & $\begin{array}{l}\text { Sub-bottom } \\
\text { depth } \\
\text { (m) }\end{array}$ & $\begin{array}{l}\text { Water } \\
\text { content } \\
(\%)\end{array}$ & $\begin{array}{c}\text { Bulk } \\
\text { density } \\
\left(\mathrm{Mg} / \mathrm{m}^{3}\right)\end{array}$ & $\begin{array}{l}\text { Compress. } \\
\text { velocity } \\
(\mathrm{m} / \mathrm{s})\end{array}$ & $\begin{array}{c}\text { Shear } \\
\text { strength } \\
(\mathrm{kPa})\end{array}$ & $\begin{array}{l}\text { Liquid } \\
\text { limit } \\
(\%)\end{array}$ & $\begin{array}{l}\text { Plastic } \\
\text { limit } \\
(\%)\end{array}$ & $\begin{array}{l}\text { Liquidity } \\
\text { index }\end{array}$ \\
\hline $6-2$ & 48.21 & 132 & 1.42 & & & 226 & 97 & 0.27 \\
\hline $6-2$ & 48.44 & 130 & 1.38 & & 81 & & & \\
\hline $6-2$ & 48.47 & 131 & 1.43 & & & & & \\
\hline $6-2$ & 48.55 & 139 & & & 30.97 & & & \\
\hline $6-2$ & 48.61 & & & 1497 & & & & \\
\hline $6-2$ & 48.69 & 116 & 1.42 & & 89 & & & \\
\hline $6-2$ & 48.80 & & & & & 227 & 71 & 0.3 \\
\hline $6-2$ & 48.95 & & & & & & & \\
\hline $6-2$ & 49.40 & 121 & & & 35.48 & & & \\
\hline $6-2$ & 49.48 & & & 1497 & & & & \\
\hline $6-2$ & 49.59 & 112 & & & 54 & & & \\
\hline $6-2$ & 49.65 & 127 & & & & & & \\
\hline $6-3$ & 49.78 & & & & & 207 & 83 & 0.31 \\
\hline $6-3$ & 49.87 & 119 & 1.48 & & & & & \\
\hline $6-3$ & 49.93 & & & & 29.69 & & & \\
\hline $6-3$ & 49.94 & 121 & & & & & & \\
\hline $6-3$ & 50.00 & & & 1503 & & & & \\
\hline $6-3$ & 50.07 & 112 & & & 52 & & & \\
\hline $6-3$ & 50.26 & 120 & & & 26.95 & & & \\
\hline $6-3$ & 50.31 & & & 1493 & & & & \\
\hline $6-3$ & 50.34 & 110 & 1.43 & & 44 & & & \\
\hline 6-3 & 50.70 & 122 & & & 24.01 & & & \\
\hline $6-3$ & 50.78 & & & 1493 & & & & \\
\hline $6-3$ & 50.86 & 114 & 1.4 & & 26 & & & \\
\hline $\begin{array}{l}6-3 \\
6-3\end{array}$ & $\begin{array}{l}50.95 \\
50.99\end{array}$ & 118 & & 1490 & 19.31 & & & \\
\hline $\begin{array}{l}6-3 \\
6-3\end{array}$ & 51.09 & 109 & 1.43 & & $\begin{array}{l}19.31 \\
26\end{array}$ & & & \\
\hline $6-4$ & 51.24 & 121 & & & 35.97 & & & \\
\hline $6-4$ & 51.33 & 111 & 1.44 & & 65 & & & \\
\hline $6-4$ & 51.36 & 120 & 1.43 & & & 206 & 96 & 0.22 \\
\hline $6-4$ & 52.23 & 113 & 1.47 & 1490 & & & & \\
\hline $6-4$ & 52.29 & 116 & & & 46.35 & & & \\
\hline $6-4$ & 52.42 & & & 1506 & & & & \\
\hline $6-4$ & 52.49 & 109 & 1.43 & & 105 & & & \\
\hline $6-5$ & 52.73 & 113 & & & 44 & & & \\
\hline $6-5$ & 52.76 & & & 1499 & & & & \\
\hline $6-5$ & 52.84 & 110 & 1.43 & & 108 & & & \\
\hline $6-5$ & 53.05 & 122 & & & & & & \\
\hline $\begin{array}{l}6-5 \\
6-5\end{array}$ & $\begin{array}{l}53.09 \\
53.10\end{array}$ & 130 & & & & 194 & 86 & 0.35 \\
\hline $6-5$ & 53.38 & & & & & & & \\
\hline $6-5$ & 53.65 & & & 1564 & & & & \\
\hline $6-5$ & 53.68 & & & & & & & \\
\hline $6-5$ & 53.69 & 120 & & & & 186 & 84 & 0.35 \\
\hline $6-5$ & 53.82 & 102 & 1.48 & 1502 & & & & \\
\hline $6-5$ & 53.87 & 46 & & & 55.96 & & & \\
\hline $6-5$ & 53.99 & 102 & 1.45 & & & & & \\
\hline $6-6$ & 54.22 & 48 & 1.78 & & & 59 & 29 & 0.63 \\
\hline $6-6$ & 54.30 & 50 & & & 36.26 & & & \\
\hline 6-6 & 54.32 & & & 1504 & & & & \\
\hline 6-6 & 54.39 & 47 & 1.77 & & & & & \\
\hline 6-6 & 54.58 & 121 & & & & 72 & 31 & 213 \\
\hline $\begin{array}{l}6-6 \\
6-6\end{array}$ & $\begin{array}{l}54.62 \\
55.57\end{array}$ & 66 & 1.7 & 1515 & & 12 & 31 & 2.13 \\
\hline $6-6$ & 55.60 & 62 & & 1543 & & & & \\
\hline $6-6$ & 55.64 & & & & 45.96 & & & \\
\hline $6-6$ & 55.69 & 84 & 1.53 & & & & & \\
\hline $6-7$ & 55.94 & 119 & 1.43 & & & & & \\
\hline $6-7$ & 55.97 & 118 & 1.48 & 1506 & & & & \\
\hline $6-7$ & 56.05 & & & & 22.15 & & & \\
\hline $6-7$ & 56.12 & 54 & & & & & & \\
\hline $6-7$ & 56.17 & & & 1524 & 42.83 & & & \\
\hline $7-1$ & 56.21 & 127 & 1.39 & & & & & \\
\hline $7-1$ & 56.44 & 93 & 1.49 & & 13 & & & \\
\hline $7-1$ & 56.65 & & & & 11.37 & & & \\
\hline $7-1$ & 56.69 & 86 & 1.53 & & 4.8 & & & \\
\hline $7-1$ & 56.97 & 65 & & & & & & \\
\hline $7-1$ & 57.08 & 57 & & & & & & \\
\hline $\begin{array}{l}7-1 \\
7-1\end{array}$ & $\begin{array}{l}57.10 \\
57.32\end{array}$ & 55 & 1.78 & 1510 & & & & \\
\hline $7-1$ & 57.59 & 53 & 1.74 & & 5.59 & & & \\
\hline 7.2 & 57.74 & 56 & & & 8.53 & & & \\
\hline $7-2$ & 57.86 & & & 1525 & & & & \\
\hline $7-2$ & 57.94 & 56 & 1.74 & & 4.02 & & & \\
\hline $7-2$ & 58.72 & 51 & 1.74 & & & 54 & 31 & 0.87 \\
\hline 7.2 & 59.02 & 52 & 1.79 & 1515 & & & & \\
\hline
\end{tabular}


Table 3. (Continued).

\begin{tabular}{|c|c|c|c|c|c|c|c|c|}
\hline Core-Section & $\begin{array}{l}\text { Sub-bottom } \\
\text { depth } \\
\text { (m) }\end{array}$ & $\begin{array}{c}\text { Water } \\
\text { content } \\
(\%)\end{array}$ & $\begin{array}{c}\text { Bulk } \\
\text { density } \\
\left(\mathrm{Mg} / \mathrm{m}^{3}\right)\end{array}$ & $\begin{array}{l}\text { Compress. } \\
\text { velocity } \\
(\mathrm{m} / \mathrm{s})\end{array}$ & $\begin{array}{c}\text { Shear } \\
\text { strength } \\
(\mathrm{kPa})\end{array}$ & $\begin{array}{l}\text { Liquid } \\
\text { limit } \\
(\%)\end{array}$ & $\begin{array}{c}\text { Plastic } \\
\text { limit } \\
(\%)\end{array}$ & $\begin{array}{l}\text { Liquidity } \\
\text { index }\end{array}$ \\
\hline $7-2$ & 59.04 & 53 & & & 5.29 & & & \\
\hline $7-2$ & 59.13 & & & 1546 & & & & \\
\hline $7-2$ & 59.19 & 49 & 1.76 & & 7.45 & & & \\
\hline $7-3$ & 59.30 & 49 & & & 19.5 & & & \\
\hline $7-3$ & 59.32 & & & 1553 & & & & \\
\hline $7-3$ & 59.39 & 52 & 1.73 & & 5.29 & & & \\
\hline $7-3$ & 59.57 & 52 & 1.78 & 1518 & & & & \\
\hline $7-3$ & 59.61 & & & 1538 & & & & \\
\hline $7-3$ & 59.65 & & & & 11.17 & & & \\
\hline $7-3$ & 59.79 & 51 & 1.76 & & 5.59 & & & \\
\hline $7-3$ & 59.98 & 53 & & & & & & \\
\hline $7-3$ & 60.03 & 71 & & & & & & \\
\hline $7-3$ & 60.09 & 72 & & & & & & \\
\hline $7-3$ & 60.54 & & & & & & & \\
\hline $7-3$ & 60.60 & & & & & & & \\
\hline $7-4$ & 60.70 & 56 & 1.71 & & & & & \\
\hline $7-4$ & 61.15 & 74 & 1.84 & 1528 & 14.7 & & & \\
\hline $7-4$ & 61.17 & 51 & & & & & & \\
\hline $7-4$ & 61.20 & & & 1546 & & & & \\
\hline $7-4$ & 61.24 & 53 & 1.73 & & 8.82 & & & \\
\hline $7-4$ & 61.40 & & & & & & & \\
\hline $7-4$ & 61.53 & & & & & 53 & 31 & 0.99 \\
\hline $7-4$ & 61.59 & 53 & & & & & & \\
\hline $7-4$ & 61.76 & 53 & 1.71 & & 17 & & & \\
\hline $7-4$ & 61.99 & & & & 86 & & & \\
\hline $7-5$ & 62.23 & & & & & & & \\
\hline $7-5$ & 62.43 & 54 & & & & & & \\
\hline $7-5$ & 62.50 & & & & & & & \\
\hline $7-5$ & 62.55 & & & & & & & \\
\hline $7-5$ & 62.63 & 121 & & & 9.02 & & & \\
\hline $7-5$ & 62.70 & & & & & 56 & 28 & 1 \\
\hline $7-5$ & 62.78 & 54 & & 1539 & & & & \\
\hline $7-5$ & 62.83 & & & & & 49 & & 1.15 \\
\hline $7-5$ & 62.84 & 52 & 1.74 & & 5.88 & & & \\
\hline $7-5$ & 63.02 & 53 & & & & & & \\
\hline $7-5$ & 63.08 & & & & & 40 & 30 & 2.31 \\
\hline $7-5$ & 63.17 & & & & & & & \\
\hline $7-5$ & 63.18 & 52 & & & & 45 & 32 & 1.51 \\
\hline $7-5$ & 63.41 & & & 1518 & & & & \\
\hline $7-5$ & 63.47 & 46 & & & 17.64 & & & \\
\hline $7-5$ & 63.53 & & & 1546 & & & & \\
\hline $7-5$ & 63.59 & 48 & 1.75 & & 9.31 & & & \\
\hline $7-5$ & 63.61 & 51 & 1.72 & & & & & \\
\hline $7-6$ & 63.87 & 51 & 1.8 & 1522 & & & & \\
\hline $7-6$ & 63.91 & 50 & & & 17.84 & & & \\
\hline $7-6$ & 68.95 & & & 1544 & & & & \\
\hline $7-6$ & 63.99 & 49 & 1.8 & & 7.74 & & & \\
\hline $7-6$ & 64.22 & 113 & & & & & & \\
\hline $7-6$ & 64.28 & & & & & 240 & 88 & 0.13 \\
\hline $7-6$ & 64.84 & 51 & 1.76 & & 4.02 & & & \\
\hline $7-6$ & 64.87 & 53 & 1.78 & 1520 & & & & \\
\hline $7-6$ & 65.09 & 46 & 1.79 & & 8.23 & & & \\
\hline $7-7$ & 65.25 & 49 & & & 11.96 & & & \\
\hline $7-7$ & 65.34 & & & 1546 & & & & \\
\hline $7-7$ & 65.39 & 47 & 1.79 & & 6.37 & & & \\
\hline $7-7$ & 65.75 & & & 1516 & 70.66 & & & \\
\hline $7-7$ & 65.89 & 51 & 1.66 & & 129 & & & \\
\hline
\end{tabular}

Results from Unit II (55-64 m sub-bottom) reflect the lithologic change from predominantly pelagic clays to carbonate ooze interbedded with pelagic clays. Water content and activity values for the carbonates are the lowest observed in Hole 576A; whereas bulk density and compressional velocity values are the highest observed. Atterberg limit data show low plasticity and liquidity indices in the carbonates.

\section{CONSOLIDATION AND PERMEABILITY CHARACTERISTICS}

Skempton (1970) defined consolidation as the result of all processes causing the progressive transformation of an argillaceous sediment from a soft clay to a shale. These processes include: (1) interparticle bonding, (2) dessication, (3) cementation, and (4) the squeezing out of pore water under the increasing weight of overburden. The effect of each process on consolidation varies through time.

Consolidation tests are used to evaluate the sediment response to an applied load. These results provide insight to the relative degree of consolidation the sediment has experienced in situ under the imposed load of the sedimentary column. The state of consolidation is determined using the ratio of preconsolidation stress $\left(\sigma_{\mathrm{c}}\right)$ to the present effective overburden stress $\left(\sigma_{\mathrm{o}}^{\prime}\right)$. Preconsoli- 


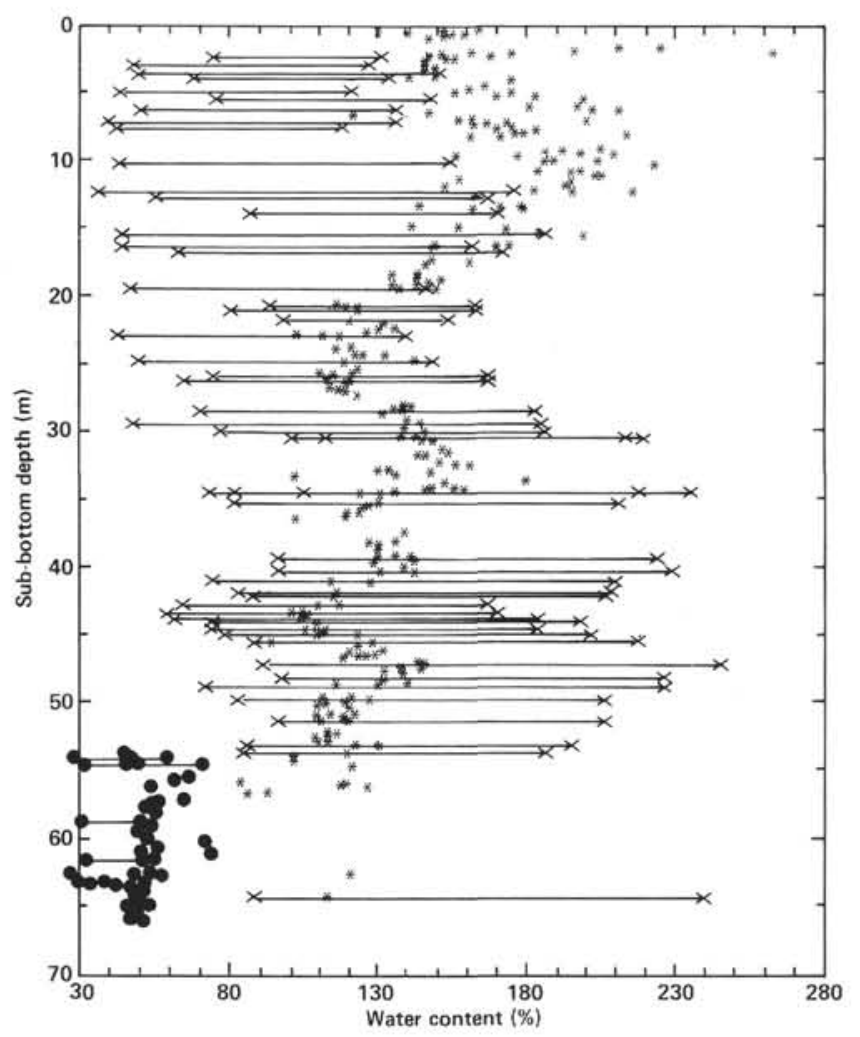

Figure 7. Atterberg limit and water-content results obtained from Hole 576A sediments. Liquid and plastic limit values fall on the right and left side of the profile, respectively. Solid circles ( $\bullet$ ) below $55 \mathrm{~m}$ represent values obtained in carbonate ooze.

dation stress, defined as the maximum effective stress the sediment has experienced, was calculated using the graphical reconstruction technique of Casagrande (1936). The value of $\sigma_{\mathrm{o}}^{\prime}$ is calculated assuming hydrostatic conditions. The ratio of the preconsolidation stress to the effective overburden stress yields the overconsolidation ratio $\left(\mathrm{OCR}=\sigma_{\mathrm{c}}^{\prime} / \sigma_{\mathrm{o}}^{\prime}\right)$.

A sediment is considered normally consolidated if the present effective overburden stress is the greatest ever imposed. Thus, for a normally consolidated sediment the preconsolidation stress equals or closely approximates the effective overburden stress $\left(\sigma_{\mathrm{c}}^{\prime}=\sigma_{\mathrm{o}}^{\prime}\right)$. A sediment is overconsolidated if it has been consolidated under a stress that exceeds the present effective overburden stress $\left(\sigma_{\mathrm{c}}^{\prime}\right.$ $\left.>\sigma_{\mathrm{o}}^{\prime}\right)$. Sediment that has not fully consolidated under the present overburden stress is underconsolidated. For underconsolidated sediments the pore-water pressure exceeds the hydrostatic pressure and the preconsolidation stress will be less than the effective overburden stress ( $\sigma_{\mathrm{c}}^{\prime}$ $<\sigma_{\mathrm{o}}^{\prime}$ ). Thus, sediments with overconsolidation ratios of approximately 1.0 are considered normally consolidated, greater than 1.0 are overconsolidated, and less than 1.0 are underconsolidated.

\section{Consolidation Test Results}

Consolidation test results from Hole 576A sediments probably represent the most extensive suite of consolidation data for any marine sedimentary section ( 80 con-

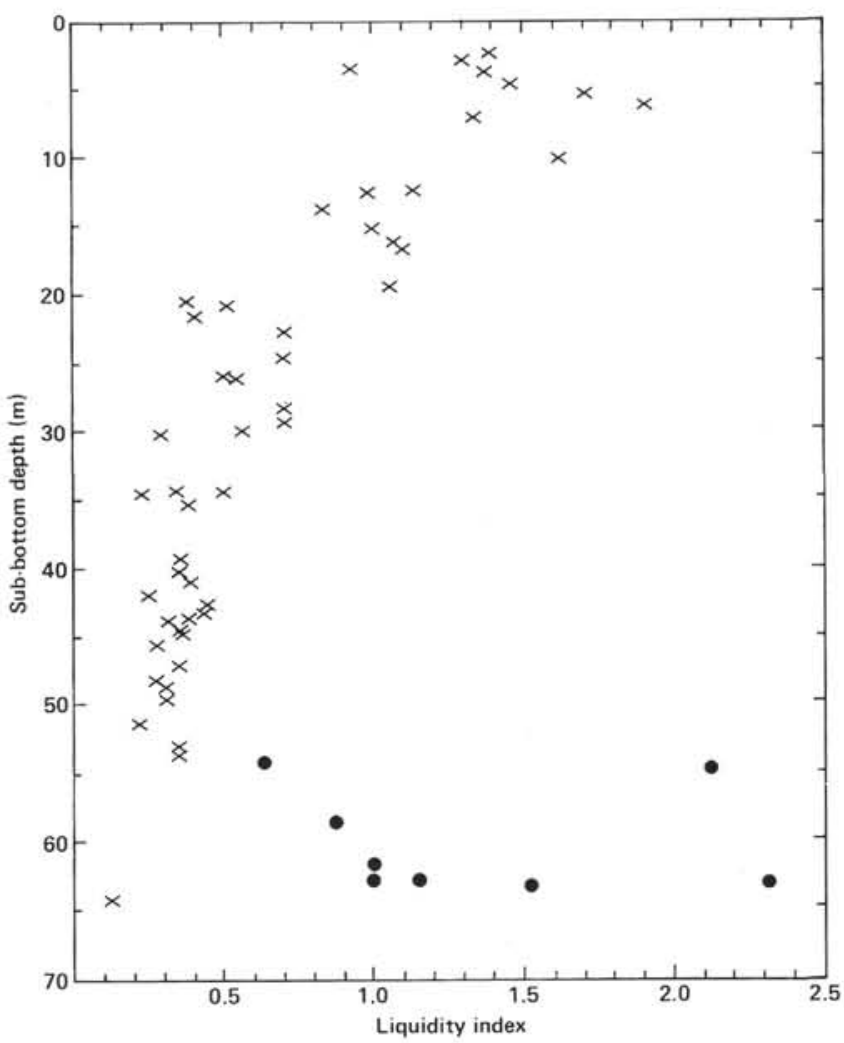

Figure 8. Liquidity index-depth profile for Hole 576A sediments. Values greater than 1.0 indicate material with natural water contents greater than the liquid limit. Solid circles (॰) below $55 \mathrm{~m}$ represent values obtained in carbonate ooze.

solidation tests performed on $63.70 \mathrm{~m}$ of sediment; $\simeq 1$ test every $0.80 \mathrm{~m}$ ). This extensive testing program was initiated because the geological history of the area near Site 576 is well-understood and the principles of consolidation theory suggested that such data may be used to define a model of the consolidation process for "a perfect marine clay section" that has been continuously depositional over the last 65 m.y. (Heath, Rea and Levi, this volume). All geological and geotechnical information available prior to beginning the testing program indicated that these sediments would be normally consolidated below the upper few meters. However, test results indicate that the sediments at Hole 576A are overconsolidated in the upper $28 \mathrm{~m}$, normally consolidated in the interval between 28 and $39 \mathrm{~m}$ sub-bottom, and normally to underconsolidated in the interval between 39 and $55 \mathrm{~m}$ sub-bottom (Fig. 15; Tables 4 and 5). The carbonate-ooze consolidation test results are included in the data compilation for comparison with the pelagic clay results even though these results are not emphasized in the following discussion because of their differences in geotechnical behavior and mode of deposition.

Typical $e$ versus $\log \sigma^{\prime}$ curves for tests performed in both Subunit IA (0-28 m sub-bottom) and Subunit IB (28-55 m sub-bottom) reveal significant differences in initial void ratios and the sediment response to both an applied load and an unloading sequence (Fig. 16). These differences are also reflected in the average values of several consolidation parameters and the permeability val- 


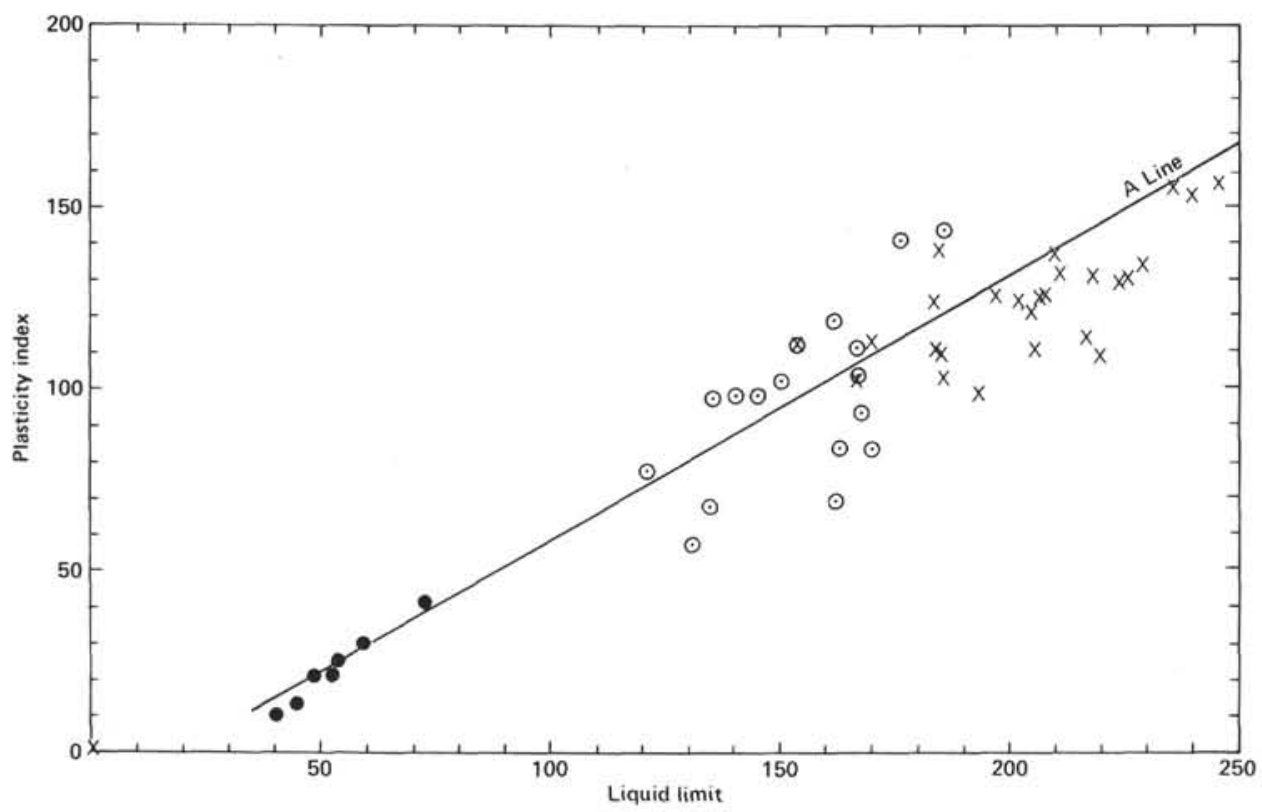

Figure 9. Standard plasticity chart used for classifying fine-grained sediments (Casagrande, 1948). Casagande's A line is shown. Subunit IB sediments (X) are generally much more plastic than Subunit IA sediments $(\odot)$ or the carbonate oozes $(\bullet)$.

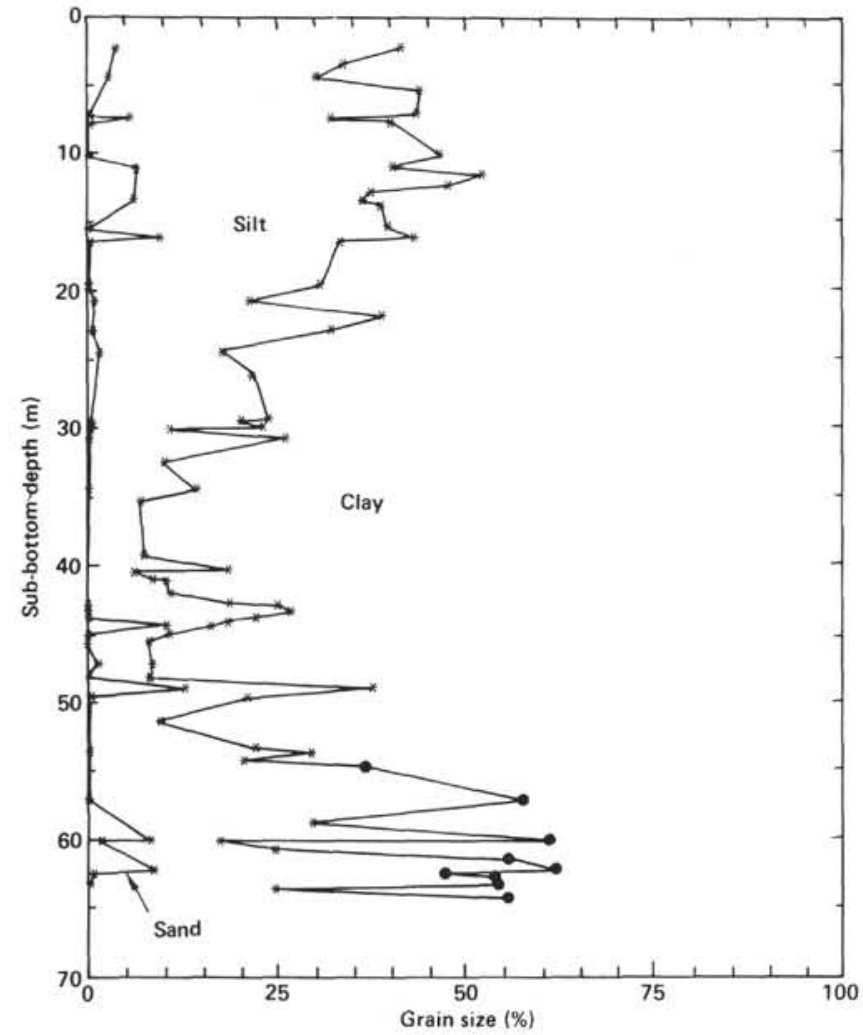

Figure 10. Grain size-depth profile for Hole 576A sediments. Solid circles $(\bullet)$ below $55 \mathrm{~m}$ represent values obtained in carbonate ooze.

ues reported for each of the major lithologic units (Tables 4 and 5). A compilation of the consolidation test results is illustrated in Figure 17, which shows the void ratios at applied loads of $50,100,300$, and $1000 \mathrm{kPa}$ plotted versus sub-bottom depth.

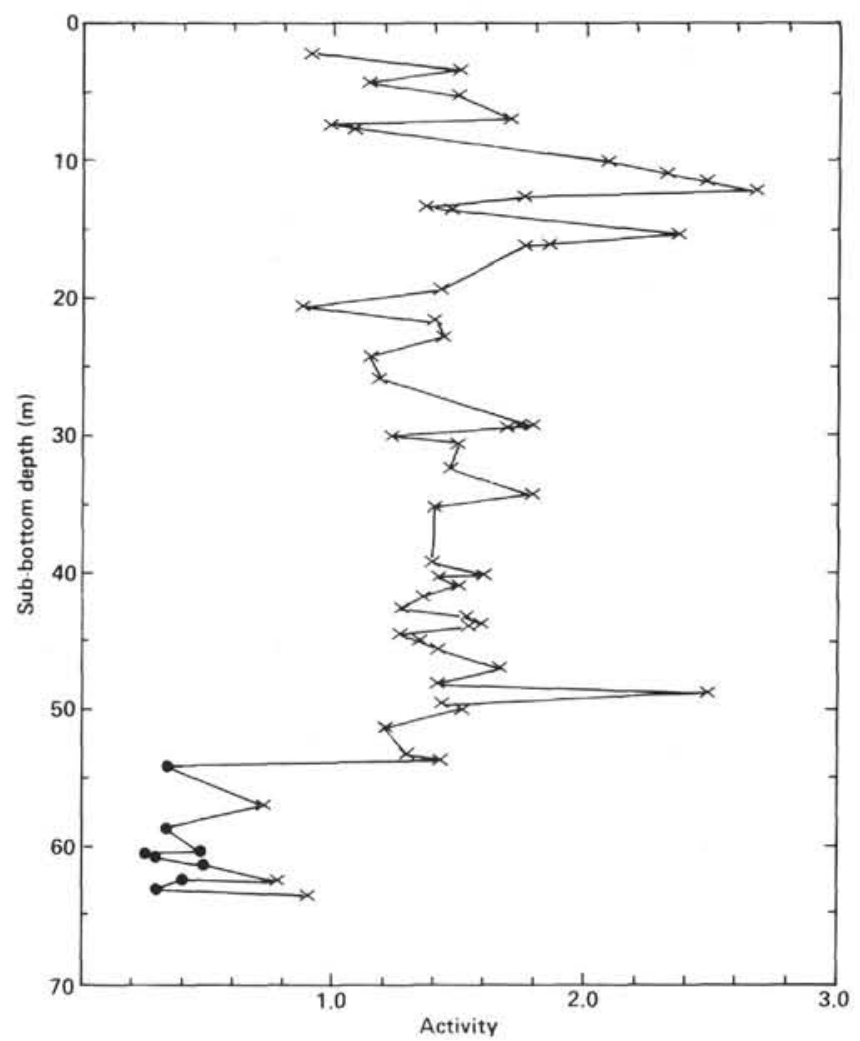

Figure 11. Activity-depth profile for Hole 576A sediments. Solid circles ( $\bullet$ ) below $55 \mathrm{~m}$ represent values obtained in carbonate ooze.

The overconsolidated surficial sediments from Hole $576 \mathrm{~A}$ were not totally unexpected. Consolidation test results from many different marine geological environments indicate that most near-surface sediments are in a state of 'apparent overconsolidation' (Hamilton, 1964; She- 


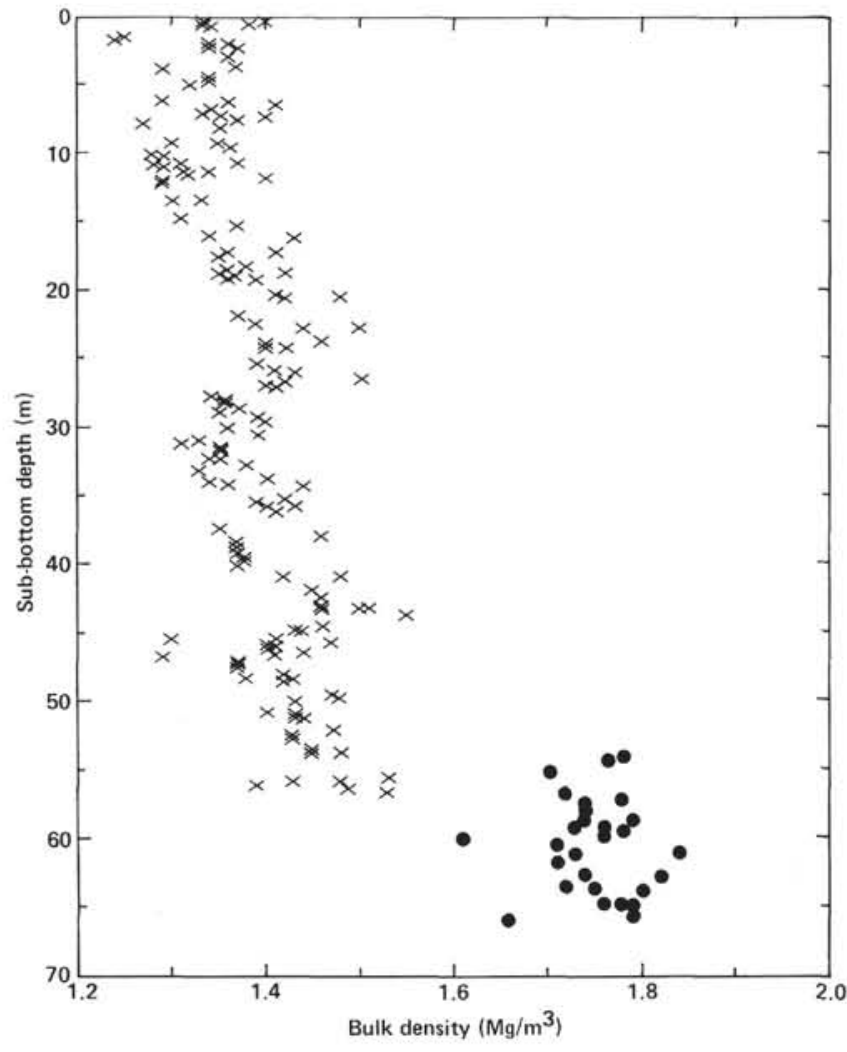

Figure 12. Bulk density-depth profile for Hole 576A sediments. Solid circles $(\bullet)$ below $55 \mathrm{~m}$ represent values obtained in carbonate ooze.

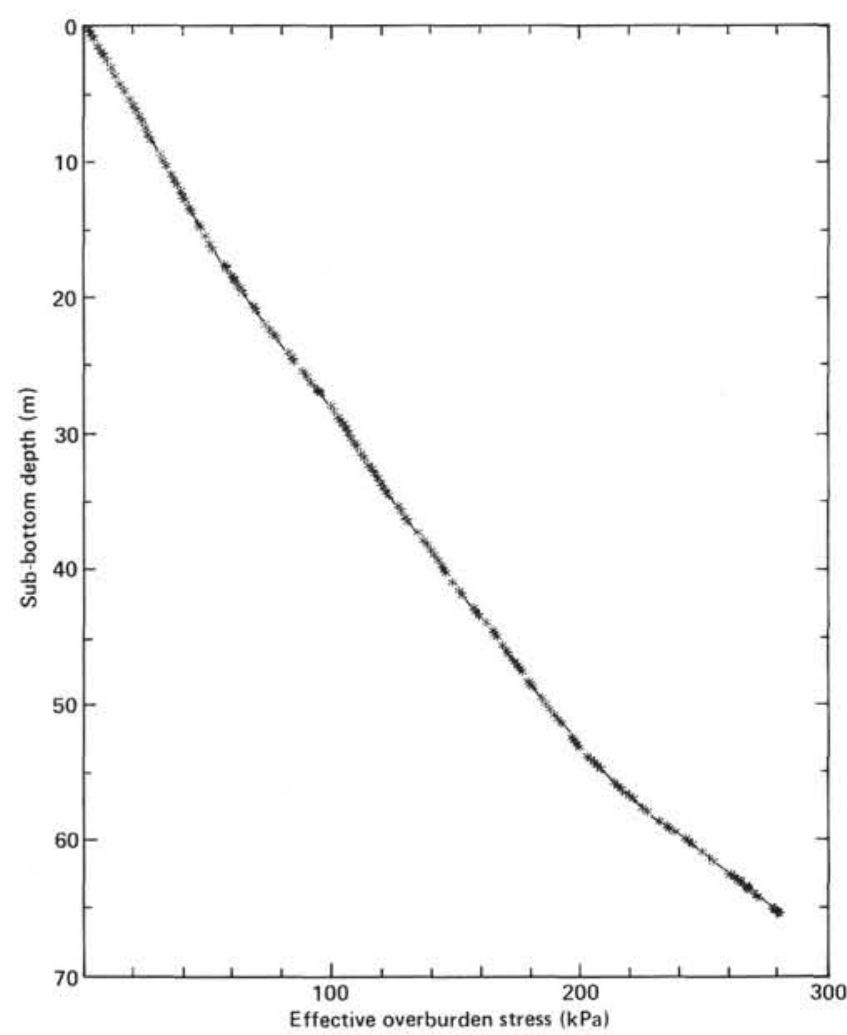

Figure 13. Effective overburden stress-depth profile computed using bulk-density values for Hole 576A sediments. Effective overburden stress was calculated assuming hydrostatic pore-water pressure conditions using the equation of Shephard et al. (1978).

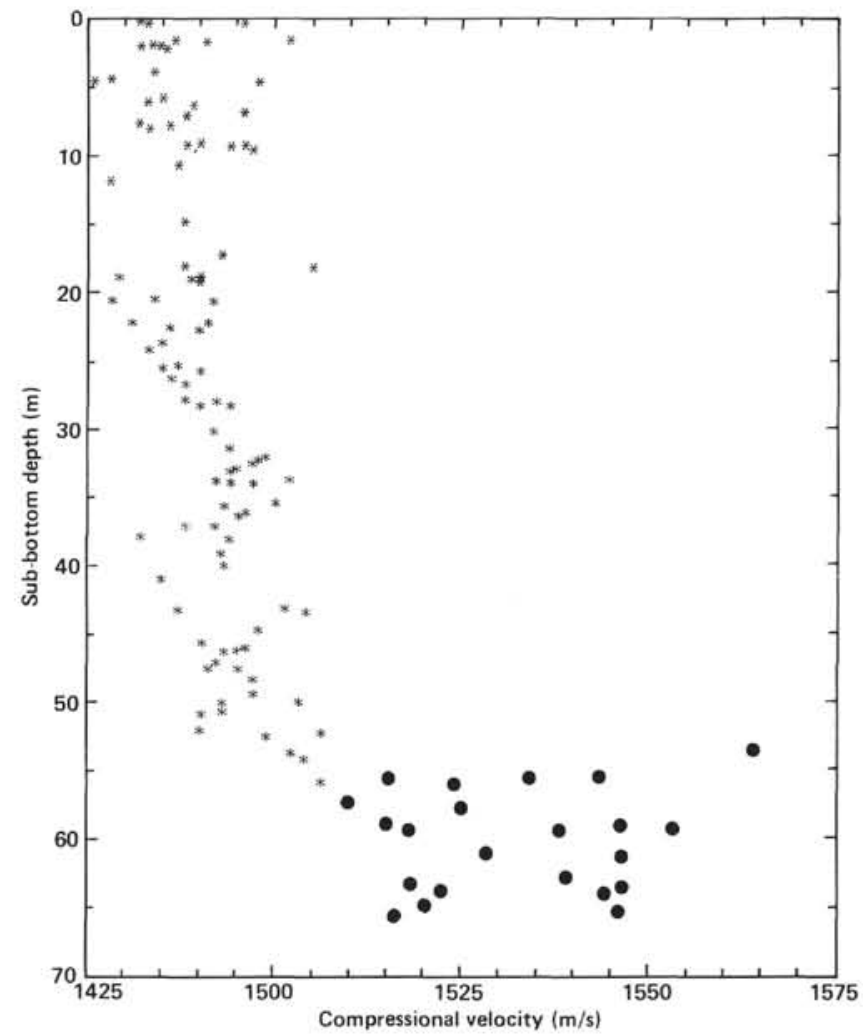

Figure 14. Compressional velocity-depth profile for Hole 576A sediments. Solid circles $(\bullet)$ below $55 \mathrm{~m}$ represent values obtained in carbonate ooze.

phard et al., 1978; Silva et al., 1980). Apparent overconsolidation is generally attributed to the cohesion that results from the molecular interaction between solid particles (Terzaghi, 1956). For clay sediments, Terzaghi suggested these cohesive forces are at least $5 \mathrm{kPa}$. In nearsurface sediments these forces are large relative to the small overburden stress, resulting in a calculated preconsolidation stress value that is much larger than the effective overburden stress and, consequently, an overconsolidation ratio that is significantly greater than unity.

A number of mechanisms or processes can influence the state of consolidation (Brumund et al., 1976). The most probable mechanisms responsible for the overconsolidation of the Subunit IA sediments are (1) changes in soil structure due to chemical alterations in the form of cementing agents and interparticle bonds, (2) the effects of high hydrostatic pressure release during core recovery, and (3) artifacts arising from the consolidation test process. Dadey (1983) assumed that the effects of cementation and interparticle bonding on the sediment microstructure would be relatively constant with depth; thus, its significance would be gradually diminished with increasing overburden. Consequently, as is evident in Figure 15, the overconsolidation ratio will decrease with depth, eventually reaching a value that fluctuates around one. This occurs at approximately 25 to $30 \mathrm{~m}$ sub-bottom in Hole 576A sediments.

Brumund and Callender (1975) concluded that the apparent overconsolidation present in near-surface sediments was related to the release of high hydrostatic pressures during sampling. Lee (1984) however, suggests that 


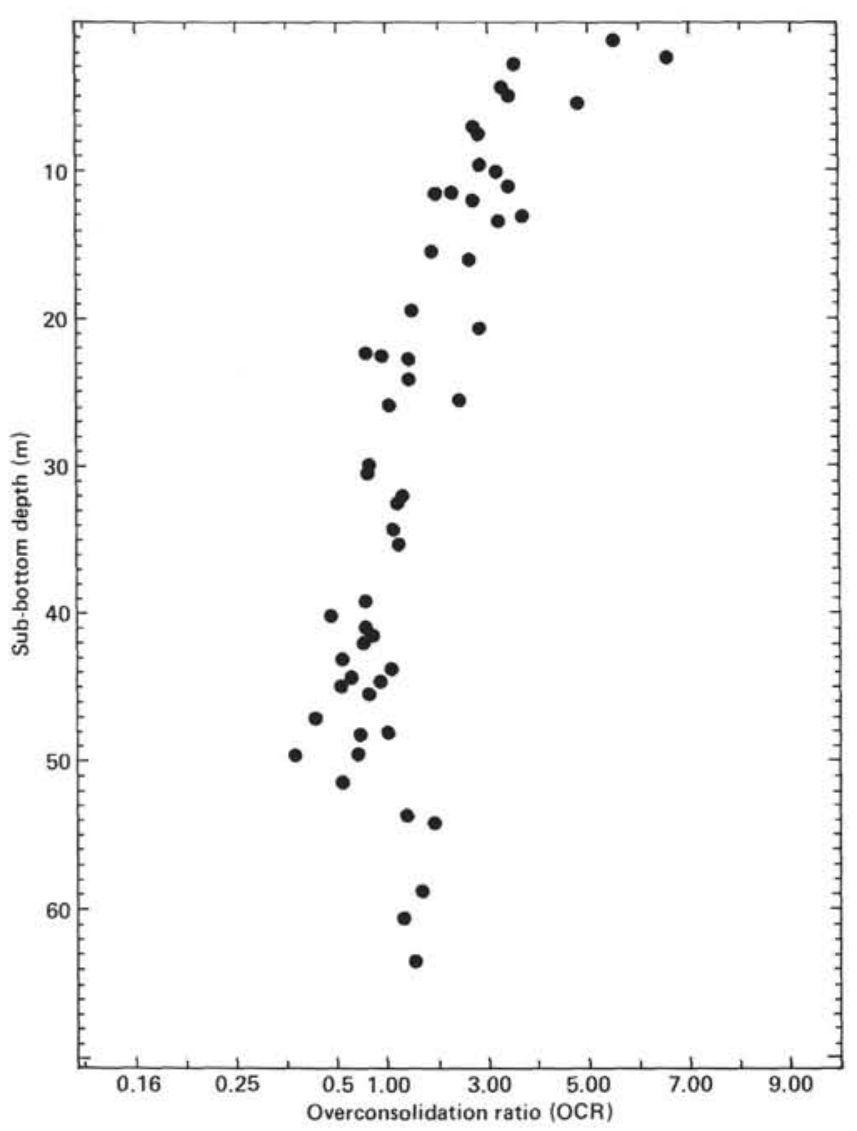

Figure 15. Overconsolidation ratio-depth profile computed using static-load consolidation test results from Hole 576A sediments. Overconsolidation scale reciprocal below 1.00, linear above 1.00 .

clays exposed to hydrostatic pressures of 75 to $100 \mathrm{MPa}$ would have an induced effective stress of only 2 to $5 \mathrm{kPa}$. This is not of sufficient magnitude to affect significantly the preconsolidation stress values determined using the Casagrande method for Hole 576A sediments.

Scully et al. (1984) have shown that completely remolded, normally consolidated sediments develop a fictitious preconsolidation stress, which they attribute to seating of the porous stones and loading platens during initial testing and possibly to wall fraction developed during the consolidation test. This artificial preconsolidation stress is of uncertain magnitude, but may contribute to the apparent overconsolidation observed in Hole 576 A sediments.

The sediments between 28 and $39 \mathrm{~m}$ sub-bottom appear normally consolidated while those between 38 and $55 \mathrm{~m}$ sub-bottom appear normally to underconsolidated. In fact, OCR results indicate that in some instances the sediments between 39 and $55 \mathrm{~m}$ sub-bottom are as underconsolidated as the sediments between the seafloor and $28 \mathrm{~m}$ sub-bottom are overconsolidated (Fig. 15). The mechanisms generally responsible for underconsolidation include high rates of sediment accumulation, chemical alterations, and coring disturbance (Sangrey, 1977). Sediment accumulation rates at Hole 576A are generally much less than $10 \mathrm{~m} / \mathrm{m}$.y. This rate is sufficiently slow to permit the dissipation of any excess pore water pressures that may have developed during the sedimentation process. Available geochemical data for Site 576A (McDuff, this volume) suggest that any chemical alteration effects should be prevalent within all of Subunit IB and not limited to only select intervals. Although coring and sampling disturbance may have contributed to the observed state of underconsolidation, this effect is very difficult to quantify.

The discrepancy between the expected normal state of consolidation and the consolidation test results strongly indicates that the technique employed for determining the preconsolidation stress (Casagrande, 1936) should be carefully evaluated. Various investigators, including Cooling and Skempton (1942), Bishop et al. (1965), and Schmertmann (1955), have concluded that the Casagrande method is inadequate to define the preconsolidation stress under certain circumstances. Other methods used to determine the preconsolidation stress (Burmister, 1951; Schmertmann, 1955) require a rebound and reloading cycle.

Examination of the $e$ versus $\log \sigma^{\prime}$ curves (Fig. 16) and the profile of expansion index versus sub-bottom depth (Fig. 18) shows that the amount of sediment expansion or rebound that occurs during unloading increases with depth below the seafloor. As the expansion index increases, the error in the determination of the preconsolidation stress, as determined by the Casagrande method, also appears to increase. An examination of the unloading and reloading portions of the $e$ versus $\log \sigma^{\prime}$ curve at $32.47 \mathrm{~m}$ sub-bottom (Fig. 16) shows that the unloading portion of the hysteresis loop in this example started at $400 \mathrm{kPa}$. Thus, the actual preconsolidation stress of the hysteresis-loop curve is $400 \mathrm{kPa}$. However, when the preconsolidation stress is determined using the Casagrande method on the hysteresis loop the preconsolidation stress is underestimated by $140 \mathrm{kPa}(35 \%)$.

These results and the subsequent analysis of $16 e$ versus $\log \sigma^{\prime}$ curves containing a hysteresis loop have been used to develop a relationship between the expansion index and the preconsolidation stress as determined by the Casagrande method (Fig. 19). This relationship was used to adjust the values of the preconsolidation stress determined using the Casagrande method. The adjusted values are listed in Table 6 as $\sigma_{\mathrm{c} 4}$.

Another method to determine the preconsolidation stress is based on the characteristics of the rebound curve and the assumption that these characteristics are constant for a given sediment sample (Fig. 20). An examination of all the Site $576 \mathrm{~A} e$ versus $\log \sigma^{\prime}$ curves with rebound portions substantiates this assumption. The rebound method for determining the preconsolidation stress utilizes a point on the constant or maximum void ratio line that occurs prior to the maximum point of curvature on the $e$ versus $\log \sigma^{\prime}$ curve. This point (point B in Fig. 20) should be located at the maximum effective stress occurring on the line of constant void ratio. If that point appears ambiguous, a point one complete log cycle before the point of maximum curvature on the $e$ versus log $\sigma^{\prime}$ curve can be used. From that point, a line parallel to the rebound portion of the $e$ versus $\log \sigma^{\prime}$ curve is extended to intersect the virgin curve. That intersection is 
Table 4. Summary of incrementally loaded consolidation test results performed on Hole 576A sediments.

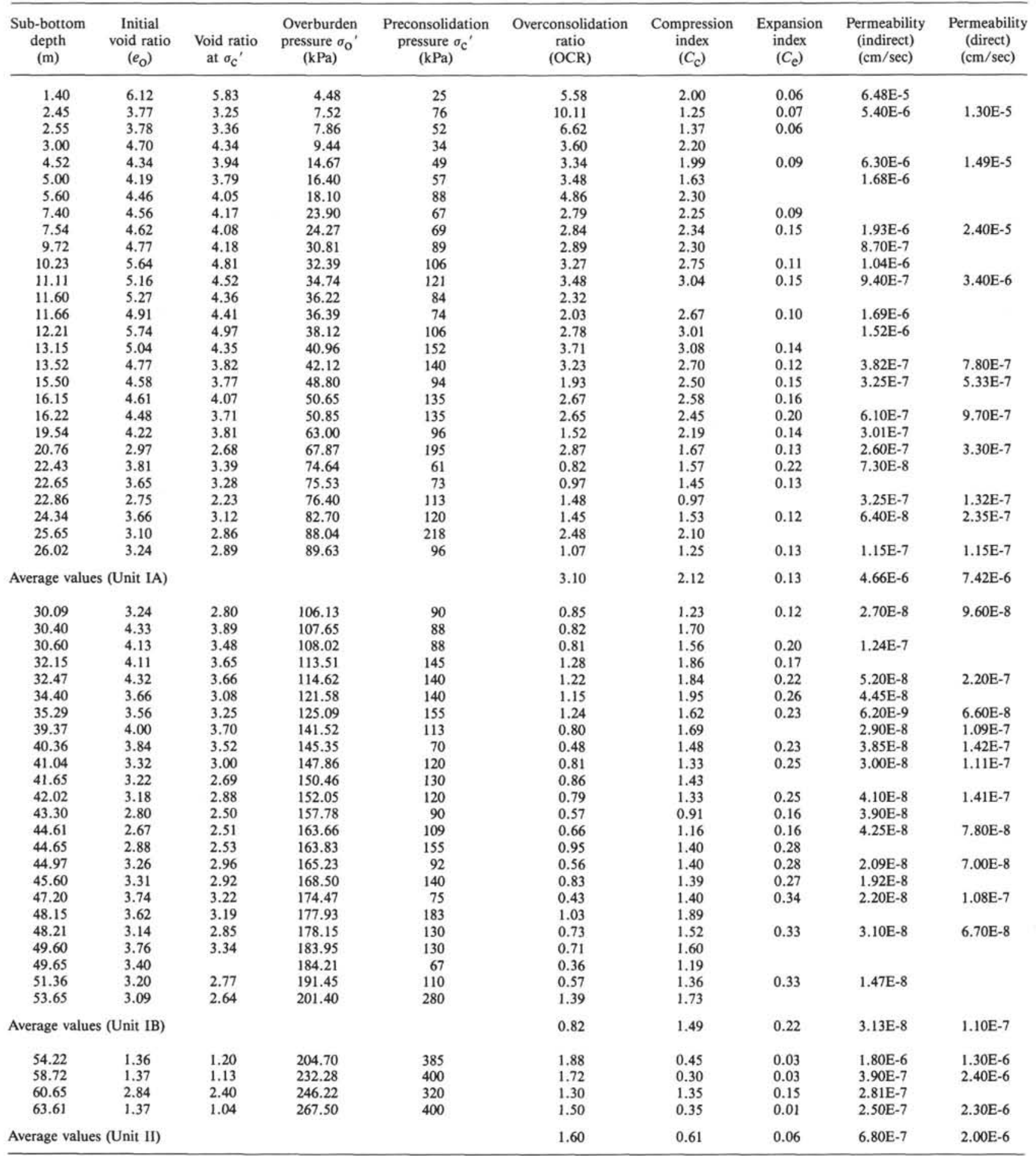

determined to be the preconsolidation stress. Values derived by this method are listed in Table 6 as $\sigma_{\mathrm{cs}}$.

The interpretation of the consolidation test results can vary significantly depending on the technique employed for determining the preconsolidation stress (Table 6). Results using the Casagrande technique $\left(\sigma_{\mathrm{c} 1}^{\prime}\right)$ indicate that Subunit IA sediments are overconsolidated and Subunit IB sediments are normally to underconsolidated. The second technique used for determining the preconsolida- tion stress $\left(\sigma_{c 4}^{\prime}\right)$ results in almost all Hole 576A sediments being overconsolidated. The third technique, which uses point $\mathrm{B}\left(\sigma_{\mathrm{c} s}^{\prime}\right)$, results in the upper sediment $(0-28 \mathrm{~m}$ subbottom) being, in essence, less overconsolidated than the results using $\sigma_{\mathrm{c} 1}$, and the lower sediment ( 28 to $55 \mathrm{~m}$ sub-bottom) normally consolidated. This third technique appears to reflect best the anticipated state of consolidation of Subunit IB based on the geological history of this area. 
Table 5. Summary of constant rate of deformation consolidation tests performed on Hole 576A sediments.

\begin{tabular}{|c|c|c|c|c|c|c|c|c|c|}
\hline $\begin{array}{l}\text { Sub-bottom } \\
\text { depth } \\
\text { (m) }\end{array}$ & $\begin{array}{c}\text { Initial } \\
\text { void ratio } \\
\left(e_{0}\right)\end{array}$ & $\begin{array}{l}\text { Void ratio } \\
\text { at } \sigma_{\mathrm{c}}^{\prime}\end{array}$ & $\begin{array}{l}\text { Overburden } \\
\text { pressure } \sigma_{o} \\
\quad(\mathrm{kPa})\end{array}$ & $\begin{array}{l}\text { Preconsolidation } \\
\text { pressure } \sigma_{\mathrm{c}}{ }^{\prime} \\
(\mathrm{kPa})\end{array}$ & $\begin{array}{c}\text { Overconsolidation } \\
\text { ratio } \\
\text { (OCR) }\end{array}$ & $\begin{array}{l}\text { Compression } \\
\text { index } \\
\left(C_{\mathrm{c}}\right)\end{array}$ & $\begin{array}{c}\text { Expansion } \\
\text { index } \\
\left(C_{\mathrm{e}}\right)\end{array}$ & $\begin{array}{l}\text { Permeability } \\
\text { (indirect) } \\
\text { (cm/sec) }\end{array}$ & $\begin{array}{c}\text { Permeability } \\
\text { (direct) } \\
(\mathrm{cm} / \mathrm{sec})\end{array}$ \\
\hline 4.60 & 3.58 & 4.12 & 14.93 & 69 & 4.62 & 1.71 & 0.15 & & $1.70 \mathrm{E}-7$ \\
\hline 7.60 & 4.05 & 3.58 & 24.46 & 100 & 4.09 & 2.02 & 0.14 & & $5.10 \mathrm{E}-7$ \\
\hline 11.20 & 4.74 & 4.02 & 35.01 & 105 & 3.00 & 2.42 & 0.26 & & $6.70 \mathrm{E}-7$ \\
\hline 13.60 & 4.40 & 3.62 & 42.26 & 120 & 2.84 & 2.04 & 0.07 & & $4.45 \mathrm{E}-7$ \\
\hline 16.30 & 4.49 & 3.55 & 51.13 & 160 & 3.13 & 2.70 & 0.02 & & $1.65 \mathrm{E}-7$ \\
\hline 20.85 & 3.01 & 2.44 & 68.60 & 179 & 2.61 & 2.11 & & $5.19 \mathrm{E}-8$ & \\
\hline 24.40 & 3.69 & 2.73 & 82.92 & 172 & 2.07 & 2.12 & & $4.85 \mathrm{E}-8$ & \\
\hline 26.10 & 3.38 & 2.49 & 89.97 & 215 & 2.40 & 2.10 & & $3.15 \mathrm{E}-8$ & \\
\hline 30.20 & 3.18 & 2.83 & 107.28 & 88 & 0.82 & 1.97 & & $5.35 \mathrm{E}-8$ & \\
\hline 32.55 & 3.99 & 3.55 & 114.91 & 118 & 1.03 & 2.56 & & $5.65 \mathrm{E}-8$ & \\
\hline 35.25 & 3.32 & 3.07 & 124.92 & 90 & 0.72 & 2.45 & & $4.50 \mathrm{E}-8$ & \\
\hline 39.45 & 3.21 & 2.81 & 141.83 & 100 & 0.70 & 2.23 & & $3.21 \mathrm{E}-8$ & \\
\hline 40.44 & 4.19 & 3.73 & 145.63 & 48 & 0.33 & 1.73 & 0.40 & & $7.20 \mathrm{E}-8$ \\
\hline 41.04 & 3.07 & 2.62 & 147.90 & 120 & 0.81 & 1.15 & & $3.95 \mathrm{E}-8$ & \\
\hline 42.10 & 2.92 & 2.61 & 152.40 & 115 & 0.75 & 1.86 & & $5.40 \mathrm{E}-8$ & \\
\hline 43.70 & 2.57 & 2.26 & 159.89 & 115 & 0.72 & 1.76 & & $3.55 \mathrm{E}-8$ & \\
\hline 45.10 & 2.97 & 2.81 & 165.84 & 65 & 0.39 & 1.83 & & $4.60 \mathrm{E}-8$ & \\
\hline 45.70 & 3.10 & 2.88 & 168.81 & 88 & 0.52 & 2.16 & & $4.50 \mathrm{E}-8$ & \\
\hline 49.30 & 2.97 & 2.59 & 182.67 & 166 & 0.91 & 2.25 & & $2.36 \mathrm{E}-8$ & \\
\hline 52.40 & 2.99 & 2.70 & 203.28 & 118 & 0.58 & 2.09 & & $2.55 \mathrm{E}-8$ & \\
\hline 54.50 & 1.61 & 1.43 & 206.65 & 223 & 1.08 & 1.18 & & $1.55 \mathrm{E}-7$ & \\
\hline 58.80 & 1.27 & 0.86 & 232.87 & 675 & 2.90 & & & $1.83 \mathrm{E}-6$ & \\
\hline 61.70 & 1.16 & 0.80 & 253.89 & 700 & 2.76 & & & $3.05 \mathrm{E}-7$ & \\
\hline 63.70 & 1.08 & 0.75 & 270.35 & 700 & 2.59 & & & $6.30 \mathrm{E}-7$ & \\
\hline
\end{tabular}

The predicted field consolidation curve for Hole 576A pelagic sediments (Fig. 21) was constructed using the void ratio at the preconsolidation stress and the calculated in situ vertical effective stress. This curve can be used to interpolate in situ porosity-permeability-depth relationships for any portion of the sedimentary section. The quadratic line of best fit defines an $e$ versus $\log \sigma^{\prime}$ curve that has a compression index of 2.2 as compared to an average $C_{\mathrm{c}}$ of 2.12 for Subunit IA sediments (Table 4) and an initial void ratio of 5 to 5.4 .

\section{Permeability}

As indicated earlier, several means of measuring or calculating the coefficient of permeability were employed. All direct measurements were performed during the course of the consolidation test. The indirect determinations were calculated using either conventional consolidation theory or CRD test results. All reported permeability values were determined at the preconsolidation stress (Fig. 22). The permeability profile is characteristic of other results reported for fine-grained marine sediments and shows decreasing permeability with depth.

\section{Summary}

Detailed analysis of Hole 576A consolidation test results indicate that the accepted methodology for interpreting the geological significance of consolidation data is deficient. Sediments in the upper $28 \mathrm{~m}$ are overconsolidated regardless of how the preconsolidation stress is determined. Sediments between 28 and $39 \mathrm{~m}$ sub-bottom are normally consolidated; whereas sediments between 39 and $55 \mathrm{~m}$ sub-bottom appear normally to underconsolidated. A number of mechanisms have been proposed to explain the variation in consolidation behavior, although many of these mechanisms do not appear consistent with the well-substantiated geological history of this area. Much of the apparent discrepancy between the expected consolidation state of Hole 576A sediments based on available geological and geotechnical information and the actual consolidation test results may result from the Casagrande technique employed to determine the preconsolidation stress. An alternative method for calculating $\sigma_{\mathrm{c}}^{\prime}$, based on the characteristics of the rebound portion of the $e$ versus $\log \sigma^{\prime}$ curve, yields $\sigma_{\mathrm{c}}^{\prime}$ values for Subunit IB that are more consistent with the known geology in the area.

\section{SHEAR STRENGTH}

Shear strength tests were performed on Hole 576A sediments to determine the characteristics of the undrained shear strength profile with depth and to evaluate the significance of this profile relative to the observed consolidation state of Hole 576A sediments. The shear strength tests performed included: (1) laboratory vane shear; (2) unconsolidated, undrained triaxial compression; (3) isotropically consolidated, undrained triaxial compression; and (4) anistropically consolidated, undrained triaxial compression (Table 7). Results of these tests and their relationships to the observed geotechnical behavior of Hole 576A sediments are summarized in the following sections.

\section{Undrained Shear Strength}

The undrained shear strength of Hole 576A sediments was determined using the laboratory vane shear and the results from unconsolidated-undrained triaxial (UU) compression tests. Results of the vane shear tests are summarized in Figure 23 and Table 3. Table 8 summarizes the results of the UU triaxial tests. These data have been normalized using the ratio of the undrained shear strength to the effective overburden stress calculated for the subbottom depth at which the strength measurement was 

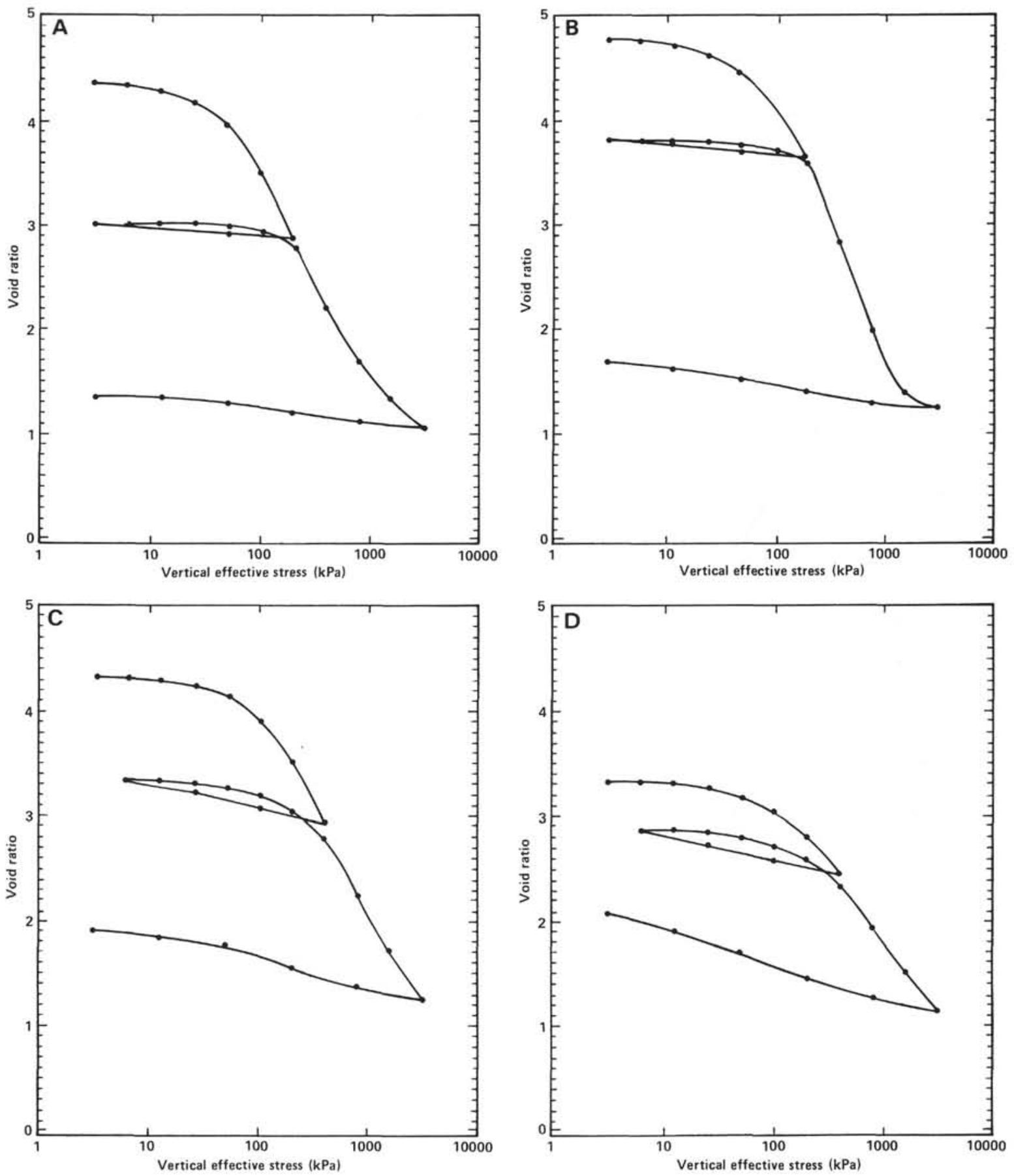

Figure 16. Void ratio versus $\log$ of effective stress curves ( $e$ versus $\left.\log \sigma^{\prime}\right)$ obtained from Hole 576A sediments located in Subunit IA (A, 4.20 and B, $13.52 \mathrm{~m}$ sub-bottom) and Subunit IB (C, 32.47 and D, $45.60 \mathrm{~m}$ sub-bottom).

made $\left(s_{\mathrm{u}} / \sigma_{\mathrm{o}}^{\prime}\right.$; Fig. 24$)$ for comparison to a computed in situ profile presented later. The values of $\sigma_{\circ}^{\prime}$ were obtained from Figure 13.

\section{Shear Strength Parameters}

Effective stress and undrained shear strength parameters were determined from consolidated-undrained triaxial tests for both isotropic and anistropic loading conditions. Table 7 lists the samples tested and the test conditions employed for each specimen. Examples of the stress paths and the effective stress parameters for both the isotropic and anisotropic condition are presented in Figures 25 and 26 with the corresponding shear-strength parameter values derived from the tests summarized in Tables 9 through 11 .

\section{Calculated Undrained Shear Strength Profiles}

The shear strength parameters measured on Hole 576A sediments provide a basis for calculating an in situ undrained shear strength profile. The calculations involve the use of the SHANSEP method (Ladd and Foott, 1976; Ladd et al., 1977), which relates the in situ normalized 


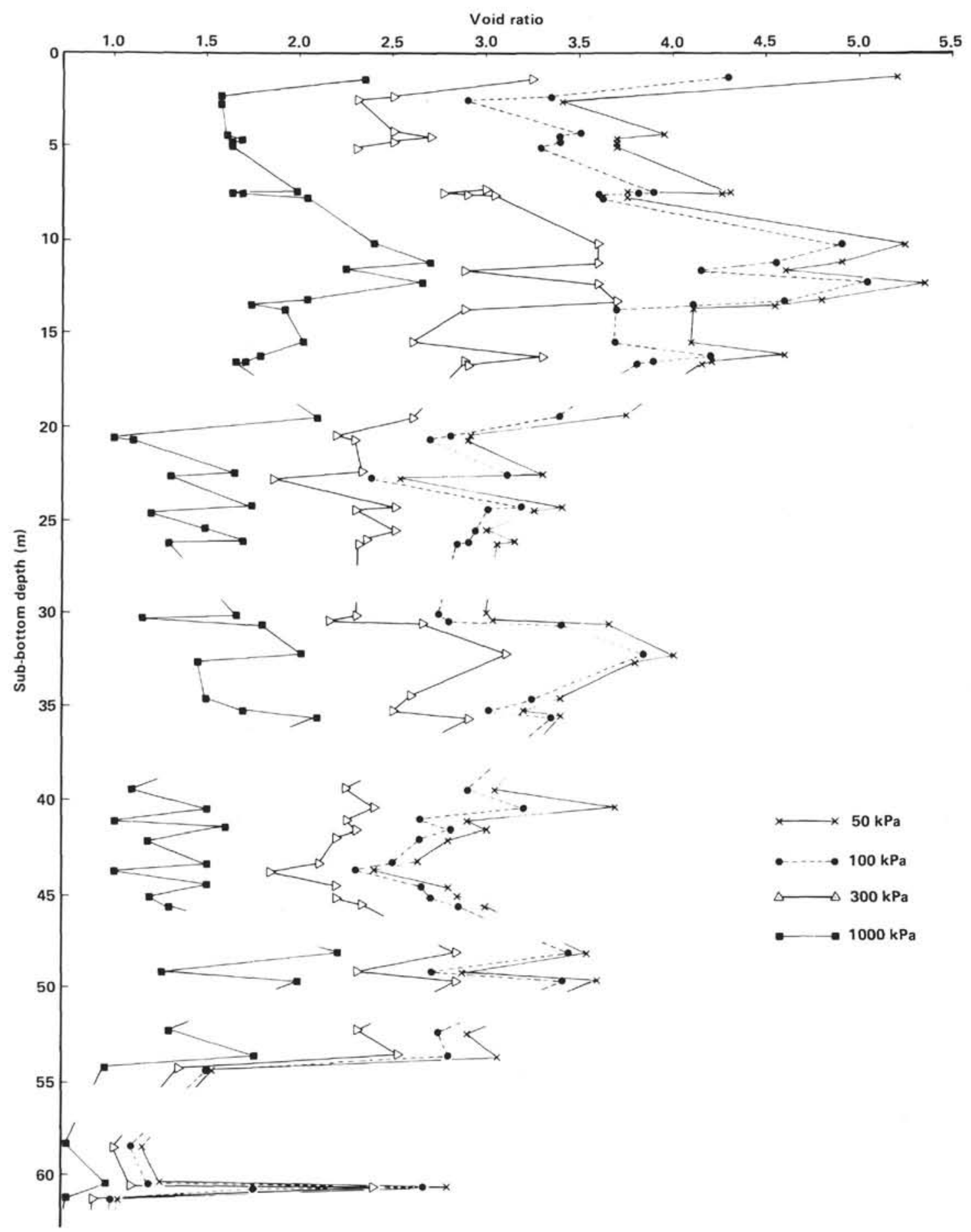

Figure 17. Summary of consolidation test results for Hole 576A sediments. Sediment response to increasing applied stress for each sample tested is displayed as changing void ratio at the same depth. A pronounced change in compressibility with depth at four applied stress levels occurs between Subunits IA and IB.

undrained shear strength to the measured shear strength parameters as follows:

$$
s_{\mathrm{u}} / \sigma_{\mathrm{o}}^{\prime}=\left(s_{\mathrm{u}} / \sigma_{\mathrm{o}}^{\prime}\right)_{\mathrm{NC}} \mathrm{OCR}^{m}
$$

where $s_{\mathrm{u}}$ is undrained shear strength, $\sigma_{\mathrm{o}}^{\prime}$ is effective overburden stress, $\mathrm{NC}$ represents the normally consolidated condition, OCR is the overconsolidated ratio, and $m$ is a constant that depends on the type of sediment. On the basis of the results of CIU triaxial tests on overconsolidated samples (Fig. 27 and Table 10), a value of $m=$ 0.5 was computed. This value is lower than the typical $m$ value of 0.8 suggested by Ladd et al. (1977) based on CAU simple shear tests. The discrepancy between $m$ val- 


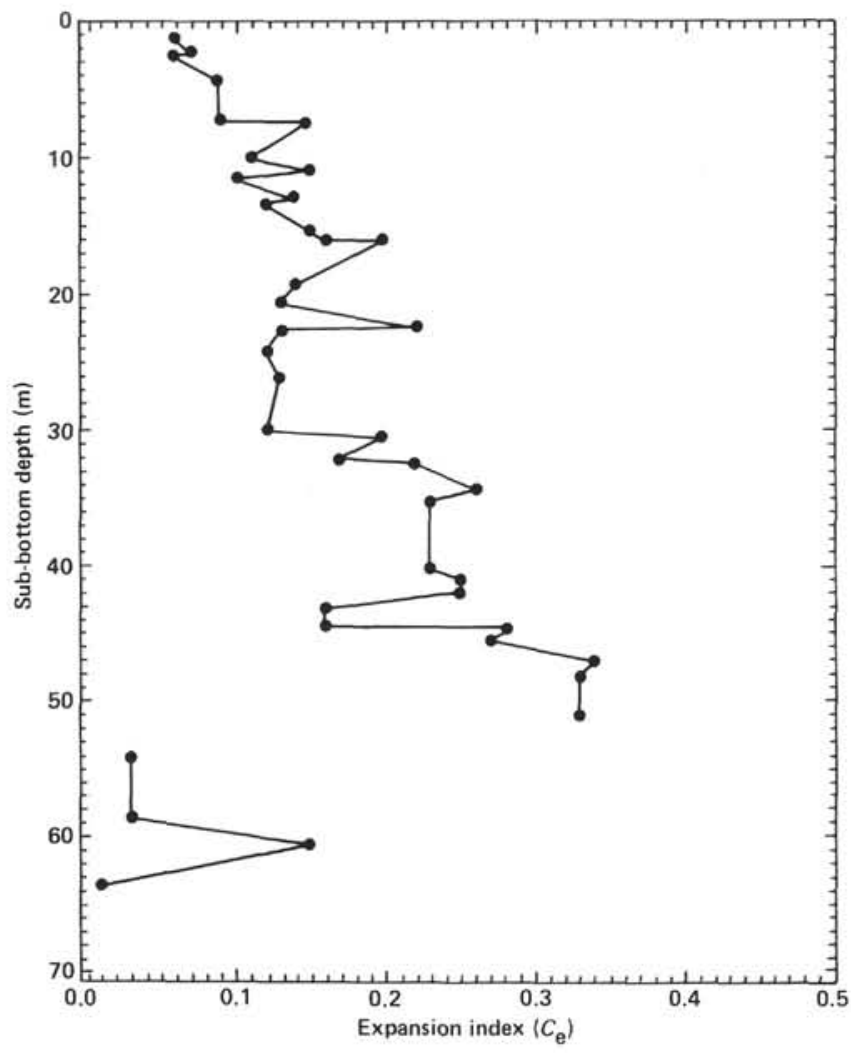

Figure 18. Expansion index-depth profile determined using the rebound portion of the $e$ versus $\log \sigma^{\prime}$ curve generated from consolidation-test results completed on Hole 576A sediments.

ues is due partly to the differences between CIU and CAU tests and partly to the differences between triaxial and simple shear tests.

In order to apply the SHANSEP method to the sediment conditions at Hole $576 \mathrm{~A}$, it is first necessary to convert the $s_{\mathrm{u}} / \sigma_{\mathrm{o}}^{\prime}$ values from the CIU tests to the in situ coefficient of earth pressure at rest condition, $K_{\mathrm{o}}$, as determined by the CAU test. A study of the data in Tables 9 and 11 indicates that the $s_{\mathrm{u}} / \sigma_{\mathrm{o}}^{\prime}$ values from the CIU tests should be multiplied by 0.89 to obtain the estimated values for the CAU condition. The adjusted values are summarized in Table 12.

The values of $s_{\mathrm{u}} / \sigma_{\mathrm{o}}^{\prime}$ reported for the upper $28 \mathrm{~m}$ of sediment in Table 12 must be further adjusted to account for the apparent in situ overconsolidated condition determined on the basis of consolidation test results. This was completed using the SHANSEP equation and a value of $m=0.5$ (Table 13) together with the OCR values determined from consolidation test results in Figure 13. The computed values of normalized in situ strength, $s_{\mathrm{u}} /$ $\sigma_{\mathrm{o}}^{\prime}$, and the in situ strength, $s_{\mathrm{u}}$, computed using SHANSEP are plotted versus depth in Figures 28 and 29, respectively.

\section{Interpretation of Shear Strength Profiles}

Interpretation of the normalized shear strength profiles presented in Figures 24 and 28 requires consideration of two factors: (1) the effects of sample disturbance and (2) the in situ state of consolidation.

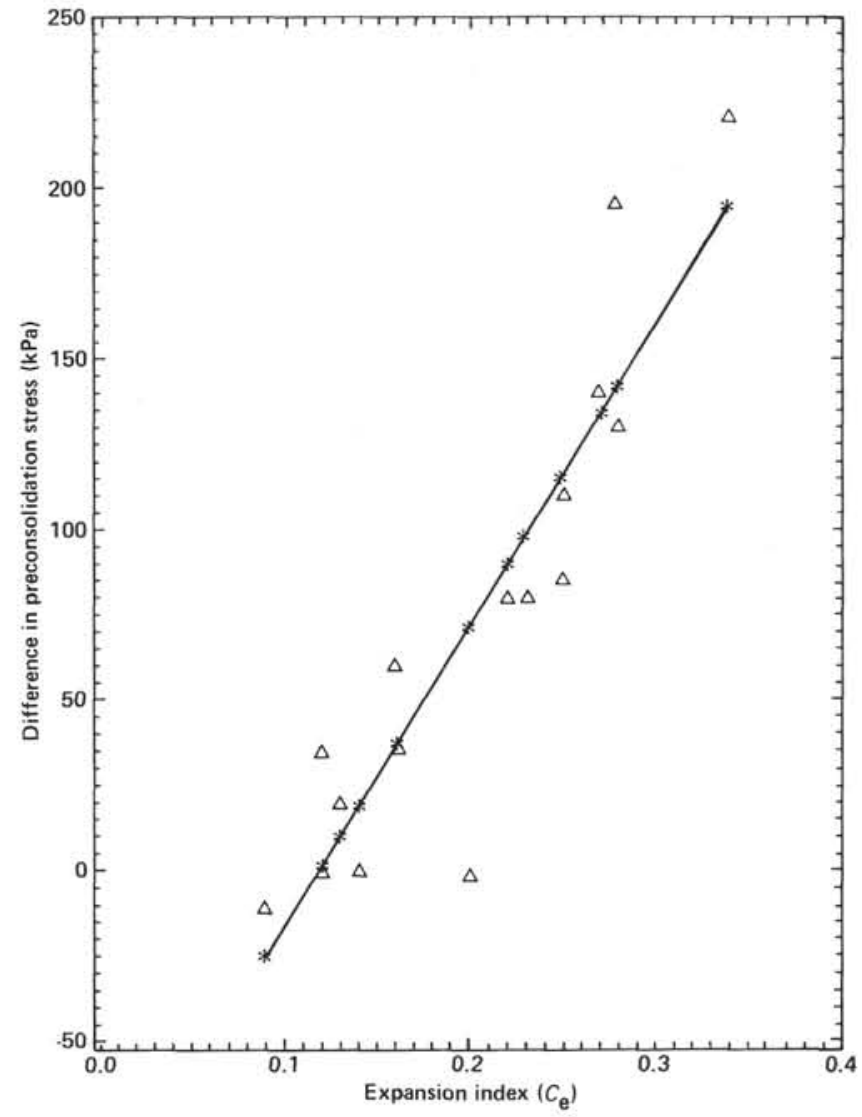

Figure 19. Relationship between the expansion index and the difference between a known preconsolidated stress determined using a hysteresis loop and the preconsolidation stress determined graphically using the Casagrande technique (see test for explanation). As the expansion index increases the difference between the known and calculated preconsolidation stress values also increase. Asterisks $\left(^{*}\right)$ represent regression analysis results $(\sigma=0.83)$.

The effects of disturbance are evident from a comparison of the normalized strength-depth profiles in Figures 24 and 28. At all depths, the undrained strengths obtained by both the vane shear and UU triaxial tests are considerably lower than those computed for the in situ condition. This decrease in shear strength is consistent with the known effects of sample disturbance on sediment shear strength.

The influence of the in situ sediment consolidation state on the undrained shear strength at Hole $576 \mathrm{~A}$ is clearly demonstrated by the data presented in Figures 24 and 28. Results of consolidation tests summarized in Figure 15 indicate that the sediments are overconsolidated to a depth of $28 \mathrm{~m}$ sub-bottom. The normalized shear strength values (Fig. 28), as well as the computed in situ values (Fig. 29) are consistent with the consolidation test results from the upper $28 \mathrm{~m}$. The shear strength results show a much higher gradient over this interval than the gradient that exists in the normally consolidated sediments between 28 and $55 \mathrm{~m}$ sub-bottom.

An examination of the computed undrained strength profile (Fig. 29) reveals the difference between the sediment strength characteristics of Subunits IA and IB. It also demonstrates the usefulness of the SHANSEP meth- 
Table 6. Summary of preconsolidation stress values calculated using Casagrande's technique and the modified techniques incorporating the sediment expansion characteristics described in the text.

\begin{tabular}{|c|c|c|c|c|c|c|}
\hline $\begin{array}{l}\text { Sub-bottom } \\
\text { depth } \\
\text { (m) }\end{array}$ & $\begin{array}{c}\sigma_{\mathrm{cl}} \\
(\mathrm{kPa})\end{array}$ & OCR & $\begin{array}{c}\sigma_{\mathrm{c} 4} \\
(\mathrm{kPa})\end{array}$ & OCR & $\begin{array}{c}\sigma_{\mathrm{c} 5} \\
(\mathrm{kPa})\end{array}$ & OCR \\
\hline 1.40 & 25 & 5.58 & 25 & 5.58 & 70 & 15.6 \\
\hline 2.45 & 76 & 10.11 & 76 & 10.11 & & \\
\hline 2.55 & 52 & 6.62 & 52 & 6.62 & & \\
\hline 3.00 & 34 & 3.60 & & & & \\
\hline 4.52 & 49 & 3.34 & 49 & 3.34 & 45 & 3.06 \\
\hline 5.00 & 57 & 3.38 & & & 54 & \\
\hline 5.60 & 88 & 4.86 & & & & \\
\hline 7.40 & 67 & 2.79 & 67 & & & \\
\hline 7.54 & 69 & 2.84 & 96 & 3.95 & 64 & 2.84 \\
\hline 9.72 & 89 & 2.89 & 89 & 2.89 & & \\
\hline 10.23 & 106 & 3.27 & 106 & 3.27 & 85 & 2.62 \\
\hline 11.11 & 121 & 3.48 & 136 & 3.91 & 110 & 3.16 \\
\hline 11.30 & & & & & 105 & \\
\hline 11.60 & 84 & 2.32 & & & 68 & 1.87 \\
\hline 11.66 & 74 & 2.03 & 74 & 2.03 & & \\
\hline 12.21 & 106 & 2.78 & & & & \\
\hline 13.15 & 152 & 3.71 & 170 & 4.15 & & \\
\hline 13.52 & 140 & 3.23 & 140 & 3.23 & 85 & 2.01 \\
\hline 15.50 & 94 & 1.93 & 121 & 2.48 & 75 & 1.53 \\
\hline 16.15 & 135 & 2.67 & 171 & 3.37 & 110 & 2.17 \\
\hline 16.22 & 135 & 2.65 & 207 & 4.07 & 97 & 1.90 \\
\hline 19.54 & 96 & 1.52 & 114 & 1.80 & 83 & 1.31 \\
\hline 20.76 & 195 & 2.87 & 208 & 3.06 & 190 & 2.79 \\
\hline 22.43 & 61 & 0.82 & 151 & 2.02 & 115 & 1.54 \\
\hline 22.65 & 73 & 0.97 & 82 & 1.08 & & \\
\hline 22.86 & 113 & 1.48 & & & & \\
\hline 24.34 & 120 & 1.45 & 120 & 1.45 & 74 & 0.89 \\
\hline 25.65 & 218 & 2.48 & & & & \\
\hline 26.02 & 96 & 1.07 & 105 & 1.17 & 85 & 0.95 \\
\hline 30.09 & 90 & 0.85 & 90 & 0.85 & 80 & 0.75 \\
\hline 30.40 & 88 & 0.82 & & & & \\
\hline 30.60 & 88 & 0.81 & 160 & 1.48 & 61 & 0.56 \\
\hline 32.15 & 145 & 1.28 & 190 & 1.67 & 125 & 1.10 \\
\hline 32.47 & 140 & 1.22 & 230 & 2.00 & & \\
\hline 34.40 & 140 & 1.15 & 281 & 2.31 & 180 & 1.48 \\
\hline 32.29 & 155 & 1.24 & 254 & 2.03 & 210 & 1.67 \\
\hline 39.37 & 113 & 0.80 & & & 110 & 0.77 \\
\hline 40.36 & 70 & 0.48 & 169 & 1.16 & 78 & 0.54 \\
\hline 41.04 & 120 & 0.81 & 237 & 1.60 & 180 & \\
\hline 41.65 & 130 & & & & & \\
\hline 42.02 & 120 & 0.78 & 237 & 1.56 & 180 & 1.18 \\
\hline 43.30 & 90 & 0.57 & 126 & 0.79 & 140 & 0.88 \\
\hline 44.16 & 109 & 0.66 & 145 & 0.88 & 180 & 1.10 \\
\hline 44.65 & 155 & 0.95 & 299 & 1.82 & 340 & 2.07 \\
\hline 44.97 & 92 & 0.56 & 236 & 1.43 & 200 & 1.21 \\
\hline 45.60 & 140 & 0.83 & 275 & 1.63 & 200 & 1.18 \\
\hline 47.20 & 75 & 0.43 & 273 & 1.56 & 185 & 1.06 \\
\hline 48.15 & 183 & 1.03 & & & & \\
\hline 48.21 & 130 & 0.73 & 319 & 1.79 & 250 & 1.40 \\
\hline 49.60 & 130 & 0.71 & & & 250 & 1.3 \\
\hline 49.65 & 67 & 0.36 & & & & \\
\hline 51.36 & 110 & 0.57 & 299 & 1.56 & 170 & 0.8 \\
\hline 53.65 & 280 & 1.39 & & & 330 & 1.6 \\
\hline 54.22 & 385 & 1.88 & & & 360 & 1.76 \\
\hline 58.72 & 400 & 1.72 & & & 150 & 0.64 \\
\hline 60.65 & 320 & 1.30 & & & 240 & 0.97 \\
\hline 63.61 & 400 & 1.50 & & & & \\
\hline
\end{tabular}

Note: The overconsolidation ratios reported are those calculated using the adjusted preconsolidation stress. Blanks indicate no data available.

od for estimation of the in situ strength from the results of laboratory tests on partially disturbed samples. This profile also represents our best estimate of the in situ undrained strength of Subunits IA and IB in Hole 576A.

The sediment cores obtained from the depths of 55 to $64 \mathrm{~m}$ sub-bottom were highly disturbuted and did not provide enough data for an adequate SHANSEP analysis of the carbonate-ooze sediments in Unit II.

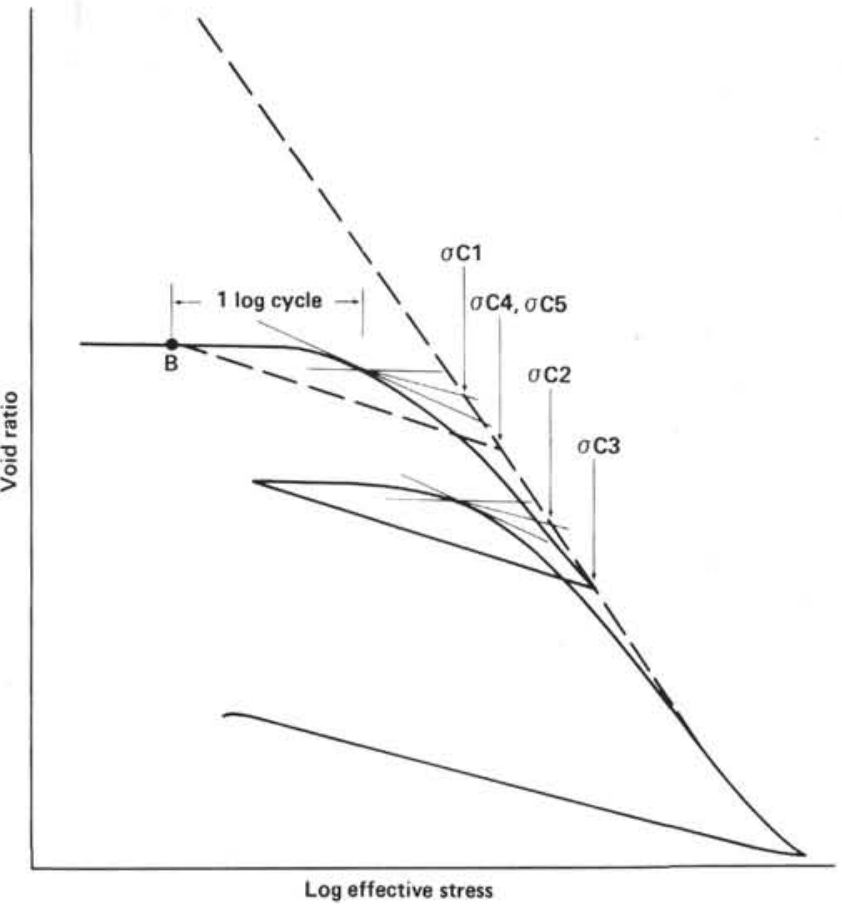

Figure 20. Hypothetical void ratio-log effective stress ( $e$ versus $\log \sigma^{\prime}$ ) curve showing how the calculated preconsolidation stress varies depending on the technique used (see explanation in text). $\sigma_{\mathrm{cl}}$ is the preconsolidation stress calculated using the Casagrande (1936) technique. $\sigma_{\mathrm{c} 2}$ is the preconsolidation stress calculated using the Casagrande technique on the hysteresis loop on the $e \log \sigma^{\prime}$ curve. $\sigma_{\mathrm{c} 3}$ is the actual preconsolidation stress for the hysteresis loop. $\sigma_{\mathrm{c} 4}$ is the preconsolidation pressure determined using the rebound characteristics (Fig. 19) and the hysteresis loop. $\sigma_{\mathrm{c} 5}$ is the preconsolidation pressure determined using point $\mathrm{B}$ and the slope of the rebound curve.

\section{DISCUSSION OF RESULTS}

The geotechnical property results for Hole 576A sediments represent the most complete geotechnical data set ever compiled for a continuously depositional sedimentary section that spans 65 m.y. Excellent agreement exists between index property, consolidation, permeability, and shear strength test data generated during the initial sampling at SIO and at the various geotechnical laboratories participating in the analyses. These results are also valuable because they demonstrate the degree of interlaboratory variation one can expect from each type of test performed provided that similar techniques are adopted.

Predicted geotechnical property results (i.e., based on the geological and geotechnical information available prior to drilling) and actual geotechnical property measurements show significant differences. Several important factors or combination of factors have influenced the geotechnical property results observed in Hole 576A sediments. These primary factors include: (1) core disturbance, (2) time, (3) cementation and interparticle bonding, (4) mineralogy, and (5) grain size.

The term core disturbance includes a number of processes. Any sampling method will result in stress relief and expansion. In addition, mechanical alteration of the sediment fabric results from the coring, handling, and 


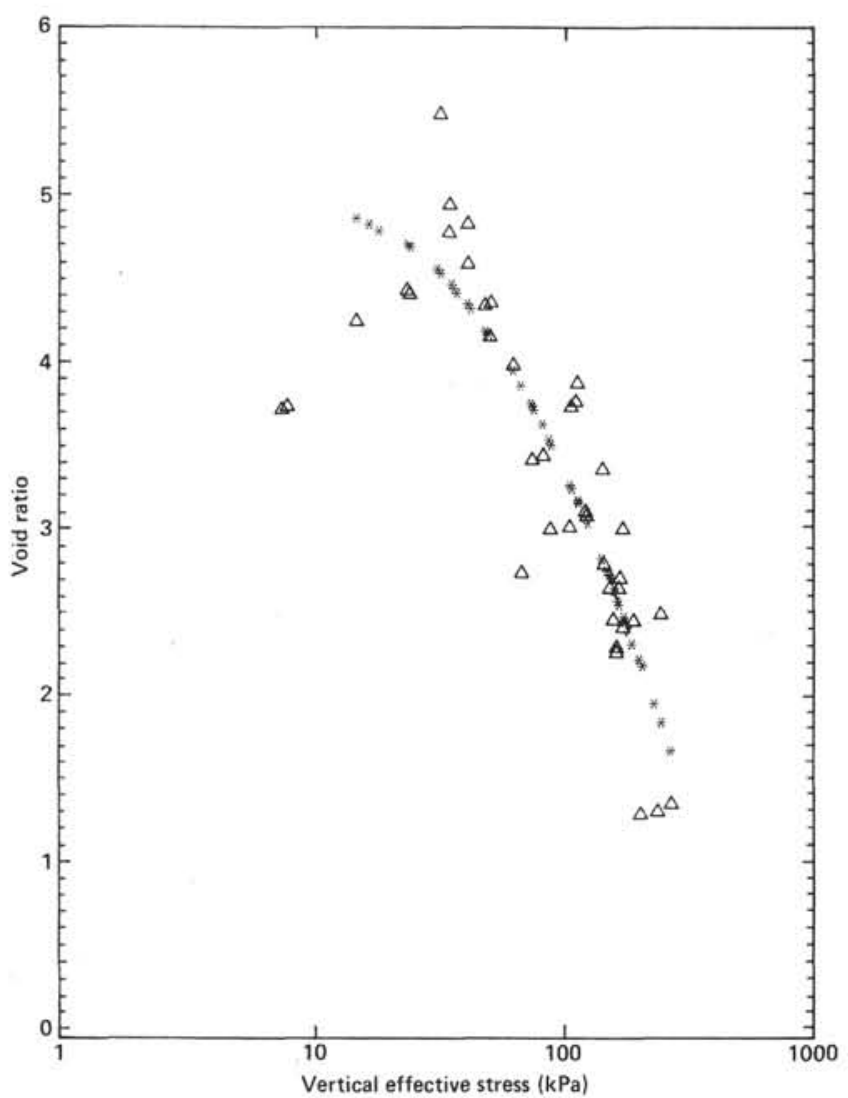

Figure 21. Void ratio-log effective stress curve determined using the void ratios calculated at the preconsolidation stress and the effective overburden stress calculated for each consolidation sample depth. The curve is representative of in situ field conditions. Asterisks $\left({ }^{*}\right)$ represent a quadratic fit to the field data $(\sigma=0.74)$.

initial testing procedures. Such effects disturb the sediment fabric and generally reduce both the shear strength and the preconsolidation stress as determined in the laboratory for a particular sample. Although difficult to quantify, a comparison of the $s_{\mathrm{u}} / \sigma_{\mathrm{o}}^{\prime}$ ratios determined using the results of laboratory tests and results corrected for in situ conditions using SHANSEP clearly demonstrates the effects of disturbance (Figs. 24 and 28).

Time has a significant effect on the geotechnical behavior of fine-grained sediments. Sedimentation rates at Site 576 were generally less than $10 \mathrm{~m} / \mathrm{m}$.y. over the last 65 m.y. These rates are sufficiently slow to allow complete dissipation of any excess pore pressures resulting from the sedimentation process. Thus, the time-dependent physical processes, (i.e., primary and secondary consolidation) have been completed or are continuing at such a slow rate that the Site 576 sediments should be very near a state of physical equilibrium.

Temporal variations in chemical processes (i.e., diagenesis, cementation, and interparticle bonding) could also influence the geotechnical behavior of fine-grained sediments. Dadey (1983) has proposed clay-particle cementation as the primary factor responsible for the overconsolidation evident in Subunit IA sediments. The cementation effect may be more pronounced in high void ratio shallow sediments where the influence of accumulated overburden relative to the effects of cementation is

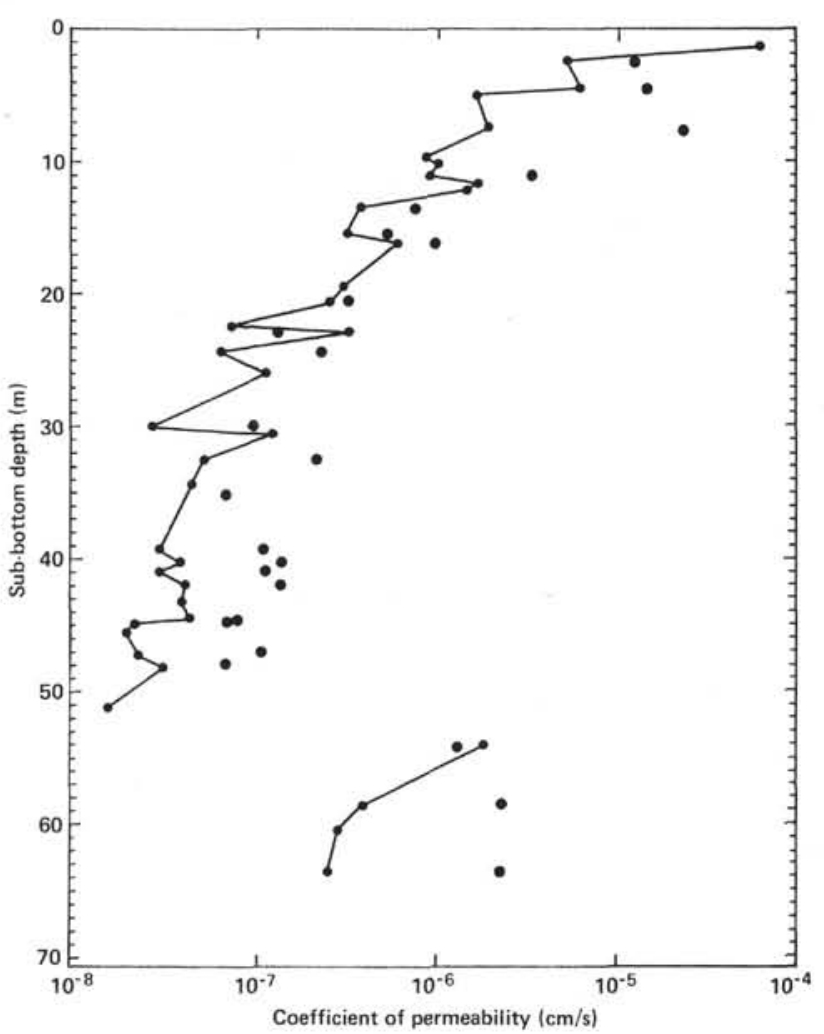

Figure 22. Coefficient of permeability-depth profile for Hole 576A sediments. Permeability values determined by direct measurement (falling head, constant head, and flow pump) are illustrated by connected dots. Permeability values calculated using consolidation theory are illustrated by solid circles $(\bullet)$. All permeability values were obtained at the preconsolidation stress.

not as significant as in more deeply buried sediments. Similar reasoning suggests that cementation effects should be reflected in the near-surface sediment shear strength. The variations observed in the shear strength gradient evident in the SHANSEP results between Subunits IA and IB may reflect these effects. However, the variability in near-surface shear strength values precludes any definitive assessment of the cementation effects on shear strength.

The most common clay minerals present in Hole 576A sediments are illite, smectite, chlorite, and kaolinite. Illite comprises approximately $50 \%$ of the clay minerals present. The quartz content ranges between approximately 3 and $17 \%$ (Table 2). The largest mineralogical variation in Hole 576A sediments is the pronounced increase in X-ray amorphous material at depth and the variable quartz content. The higher expansion indices and increased plasticity of Subunit IB sediments suggest that the geotechnical behavior of the X-ray amorphous component is similar to smectite.

Examination of the relationships between void ratio at $50 \mathrm{kPa}$ (Fig. 17), silt content (Fig. 30), and the compression index (Fig. 31) show very unusual relationships with depth, particularly in Subunit IA. As void ratio and compression index increase over this interval, silt content also increases. Examination of scanning electron micrographs obtained from the silt-size fraction revealed this component was made up of quartz grains and clay 
Table 7. Shear strength test program completed on Hole $576 \mathrm{~A}$ sediments.

\begin{tabular}{|c|c|c|c|c|c|}
\hline \multirow{2}{*}{$\begin{array}{l}\text { Core- } \\
\text { Section }\end{array}$} & \multirow{2}{*}{$\begin{array}{l}\text { Sub-bottom } \\
\text { depth } \\
\text { (m) }\end{array}$} & \multicolumn{4}{|c|}{ Test type } \\
\hline & & UU & $(\mathrm{CIU})_{\mathrm{nc}}$ & $(\mathrm{CIU})_{\mathrm{oc}}$ & $(\mathrm{CAU})_{\mathrm{nc}}$ \\
\hline $1-2$ & 2.9 & $\mathrm{x}$ & & & \\
\hline $1-3$ & 3.0 & $\mathrm{x}$ & & & \\
\hline $1-3$ & 3.9 & $\mathrm{x}$ & & & \\
\hline $1-4(2)$ & 5.5 & & $\mathrm{x}$ & & \\
\hline $1-4(1)$ & 5.6 & & $\mathrm{x}$ & & \\
\hline $1-5(1)$ & 6.3 & & $\mathrm{x}$ & & \\
\hline $1-5(2)$ & 6.4 & & $\mathrm{x}$ & & \\
\hline $2-1$ & 10.0 & $x$ & & & \\
\hline $2-4$ & 13.7 & $\mathrm{x}$ & & & \\
\hline $2-6$ & 16.8 & $\mathrm{x}$ & & & \\
\hline $2-6(1)$ & 17.3 & & $\mathrm{x}$ & & \\
\hline $2-6(2)$ & 17.4 & & $\mathrm{x}$ & & \\
\hline $2-6(3)$ & 17.5 & & $\mathrm{x}$ & & \\
\hline $3-2$ & 21.8 & $\mathrm{x}$ & & & \\
\hline $3-5$ & 21.6 & & $\mathrm{x}$ & & \\
\hline $3-4(3)$ & 24.7 & & $\mathrm{x}$ & & \\
\hline $3-4(2)$ & 24.7 & & $\mathrm{x}$ & & \\
\hline $3-4(1)$ & 24.8 & & $\mathrm{x}$ & & \\
\hline $3-6$ & 26.7 & $x$ & & & \\
\hline $4-1(1)$ & 29.2 & & & $x$ & \\
\hline $4-2(2)$ & 29.3 & & & & $\mathrm{x}$ \\
\hline $4-1(2)$ & 29.5 & & & $\mathrm{x}$ & \\
\hline $4-2(3)$ & 29.5 & & & & $x$ \\
\hline $4-1(3)$ & 29.6 & & & $x$ & \\
\hline $4-2(4)$ & 29.6 & & & & $\mathrm{x}$ \\
\hline $4-2(1)$ & 29.7 & & & & $\mathrm{x}$ \\
\hline $5-3$ & 40.2 & $\mathrm{x}$ & $\mathrm{x}$ & & \\
\hline $5-3(1)$ & 40.5 & & $\mathrm{x}$ & & \\
\hline $5-4$ & 40.7 & $\mathrm{x}$ & $\mathrm{x}$ & & \\
\hline $5-4(2)$ & 42.3 & & $\mathrm{x}$ & & \\
\hline $5-4(3)$ & 42.4 & & $\mathrm{x}$ & & \\
\hline $5-4(1)$ & 42.6 & & & & $\mathrm{x}$ \\
\hline $5-4(2)$ & 42.7 & & & & $x$ \\
\hline $5-4(3)$ & 42.9 & & & & $\mathrm{x}$ \\
\hline $5-4(4)$ & 43.0 & & & & $\mathrm{x}$ \\
\hline $5-5$ & 44.0 & $\mathrm{x}$ & & & \\
\hline $6-2$ & 48.3 & $\mathrm{x}$ & & & \\
\hline $6-2(1)$ & 48.8 & & $\mathrm{x}$ & & \\
\hline $6-2(2)$ & 49.0 & & $\mathrm{x}$ & & \\
\hline $6-3$ & 49.7 & $\mathrm{x}$ & & & \\
\hline $6-3$ & 49.8 & $\mathrm{x}$ & & & \\
\hline $6-4$ & 51.8 & $\mathrm{x}$ & & & \\
\hline $7-6$ & 64.3 & $\mathrm{x}$ & & & \\
\hline
\end{tabular}

Note: Subscripts nc and oc stand for normally consolidated and overconsolidated sediments, respectively. The test series number is shown in parentheses in the core-section column.

aggregates. No other silt-size components were identified. At $13 \mathrm{~m}$ sub-bottom quartz accounts for only $17 \%$ of the total $52 \%$ sand and silt-size fraction present. Given these relative proportions, the quartz grains are probably dispersed within the sediments and will have little or no effect on the sediments geotechnical behavior. The bonded clay aggregates comprising $35 \%$ of the coarse fraction at $13 \mathrm{~m}$ sub-bottom are sufficiently bonded to resist deflocculation. If the clays have not undergone extensive diagenetic alterations with time, then these aggregates probably result from clay particle cementation, possibly by iron oxides. In either case, these aggregates must form a high void ratio, porous structure that is dispersed among a clay particle matrix in a manner so as not to decrease the overall soil compressibility. The concept of bonded clay aggregates is not new; however, this study does present indications that clay aggregates do exist and can influence the geotechnical behavior of finegrained sediments.
In conclusion, the geotechnical property results from Hole 576A sediments, in particular the consolidation test results, appear inconsistent with the known geological history of the area. The consolidation test results are substantiated by both the anomalous relationships occurring between index properties (i.e., grain size increases as void ratio increases) and the shear-strength results. A number of possibilities have been presented to explain these observations, including core disturbance, particle cementation, formation of bonded clay aggregates, and inadequate techniques for determining the preconsolidation stress. Available information precludes definitive conclusions as to which of these possibilities is most significant, although it is likely that each process has influenced the results to some degree. These results do show conclusively, however, that conventional geotechnical relationships developed largely from terrestrial studies should not be applied to the marine environment without very careful consideration and analysis.

\section{ACKNOWLEDGMENTS}

We are indebted to the DSDP Leg 86 scientific party and operational personnel for providing the time and patience necessary to successfully complete Hole $576 \mathrm{~A}$. This study would not have been possible without the gracious assistance of Amy Altman and the DSDP curatorial staff during the core processing. Sally Frew politely persevered while typing and retyping this report. Phil Valent, Mike Riggins, and Bob Prindle kindly agreed to review this manuscript and provided innumerable helpful comments. Partial financial support was provided by the Department of Energy through contracts with Sandia National Laboratories (DOE-AC04-76DP00789) and Argonne National Laboratory (DOE-31-109-ENG-38).

\section{REFERENCES}

American Society for Testing and Materials, 1984. Annual Book of ASTM Standards, Part 19, Natural Building Stones; Soil and Rock: Philadelphia (ASTM).

Bishop, A. W., Webb, D. L., and Lewin, P. I., 1965. Undisturbed samples of London clay from the Ashford common shaft: Strengtheffective stress relationship. Geotechnique, 15:1-31.

Bishop, A. W., and Henkel, D. J., 1962. The Measurement of Soil Properties in The Triaxial Test: London (Edward Arnold, Ltd.).

Brumund, W. F., and Callender, G. W., Jr., 1975. Compressibility of undisturbed submarine sediments. Proc. Civ. Eng. Oceans/III, Am. Soc. Civ. Eng., 1:363-379.

Brumund, W. F., Jonas, E., and Ladd, C. C., 1976. Estimating in situ maximum past (preconsolidation) pressure of saturated clays from results of laboratory consolidometer tests. Transp. Res. Board, Spec. Rept. 163:4-12.

Burmister, B. M., 1951. The application of controlled test methods in consolidation testing. Consolidation Testing of Soils, ASTM Spec. Tech. Publ. 126:83-91.

Casagrande, A., 1936. The determination of the preconsolidation load and its practical significance. Proc. Int. Conf. Soil Mech. Found. Eng., 1st, 3:60-64. 1948. Classification and identification of soils. Trans. Am Soc. Civ. Eng., 113:901.

Cooling, L. F., and Skempton, A. W., 1942. A laboratory study of London clay. J. Inst. Civ. Eng., 17:251-256.

Dadey, K. A., 1983. Stress history of unlithified marine sediments in the northwest Pacific [M.S. thesis]. University of Rhode Island, Kingston.

Damuth, J. E., Jacobi, R. D., and Hayes, D. E., 1983. Sedimentation processes in the Northwest Pacific basin revealed by echo-character mapping studies. Geol. Soc. Am. Bull., 94:381-395.

Folk, R. L., 1974. Petrology of Sedimentary Rocks: Austin (Hemphill Publ. Co.).

Gorman, C. T., Hopkins, T. C., Deep, R. C., and Drnevich, V. P., 1978. Constant-rate of strain and controlled gradient consolidation testing. Geotech. Test. J., 1:3-15. 
Griffin, J. J., Windom, H., and Goldberg, E. D., 1968. The distribution of clay minerals in the world ocean. Deep Sea Res., 15: 433-459.

Hamilton, E. L., 1964. Consolidation characteristics and related properties of sediments from experimental mohole (Guadalupe site). J. Geophys. Res., 69:4257-4269.

1965. Sound speed and related physical properties of sediments of experimental mohole (Guadalupe site). Geophysics, 30: 257-261.

Heath, G. R., and Pisias, N. G., 1979. A method for the quantitative estimation of clay minerals in North Pacific deep-sea sediments. Clays Clay Miner., 27:175-184.

Ladd, C. C., and Foott, R., 1976. New design procedure for stability of soft clays. J. Geotech. Eng. Div. Am. Soc. Civ. Eng., pp. 763-786.

Ladd, C. C., Foott, R., Ishikara, K., Schlosser, S., and Paulos, H. G., 1977. Stress-deformation and strength characteristics, state of art report. Int. Conf. Soil Mech. Found. Eng., 9th, ISSMFE, Tokyo.

Lambe, T. W., 1951. Soil Testing for Engineers. New York (Wiley).

Lee, H. J., 1984. State of the art: Laboratory determination of the strength of marine soils. Presented at ATSM Symp. Lab. In Situ Deter. Strength Mar. Soils, San Diego.

Leinen, M., and King, T. W., 1985. Mineralogy of North Pacific sediments from North Pacific candidate sites. In Shephard, L. E. (Ed.), 1983 Subseabed Disposal Program Annual Report: Site Assessment, Sandia National Laboratories, pp. 113-140.

Lowe, J., III., Zaccheo, P. F., and Feldman, H. S., 1964. Consolidation with back pressure. J. Soil Mech. Found. Div., Am. Soc. Civ. Eng., 90:69-86.

Noorany, I., 1984. Phase relations in marine soils. J. Geotech. Eng. Div., Am. Soc. Civ. Eng., 110: 539-543.

Olsen, H. W., 1966. Darcy's Law in saturated kaolinite. Water Resour. Res., 2:287-295.

Pane, V., Croce, P., Znidarcic, D., Ko, H.-Y., Olsen, H. W., and Schiffman, R. L., 1983. Effects of consolidation on permeability measurements for soft clay. Geotechnique, 33:67-72.
Sangrey, D., 1977. Marine geotechnology: State of the art. Mar. Geotechnol., 2:45-80.

Scully, R. W., Schiffman, R. L., Olson, H. W., and Ko, H.-Y., 1984. Validation of consolidation properties of phosphatic clay at very high void ratios. Am. Soc. Civ. Eng. Symp. Consol. Settling, pp. 158-181.

Schmertmann, J. H., 1955. The undisturbed consolidation behavior of clay. Trans. Am. Soc. Civ. Eng., 120:1201-1227.

Shephard, L. E., Bryant, W. R., and Dunlap, W. A., 1978. Consolidation characteristics and excess pore water pressures of Mississippi Delta sediments. Proc. Annu. Offshore Technol. Conf., Pap. 3167:1037-1048.

Silva, A. J., Hetherman, J. R., and Calnan, D. I., 1981. Low gradient permeability testing of fine-grained marine sediments. ASTM Spec. Tech. Publ., 746:121-136.

Silva, A. J., Jordon, S. A., and Levy, W. P., 1983. Geotechnical Studies for Subseabed Disposal High Level Radioactive Wastes, Progress Report No. 9, Contract No. 37-2235. Albuquerque (Sandia National Laboratories).

Silva, A. J., Laine, E. P., Lipkin, J., Heath, G. R., and Akers, S. A., 1980. Geotechnical properties of sediments from North Pacific and Northern Bermuda Rise. Proc. Mar. Technol. Soc., pp. 491-499.

Skempton, A. W., 1970. The consolidation of clays by gravitational compaction. Q. J. Geol. Soc. London, 125:373-411.

Terzaghi, K., 1956. Varieties of submarine slope failures. Proc. Conf. Soil Mech. Found. Eng., 8th Texas, pp. 1-41.

Znidarcic, D., 1982. Laboratory determination of consolidation properties of cohesive soil [Ph.D. Dissert.]. University of Colorado, Boulder, Colorado.

Znidarcic, D., and Schiffman, R. L., 1983. Constant rate of deformation consolidation testing. Boulder (Department of Civil Engineering, University of Colorado).

Date of Initial Receipt: 23 May 1984

Date of Acceptance: 25 September 1984 


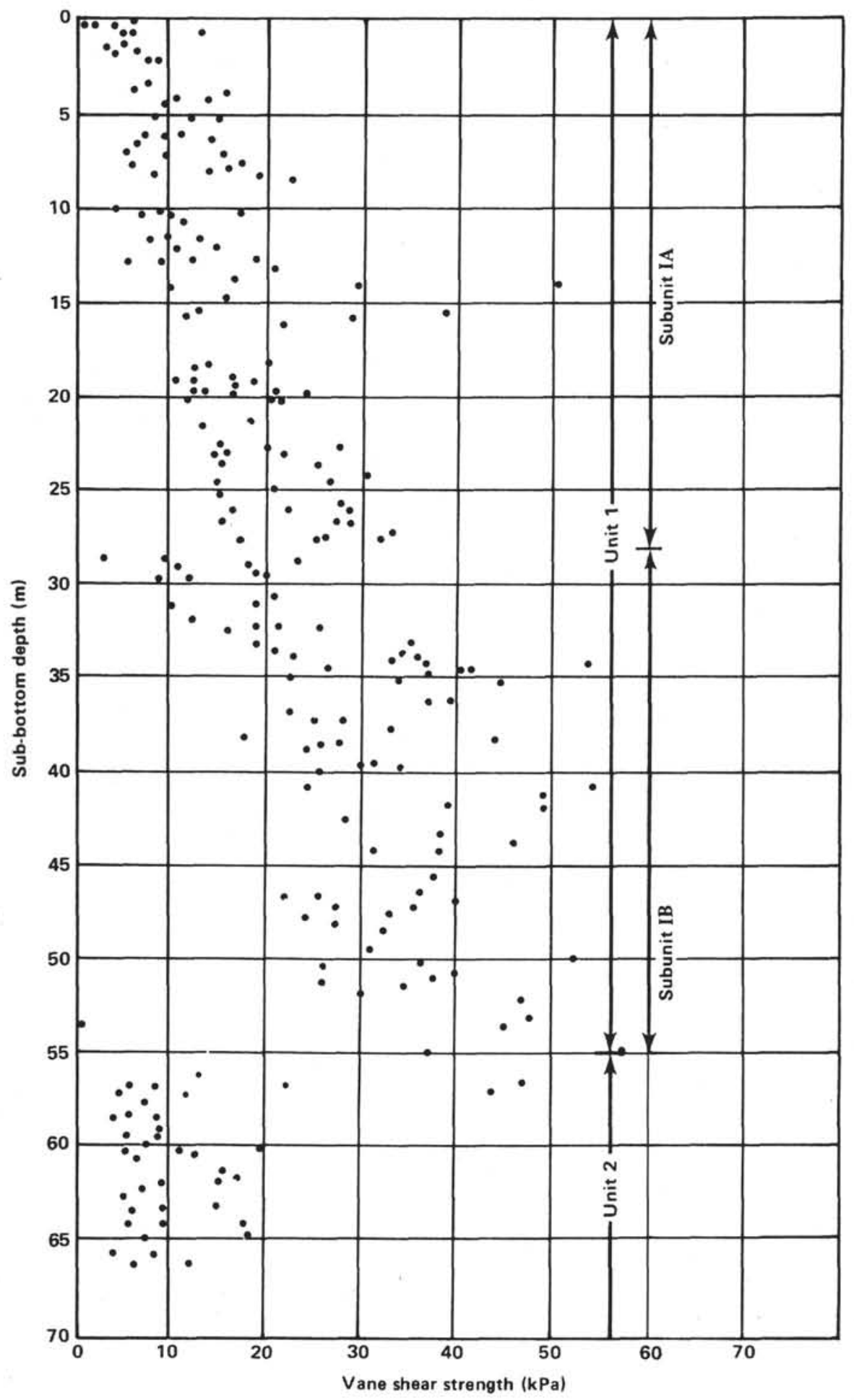

Figure 23. Laboratory vane shear strength-depth profile for Hole 576A sediments. 
Table 8. Summary of unconsolidated-undrained (UU) triaxial test results for Hole 576A sediments.

\begin{tabular}{cccccc}
\hline $\begin{array}{c}\text { Core- } \\
\text { Section }\end{array}$ & $\begin{array}{c}\text { Sub-bottom } \\
\text { depth } \\
(\mathrm{m})\end{array}$ & $\begin{array}{c}\sigma_{\mathrm{O}}{ }^{\prime} \\
(\mathrm{kPa})\end{array}$ & $\begin{array}{c}\text { Water } \\
\text { content } \\
(\%)\end{array}$ & $\begin{array}{c}\text { Triaxial } \\
\text { shear strength } \\
(\mathrm{kPa})\end{array}$ & $s_{\mathrm{u}} / \sigma_{\mathrm{o}}{ }^{\prime}$ \\
\hline $1-2$ & 2.9 & 10.9 & 153 & 14.7 & 1.34 \\
$1-3$ & 3.0 & 11.3 & 155 & 13.7 & 1.21 \\
$1-3$ & 3.9 & 14.7 & 154 & 14.3 & 0.97 \\
$2-1$ & 10.0 & 37.7 & 191 & 12.8 & 9.34 \\
$2-4$ & 13.7 & 51.7 & 160 & 11.4 & 0.22 \\
$2-6$ & 16.8 & 63.4 & 182 & 15.6 & 0.25 \\
$3-2$ & 21.8 & 82.3 & 121 & 17.6 & 0.21 \\
$3-5$ & 24.5 & 92.5 & 140 & 18.9 & 0.20 \\
$3-6$ & 26.7 & 100.8 & 114 & 29.6 & 0.29 \\
$4-2$ & 30.4 & 114.7 & 147 & 16.7 & 0.15 \\
$4-3$ & 31.0 & 116.9 & 158 & 18.3 & 0.16 \\
$4-5$ & 34.6 & 130.6 & 123 & 26.6 & 0.20 \\
$4-5$ & 34.7 & 130.9 & 130 & 42.1 & 0.32 \\
$5-3$ & 40.2 & 151.7 & 145 & 26.6 & 0.18 \\
$5-4$ & 40.7 & 153.6 & 105 & 39.3 & 0.26 \\
$5-5$ & 44.0 & 166 & 109 & 32.8 & 0.20 \\
$6-2$ & 48.3 & 182 & 101 & 33.0 & 0.18 \\
$6-3$ & 49.7 & 187.5 & 126.4 & 24.2 & 0.13 \\
$6-3$ & 49.8 & 188 & 114 & 30.0 & 0.16 \\
$6-4$ & 51.8 & 195 & 99 & 29.0 & 0.15 \\
$7-6$ & 64.3 & 243 & 117.7 & 38.0 & 0.16 \\
\hline & & & & &
\end{tabular}

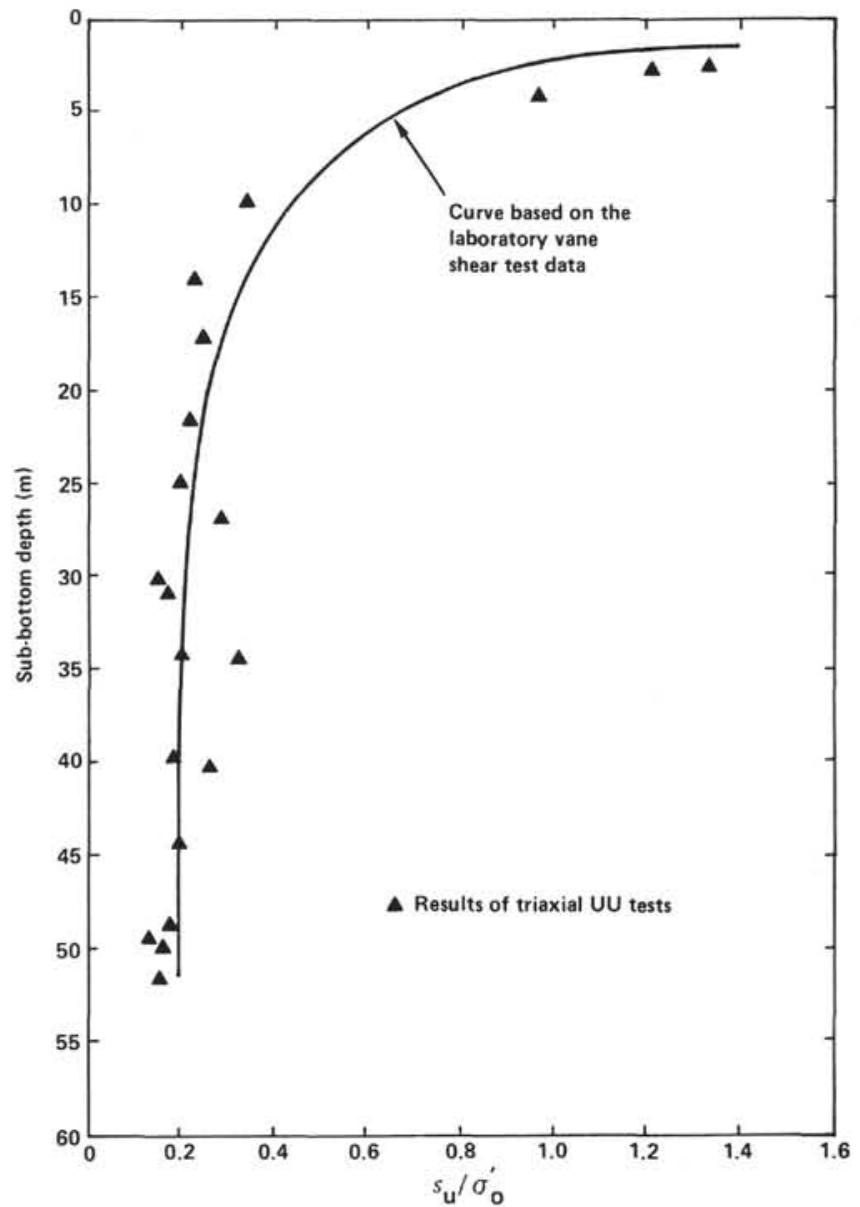

Figure 24. Normalized shear strength data comparing vane shear results with UU test results for Hole 576A sediments. 

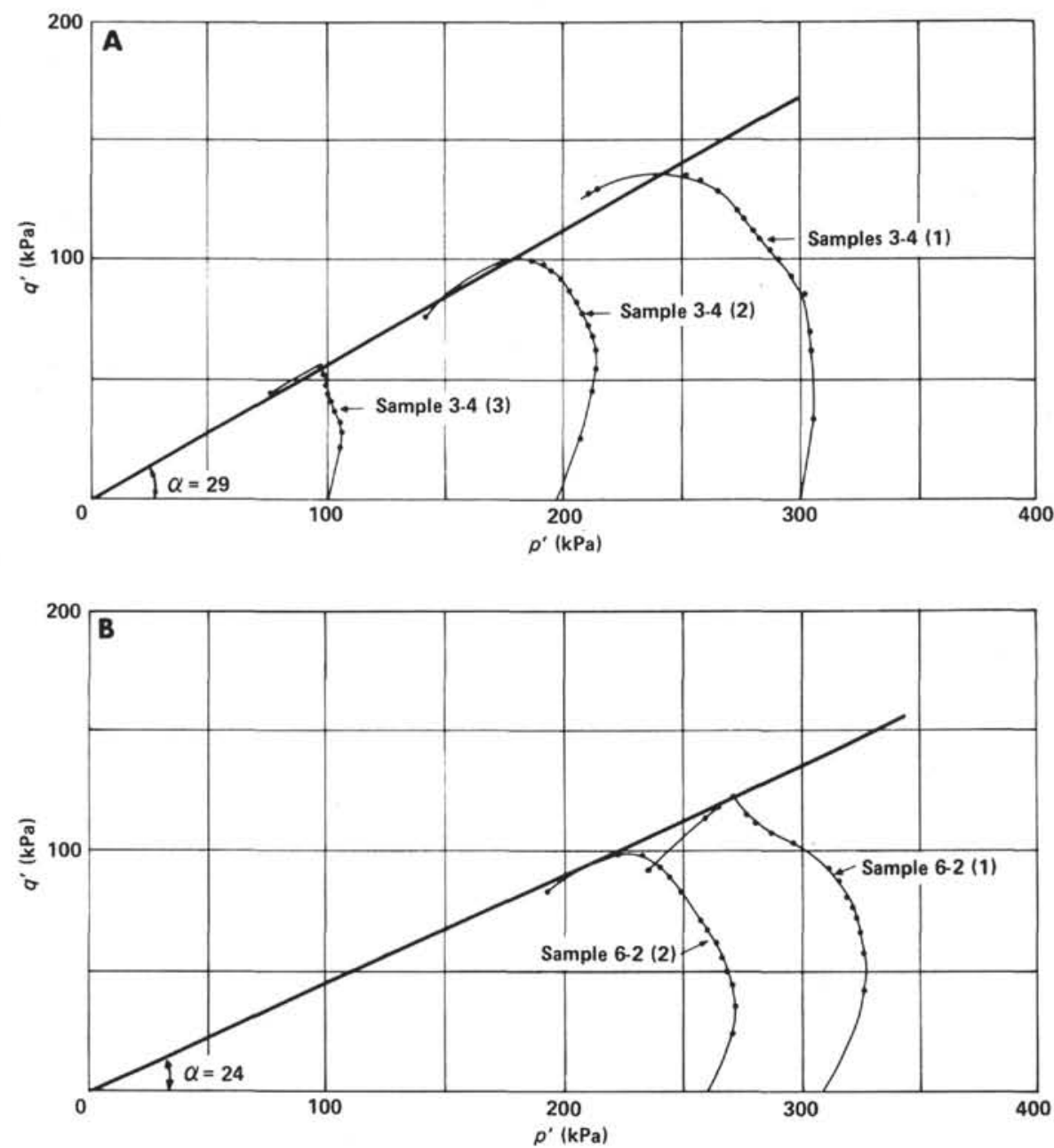

Figure 25. Example of isotropic consolidated undrained (CIU) triaxial test results from Hole 576A sediments located in Subunit IA (A, $24.70 \mathrm{~m}$ sub-bottom, $c=0, \phi=34^{\circ}$ ) and Subunit IB (B, 48.90 m sub-bottom, $\left.c^{\prime}=0, \phi=26^{\circ}\right)$. Other CIU test results are summarized in Table 9.

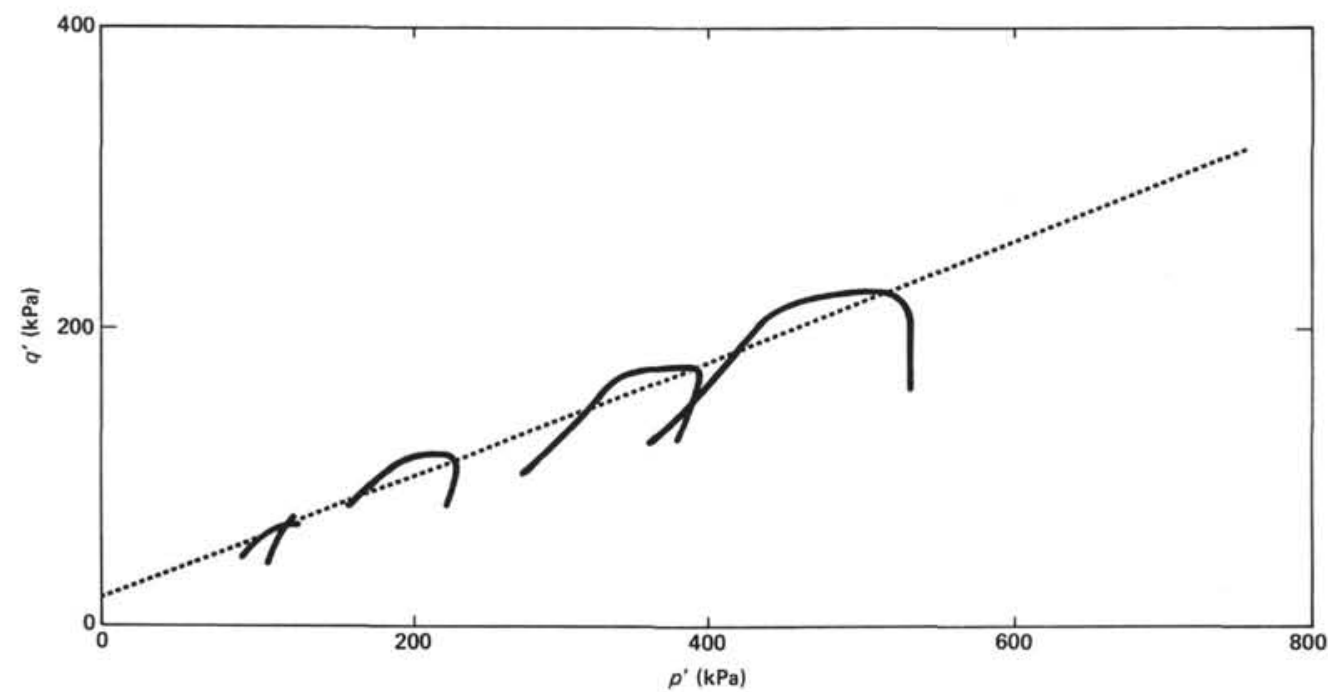

Figure 26. Results of consolidated, anisotropic undrained (CAU) $K_{\mathrm{o}}$ triaxial test results from sediment located at $30.50 \mathrm{~m}$ sub-bottom $\left(C^{\prime}=17.6 \mathrm{kPa}, \phi=23^{\circ}\right)$. Other CAU- $K_{\mathrm{o}}$ test results are summarized in Table 8. 
Table 9. Summary of CIU triaxial test results for Hole 576A sediments.

\begin{tabular}{|c|c|c|c|c|c|c|c|c|c|c|c|c|}
\hline $\begin{array}{l}\text { Test } \\
\text { no. }\end{array}$ & Core-Section & $\begin{array}{l}\text { Sub-bottom } \\
\text { depth } \\
\text { (m) }\end{array}$ & $\begin{array}{l}w_{0} \\
(\%)\end{array}$ & $\begin{array}{l}w_{\mathrm{f}} \\
(\%)\end{array}$ & $\begin{array}{c}\sigma_{\mathrm{O}^{\prime}} \\
(\mathrm{kPa})\end{array}$ & $\begin{array}{c}\sigma_{3} \\
(\mathrm{kPa})\end{array}$ & $\begin{array}{c}u_{\mathrm{i}} \\
(\mathrm{kPa})\end{array}$ & $\begin{array}{c}\sigma_{\mathrm{c}^{\prime}}^{\prime} \\
(\mathrm{kPa})\end{array}$ & B & OCR & $\begin{array}{c}s_{\mathrm{U}} \\
(\mathrm{kPa})\end{array}$ & $s_{\mathrm{u}} / \sigma_{\mathrm{O}}{ }^{\prime}$ \\
\hline 1 & $1-4(1)$ & 5.6 & 192 & 138 & 20 & 392 & 294 & 98 & 0.98 & 1 & 60.8 & 0.62 \\
\hline 2 & $1-4(2)$ & 5.5 & 199 & 131 & 20 & 441 & 294 & 147 & 0.98 & 1 & 70.6 & 0.48 \\
\hline 3 & $1-5(1)$ & 6.3 & 216 & 164 & 22 & 392 & 294 & 98 & 0.99 & 1 & 59.8 & 0.61 \\
\hline 4 & $1-5(2)$ & 6.4 & 189 & 164 & 22 & 353 & 294 & 59 & 0.99 & 1 & 45.1 & 0.76 \\
\hline 5 & $2-6(1)$ & 17.3 & 154 & 122 & 57 & 540 & 393 & 147 & 0.98 & 1 & 73.5 & 0.50 \\
\hline 6 & $2-6(2)$ & 17.4 & 153 & 137 & 57 & 491 & 393 & 98 & 0.98 & 1 & 48.0 & 0.49 \\
\hline 7 & $2-6(3)$ & 17.5 & 155 & 105 & 57 & 601 & 393 & 208 & 0.99 & 1 & 84.0 & 0.40 \\
\hline 8 & $3-4(1)$ & 24.8 & 113 & 76 & 85 & 588 & 294 & 94 & 0.99 & 1 & 132.3 & 0.45 \\
\hline 9 & $3-4(2)$ & 24.7 & 115 & 89 & 85 & 490 & 294 & 196 & 0.99 & 1 & 98.0 & 0.50 \\
\hline 10 & $3-4(3)$ & 24.6 & 127 & 121 & 85 & 392 & 294 & 98 & 0.98 & 1 & 53.9 & 0.55 \\
\hline 11 & $5-3(1)$ & 40.5 & 134 & 130 & 145 & 621 & 442 & 179 & 0.99 & 1 & 62.5 & 0.35 \\
\hline 12 & $5-4(2)$ & 42.3 & 103 & 78 & 155 & 690 & 306 & 384 & 0.99 & 1 & 145.0 & 0.38 \\
\hline 13 & $5-4(3)$ & 42.4 & 103 & 91 & 155 & 414 & 228 & 186 & 0.98 & 1 & 83.0 & 0.45 \\
\hline 14 & $6-2(1)$ & 48.8 & 124 & 102 & 180 & 598 & 294 & 304 & 0.98 & 1 & 117.6 & 0.39 \\
\hline 15 & $6-2(2)$ & 49.0 & 125 & 109 & 180 & 549 & 294 & 255 & 0.99 & 1 & 95.1 & 0.37 \\
\hline
\end{tabular}

Note: $w_{\mathrm{O}}=$ initial water content (assuming $35 \mathrm{ppm}$ dissolved salt in pore fluid); $w_{\mathrm{f}}=$ water content at failure; $\sigma_{\mathrm{O}}{ }^{\prime}=$ in situ effective stress; $\sigma_{3}=$ cell pressure; $u_{\mathrm{i}}=$ back pressure; $\sigma_{\mathrm{c}}{ }^{\prime}=\sigma_{3}-u_{\mathrm{i}}=$ consolidation pressure; $\mathrm{B}=$ pore-pressure coefficient; $\mathrm{OCR}=$ overconsolidation ratio; $s_{\mathrm{u}}=1 / 2\left(\sigma_{1}-\sigma_{3}\right)_{\max }=$ shear strength.

Table 10. Summary of CIU triaxial test results on overconsolidated samples from Hole 567A sediments.

\begin{tabular}{lccccccccccc}
\hline Sample & $\begin{array}{c}\text { Sub-bottom } \\
\text { depth } \\
(\mathrm{m})\end{array}$ & $\begin{array}{c}w_{\mathrm{O}} \\
(\%)\end{array}$ & $\begin{array}{c}w_{\mathrm{f}} \\
(\%)\end{array}$ & $\begin{array}{c}\sigma_{\mathrm{O}^{\prime}} \\
(\mathrm{kPa})\end{array}$ & $\begin{array}{c}\sigma_{3} \\
(\mathrm{kPa})\end{array}$ & $\begin{array}{c}u_{\mathrm{i}} \\
(\mathrm{kPa})\end{array}$ & $\begin{array}{r}\sigma_{\mathrm{c}^{\prime}} \\
(\mathrm{kPa})\end{array}$ & $\mathrm{B}$ & OCR & $\begin{array}{c}s_{\mathrm{U}} \\
(\mathrm{kPa})\end{array}$ & $s_{\mathrm{u}^{\prime}} / \sigma_{\mathrm{o}}{ }^{\prime}$ \\
\hline $4-1(1)$ & 29.2 & 145 & 101 & 100 & 442 & 393 & 49 & 0.97 & 8 & 94.1 & 1.92 \\
$4-1(2)$ & 29.5 & 148 & 101 & 105 & 559 & 393 & 98 & 0.96 & 4 & 108.8 & 1.11 \\
$4-1(3)$ & 29.6 & 144 & 96 & 105 & 589 & 393 & 196 & 0.98 & 2 & 134.3 & 0.69 \\
\hline
\end{tabular}

Note: See Table 9 for explanation of symbols used in column headings.

Table 11. Summary of CAU triaxial test results for Hole 576A sediments.

\begin{tabular}{|c|c|c|c|c|c|c|c|c|c|c|c|c|c|}
\hline $\begin{array}{l}\text { Test } \\
\text { no. }\end{array}$ & Sample & $\begin{array}{l}\text { Sub-bottom } \\
\text { depth } \\
\text { (m) }\end{array}$ & $\begin{array}{c}\sigma_{1}^{\prime} \\
(\mathrm{kPa})\end{array}$ & $\begin{array}{c}\sigma_{3} f^{\prime} \\
(\mathrm{kPa})\end{array}$ & $\begin{array}{l}\text { Multiple } \\
\text { of over- } \\
\text { burden }\end{array}$ & $\begin{array}{l}w_{\mathrm{o}} \\
(\%)\end{array}$ & $\begin{array}{c}w_{\mathrm{f}} \\
(\%)\end{array}$ & $\begin{array}{c}\sigma_{\mathrm{O}}^{\prime} \\
(\mathrm{kPa})\end{array}$ & $\begin{array}{c}q_{\mathrm{f}} \\
(\mathrm{kPa})\end{array}$ & $\underset{(\mathrm{kPa})}{p_{\mathrm{f}}^{\prime}}$ & $\begin{array}{c}u_{\mathrm{f}} \\
(\mathrm{kPa})\end{array}$ & $A_{\mathrm{f}}$ & $s_{\mathrm{u}} / \sigma_{\mathrm{o}}^{\prime}$ \\
\hline \multicolumn{14}{|c|}{$\phi^{\prime}=23^{\circ} C^{\prime}=17.6 \mathrm{kPa}$} \\
\hline 1 & $4-2(1)$ & 29.8 & 143 & 67 & 1.5 & 146 & 118 & 149 & 65 & 121 & 10.4 & 0.08 & 0.44 \\
\hline 2 & $4-2(2)$ & 29.4 & 300 & 144 & 3.2 & 144 & 103 & 305 & 113 & 232 & 26.7 & 0.12 & 0.37 \\
\hline 3 & $4-2(3)$ & 29.5 & 498 & 249 & 5.3 & 144 & 93 & 495 & 173 & 384 & 41.5 & 0.12 & 0.35 \\
\hline 4 & 4-2(4) & 29.6 & 694 & 376 & 7.3 & 138 & 82 & 682 & 223 & 521 & 81.3 & 0.18 & 0.33 \\
\hline \multicolumn{14}{|c|}{$\phi^{\prime}=21^{\circ} C^{\prime}=29.8 \mathrm{kPa}$} \\
\hline 5 & $5-4(1)$ & 42.6 & 200 & 93 & 1.5 & 112 & 98.5 & 202 & 82 & 150 & 27.0 & 0.16 & 0.41 \\
\hline 6 & $5-4(2)$ & 42.8 & 338 & 170 & 2.5 & 111 & 89.9 & 331 & 120 & 244 & 45.9 & 0.19 & 0.36 \\
\hline 7 & $5-4(3)$ & 42.9 & 475 & 276 & 4 & 110 & 81.6 & 475 & 164 & 356 & 80.7 & 0.25 & 0.35 \\
\hline 8 & $5-4(4)$ & 43.0 & 766 & 490 & 8 & 111 & & 766 & 237 & 582 & 141.0 & 0.30 & 0.31 \\
\hline
\end{tabular}

Note: See Table 9 for explanation of symbols used in column headings. $q_{\mathrm{f}}=$ deviation stress at failure; $p_{\mathrm{f}}{ }^{\prime}=$ mean effective stress at failure; $u_{\mathrm{f}}=$ pore pressure at failure; $A_{\mathrm{f}}=$ pore-pressure coefficient at failure. 


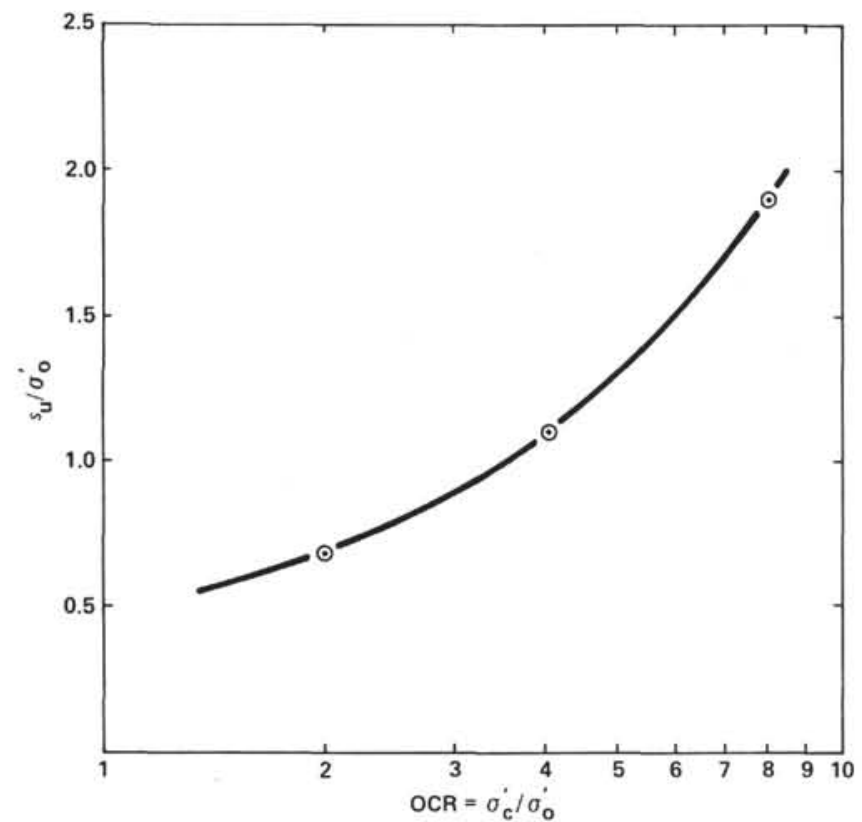

Figure 27. Relationship of the normalized shear strength with varying overconsolidation ratios developed using the results of CIU tests on sediments at $28.70 \mathrm{~m}$ sub-bottom.

Table 12. Summary of $s_{\mathrm{u}} / \sigma_{\mathrm{O}}^{\prime}$ values determined from $\mathrm{CIU}$ triaxial tests and adjusted for the in situ $\mathrm{K}_{\mathrm{O}}$ condition.

\begin{tabular}{|c|c|c|c|c|}
\hline $\begin{array}{l}\text { Core- } \\
\text { Section }\end{array}$ & $\begin{array}{l}\text { Sub-bottom } \\
\text { depth } \\
\text { (m) }\end{array}$ & OCR & $\begin{array}{l}\text { CIU tests } \\
s_{\mathrm{u}^{\prime} / \sigma_{\mathrm{o}}}{ }^{\prime}\end{array}$ & $\begin{array}{c}s_{\mathrm{u}^{\prime}} / \sigma_{\mathrm{O}}^{\prime} \\
K_{\mathrm{o}} \text { condition }\end{array}$ \\
\hline
\end{tabular}

Data from CIU test results

$\begin{array}{rrrrr}1-4 & 5.5 & 1 & 0.55 & 0.49 \\ 1-5 & 6.3 & 1 & 0.61 & 0.54 \\ 2-6 & 17.4 & 1 & 0.46 & 0.41 \\ 3-4 & 24.7 & 1 & 0.50 & 0.44 \\ 5-3 & 42.3 & 1 & 0.39 & 0.35 \\ 6-2 & 48.9 & 1 & 0.38 & 0.34\end{array}$

Data from CAU test results

\begin{tabular}{llll}
$4-2$ & 29.6 & 1 & 0.37 \\
$5-4$ & 42.8 & 1 & 0.36 \\
\hline
\end{tabular}

Table 13. Summary of $s_{\mathrm{u}} / \sigma_{\mathrm{o}}^{\prime}$ values for both the normally consolidated condition and after correcting for the state of overconsolidation.

\begin{tabular}{ccccc}
\hline $\begin{array}{c}\text { Sub-bottom } \\
\text { depth } \\
(\mathrm{m})\end{array}$ & $\left(s_{\mathrm{u}} / \sigma_{\mathrm{o}}{ }^{\prime}\right) \mathrm{NC}$ & OCR & $\left(s_{\mathrm{u}} / \sigma_{\mathrm{O}}{ }^{\prime}\right)$ & $\begin{array}{c}s_{\mathrm{u}} \\
(\mathrm{kPa})\end{array}$ \\
\hline 5.5 & 4.9 & 5.0 & 1.1 & 22 \\
6.3 & 0.54 & 4.0 & 1.1 & 24 \\
17.4 & 0.41 & 2.5 & 0.65 & 37 \\
24.7 & 0.44 & 1.5 & 0.53 & 45 \\
29.6 & 0.37 & 1.0 & 0.46 & 48 \\
42.3 & 0.35 & 1.0 & 0.35 & 54 \\
42.8 & 0.36 & 1.0 & 0.36 & 56 \\
48.9 & 0.34 & 1.0 & 0.34 & 61 \\
\hline
\end{tabular}

Note: For comparison with data in Figure 23, the shear strength values $\left(s_{\mathrm{u}}\right)$ corrected for in situ conditions using SHANSEP are also shown.

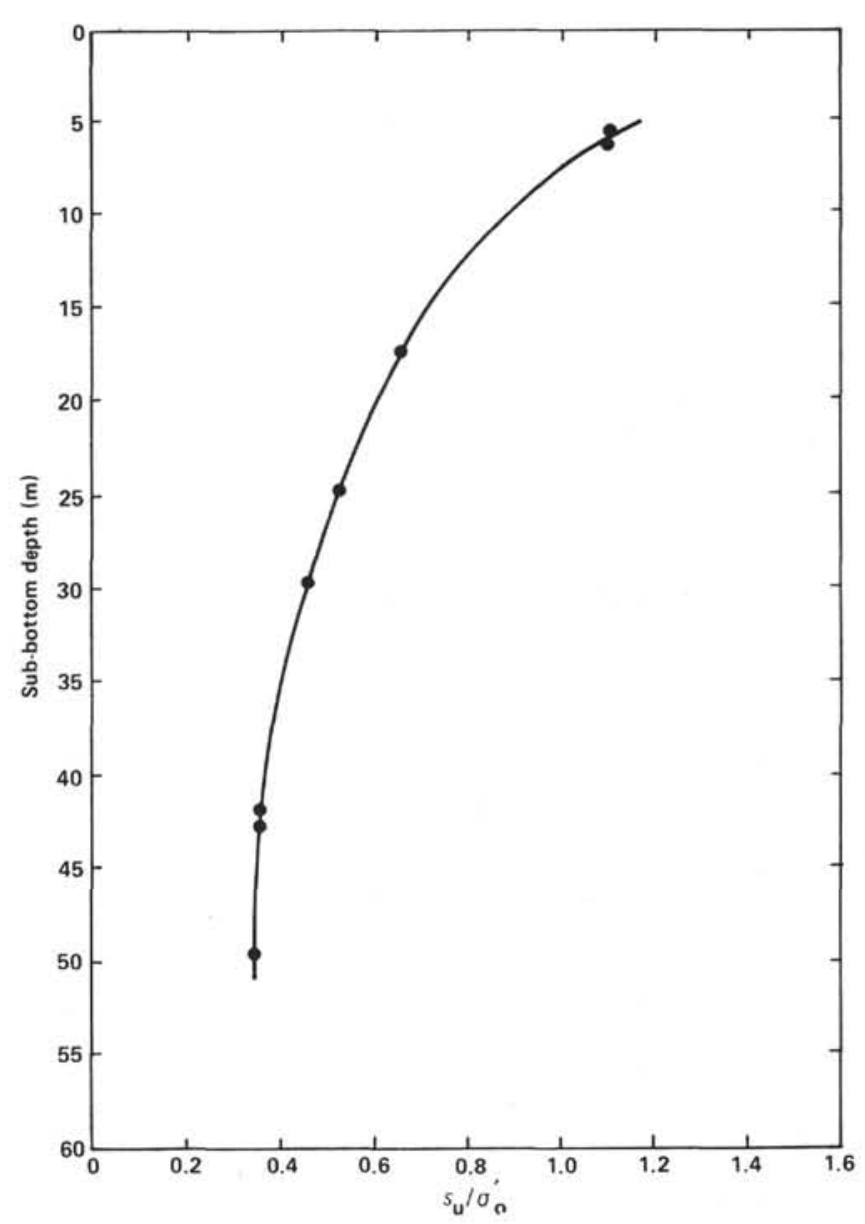

Figure 28. Variations in the ratio of $s_{\mathrm{u}} / \sigma_{\mathrm{o}}^{\prime}$ with depth corrected for the $K_{\mathrm{o}}$ condition. Profile developed using normally consolidated triaxial test results. 


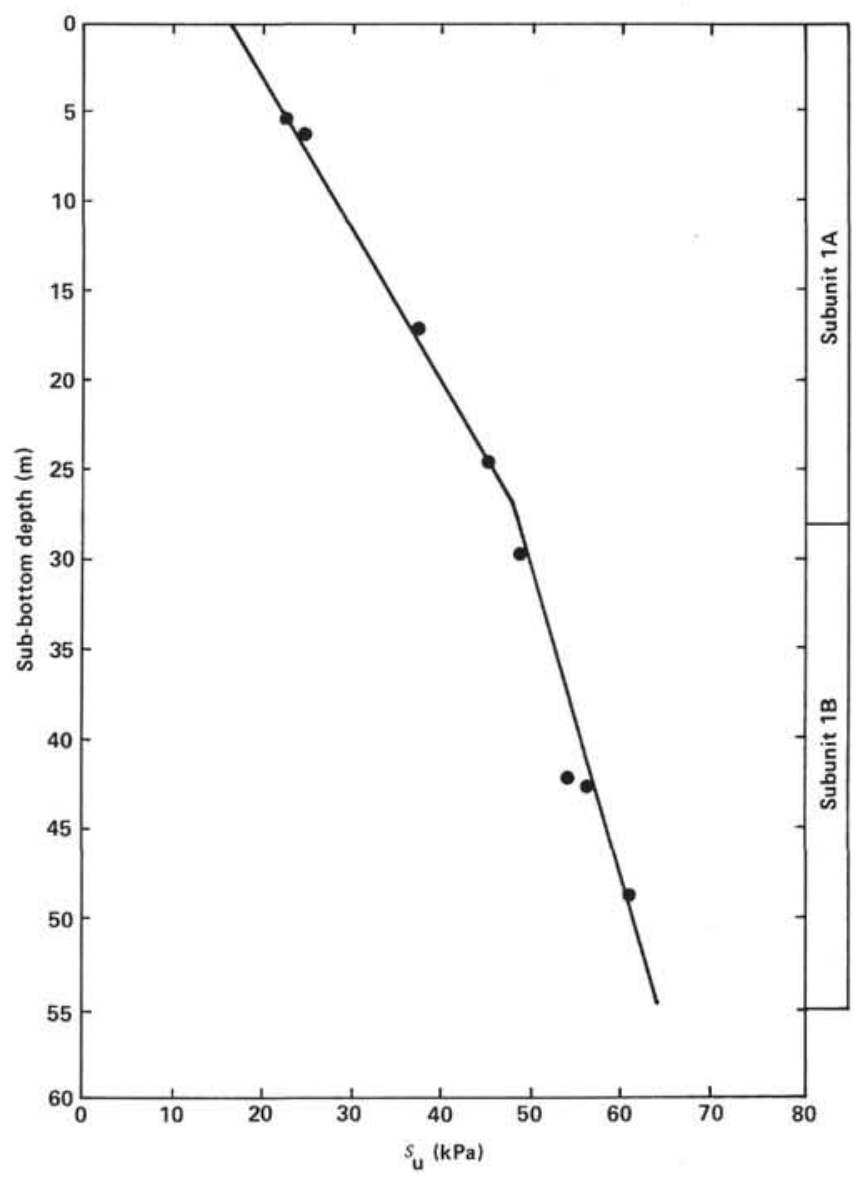

Figure 29. In situ shear strength-depth profile for Hole 576A sediments calculated using the SHANSEP technique. 


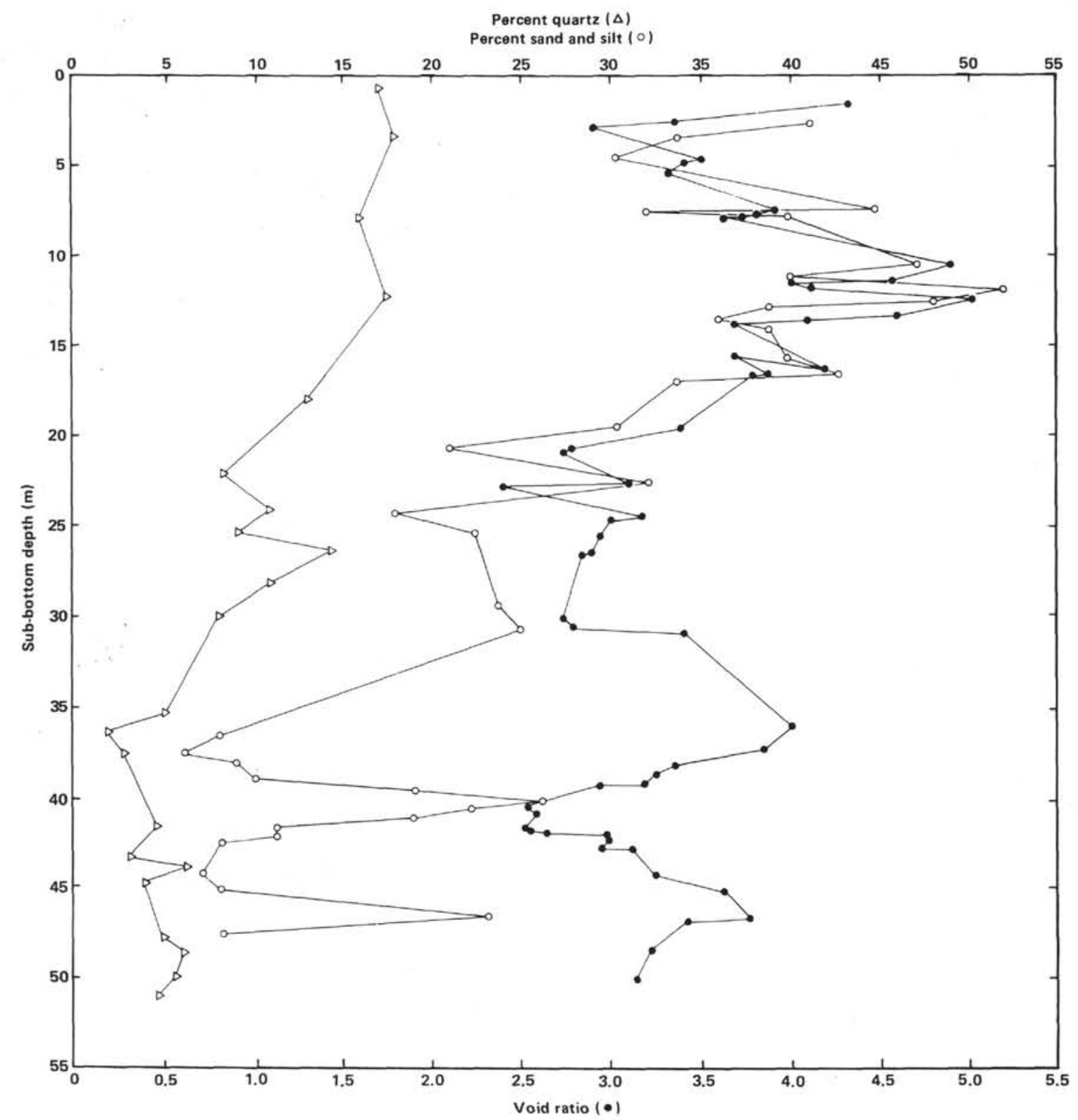

Figure 30. Variations in void ratio $(\bullet)$, sand- and silt-sized fraction $(0)$ and percent quartz $(\Delta)$ with depth in Hole 576A sediments. 


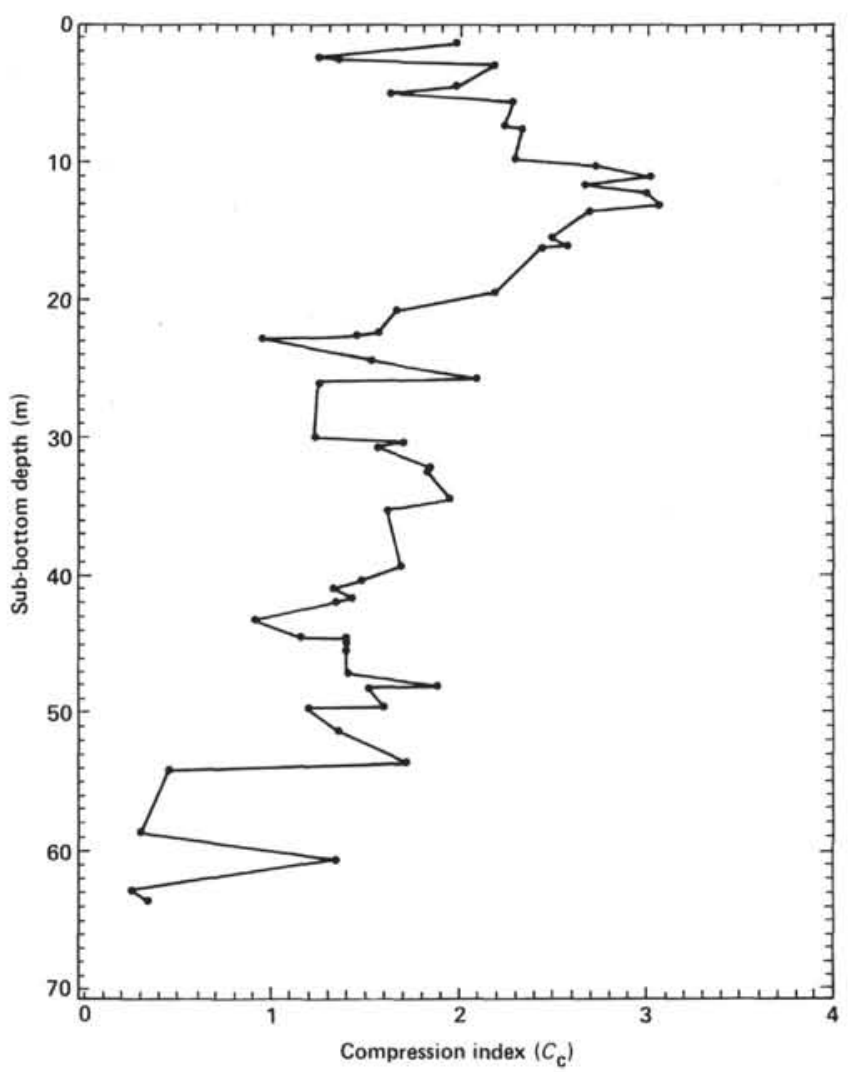

Figure 31. Compression index-depth profile determined using consolidation test results from Hole 576A sediments. 